\title{
Compactness criteria and new impulsive functional dynamic equations on time scales
}

\author{
Chao Wang $^{1 *}$ (D), Ravi P Agarwal ${ }^{2}$ and Donal O'Regan ${ }^{3}$
}

\section{"Correspondence:}

chaowang@ynu.edu.cn

'Department of Mathematics,

Yunnan University, Kunming,

Yunnan 650091, People's Republic of China

Full list of author information is available at the end of the article

\begin{abstract}
In this paper, we introduce the concept of $\Delta$-sub-derivative on time scales to define $\varepsilon$-equivalent impulsive functional dynamic equations on almost periodic time scales. To obtain the existence of solutions for this type of dynamic equation, we establish some new theorems to characterize the compact sets in regulated function space on noncompact intervals of time scales. Also, by introducing and studying a square bracket function $[x(\cdot), y(\cdot)]: \mathbb{T} \rightarrow \mathbb{R}$ on time scales, we establish some new sufficient conditions for the existence of almost periodic solutions for $\varepsilon$-equivalent impulsive functional dynamic equations on almost periodic time scales. The final section presents our conclusion and further discussion of this topic.
\end{abstract}

MSC: 34N05; 34A37; 39A24; 46B50

Keywords: relatively compact; existence; impulsive functional dynamic equations; almost periodic time scales

\section{Introduction}

The theory of calculus on time scales (see $[1,2]$ and references cited therein) was initiated by Stefan Hilger in 1988 (see [3]) to unify continuous and discrete analysis. In particular time the theory of scales unifies the study of differential and difference equations, and the qualitative analysis of dynamic equations on time scales is of particular importance (see [4-7]).

Time scales can be used to describe different natural phenomena in our real world and most changes in nature are inundated with periodic and almost periodic natural phenomena, so almost periodic problems of functional dynamic equations are important (see [813]) (typical examples are time intervals around a celestial body motion, the climate change during a year, the frequency of a tidal flood or an earthquake, etc.). We always study periodic or almost periodic problems assuming that the time scale $\mathbb{T}$ is periodic, that is, we always suppose the functions that describe the status of the object are periodic or almost periodic on periodic time scales (i.e., the status of the object is the same or almost the same after an accurately chosen interval; see [14] and its references). However, this is not always the case. Since the status of the object is frequently disturbed by its immediate state, the object's status is not always the same or almost the same after a precisely equal time interval. In fact frequently the object's status with periodicity or almost periodicity will be always the same or almost the same after 'an almost equivalent time interval', and we say the object has 'double almost periodicity'. To describe such a situation, recently, the

(c) 2016 Wang et al. This article is distributed under the terms of the Creative Commons Attribution 4.0 International License (http://creativecommons.org/licenses/by/4.0/), which permits unrestricted use, distribution, and reproduction in any medium, provided you give appropriate credit to the original author(s) and the source, provide a link to the Creative Commons license, and indicate if changes were made. 
authors introduced a type of time scales called 'almost periodic time scales' which can be adopted to accurately describe the status of the assigned object which is almost the same after an almost equivalent time interval (see [15-17]), and the authors studied the almost periodic dynamical behavior of impulsive delay dynamic models on almost periodic time scales.

Although the authors have this effective way to describe 'double almost periodicity', the models proposed in $[16,17])$ can be generalized with delays. In this paper, we investigate a new general type of impulsive functional dynamic equation and obtain some sufficient conditions for the existence of solutions. We now recall some ideas in [18] that will be used and improved in this paper. Using measure theory on time scales, the authors obtained some properties of almost periodic time scales, and they established a new class of delay dynamic equations which includes all almost periodic dynamic equations on periodic time scales if we assume that almost periodic time scales are equal to periodic time scales (see [18], Section 5). Let $\Pi_{\varepsilon}=E\{\mathbb{T}, \varepsilon\}, \mathbb{T}^{\Pi_{\varepsilon}}=\left\{\mathbb{T}^{-\tau}:-\tau \in E\{\mathbb{T}, \varepsilon\}\right\}$, where $E\{\mathbb{T}, \varepsilon\}$ is $\varepsilon$-translation number set of $\mathbb{T}$. Consider two types of delay dynamic equations.

Type $I$. For $t \in \mathbb{T} \cap\left(\cup_{-\tau} \mathbb{T}^{\Pi_{\varepsilon}}\right)$,

$$
x^{\Delta}(t)=g(t, x(t \pm \tau(t))), \quad \tau: \mathbb{T} \rightarrow \Pi_{\varepsilon} .
$$

Type II. For $t \in \mathbb{T} \cap\left(\cup_{-\tau} \mathbb{T}^{\Pi_{\varepsilon}}\right)$,

$$
x^{\Delta}(t)=g\left(t, \int_{a_{1}}^{b_{1}} x(t \pm \theta) \Delta_{\Pi_{\varepsilon}} \theta\right), \quad \theta \in\left[a_{1}, b_{1}\right]_{\Pi_{\varepsilon}} .
$$

Observe that if $\mathbb{T}$ is a periodic time scale, $\Pi_{\varepsilon}$ will turn into the periodicity set of $\mathbb{T}$, and then the dynamic equations (1.1) and (1.2) will include all delay dynamic equations in Section 4 from [18]. As a result the above two types of dynamic equations are more general than in the literature. Moreover, (1.1) and (1.2) are 'shaky slightly' since $\varepsilon$ is arbitrary, which is motivated by almost periodic time scales, and such a 'shake' occurs in the time variable. As a result this type of delay dynamic equation can describe the 'double periodicity' of the status of the object. Therefore, the existence of solutions for such a new type of dynamic equations with 'sight vibration' is significant not only for the theory of dynamic equations on time scales but also for practical applications.

Motivated by the above theoretical and practical significance, in this paper, we propose a general type of $\varepsilon$-equivalent impulsive functional dynamic equations with 'sight vibration' on almost periodic time scales as follows:

$$
\begin{cases}x^{\Delta}(t)=F\left(t, x(t), x_{t}\right), & t \neq t_{k}, t \in \mathbb{T} \cap\left(\cup \mathbb{T}^{\Pi_{\varepsilon}}\right), \\ \Delta x\left(t_{k}\right)=I_{k}\left(x\left(t_{k}\right)\right), & t=t_{k}, k \in \mathbb{Z},\end{cases}
$$

and obtain the existence of solutions (including almost periodic solutions) on almost periodic time scales.

This paper is organized as follows. In Section 2, we recall some necessary definitions of almost periodic time scales and introduce the concept of a $\Delta$-sub-derivative on time scales to define $\varepsilon$-equivalent impulsive functional dynamic equations on almost periodic time scales. In Section 3, we establish some new theorems to characterize the compact 
sets in regulated function space on noncompact intervals of time scales, which play an important role in establishing the existence of solutions for $\varepsilon$-equivalent impulsive functional dynamic equations with such a 'sight vibration'. In Section 4, we propose a type of function $[x(\cdot), y(\cdot)]$ (see Definition 4.1) on time scales and obtain some basic properties of it. Using this function $[x(\cdot), y(\cdot)]$, we establish some sufficient conditions for the existence of almost periodic solutions for such a class of $\varepsilon$-equivalent impulsive functional dynamic equations on almost periodic time scales. In Section 5, we present conclusions and further discussion of this topic.

\section{Preliminaries}

In this section, we first recall some basic definitions and lemmas, which will be used.

Let $\mathbb{T}$ be a nonempty closed subset (time scale) of $\mathbb{R}$. The forward and backward jump operators $\sigma, \rho: \mathbb{T} \rightarrow \mathbb{T}$ and the graininess $\mu: \mathbb{T} \rightarrow \mathbb{R}^{+}$are defined, respectively, by

$$
\sigma(t)=\inf \{s \in \mathbb{T}: s>t\}, \quad \rho(t)=\sup \{s \in \mathbb{T}: s<t\}, \quad \mu(t)=\sigma(t)-t .
$$

A point $t \in \mathbb{T}$ is called left-dense if $t>\inf \mathbb{T}$ and $\rho(t)=t$, left-scattered if $\rho(t)<t$, rightdense if $t<\sup \mathbb{T}$ and $\sigma(t)=t$, and right-scattered if $\sigma(t)>t$. If $\mathbb{T}$ has a left-scattered maximum $M$, then $\mathbb{T}^{k}=\mathbb{T} \backslash\{M\}$; otherwise $\mathbb{T}^{k}=\mathbb{T}$. If $\mathbb{T}$ has a right-scattered minimum $m$, then $\mathbb{T}_{k}=\mathbb{T} \backslash\{m\}$; otherwise $\mathbb{T}_{k}=\mathbb{T}$.

Definition 2.1 A function $f: \mathbb{T} \rightarrow \mathbb{R}$ is right-dense continuous provided it is continuous at right-dense point in $\mathbb{T}$ and its left-side limits exist at left-dense points in $\mathbb{T}$. If $f$ is continuous at each right-dense point and each left-dense point, then $f$ is said to be a continuous function on $\mathbb{T}$.

Definition 2.2 For $y: \mathbb{T} \rightarrow \mathbb{R}$ and $t \in \mathbb{T}^{k}$, we define the delta derivative of $y(t), y^{\Delta}(t)$, to be the number (if it exists) with the property that, for a given $\varepsilon>0$, there exists a neighborhood $U$ of $t$ such that

$$
\left|[y(\sigma(t))-y(s)]-y^{\Delta}(t)[\sigma(t)-s]\right|<\varepsilon|\sigma(t)-s|
$$

for all $s \in U$. Let $y$ be right-dense continuous, and if $Y^{\Delta}(t)=y(t)$, then we define the delta integral by

$$
\int_{a}^{t} y(s) \Delta s=Y(t)-Y(a)
$$

Definition 2.3 A function $p: \mathbb{T} \rightarrow \mathbb{R}$ is called regressive provided $1+\mu(t) p(t) \neq 0$ for all $t \in \mathbb{T}^{k}$. The set of all regressive and rd-continuous functions $p: \mathbb{T} \rightarrow \mathbb{R}$ will be denoted by $\mathcal{R}=\mathcal{R}(\mathbb{T})=\mathcal{R}(\mathbb{T}, \mathbb{R})$. We define the set $\mathcal{R}^{+}=\mathcal{R}^{+}(\mathbb{T}, \mathbb{R})=\{p \in \mathcal{R}: 1+\mu(t) p(t)>0, \forall t \in \mathbb{T}\}$.

An $n \times n$-matrix-valued function $A$ on a time scale $\mathbb{T}$ is called regressive provided

$$
I+\mu(t) A(t) \text { is invertible for all } t \in \mathbb{T},
$$

and the class of all such regressive and rd-continuous functions is denoted, similar to the above scalar case, by $\mathcal{R}=\mathcal{R}(\mathbb{T})=\mathcal{R}\left(\mathbb{T}, \mathbb{R}^{n \times n}\right)$. 
Definition 2.4 If $r$ is a regressive function, then the generalized exponential function $e_{r}$ is defined by

$$
e_{r}(t, s)=\exp \left\{\int_{s}^{t} \xi_{\mu(\tau)}(r(\tau)) \Delta \tau\right\}
$$

for all $s, t \in \mathbb{T}$, with the cylinder transformation

$$
\xi_{h}(z)= \begin{cases}\frac{\log (1+h z)}{h}, & \text { if } h \neq 0 \\ z, & \text { if } h=0\end{cases}
$$

For more details as regards dynamic equations on time scales, we refer the reader to [1-3].

In the following, we give some basic definitions and results of almost periodic time scales. For more details, one may consult [15-18].

Let $\tau$ be a number, and we set the time scales:

$$
\mathbb{T}:=\bigcup_{i=-\infty}^{+\infty}\left[\alpha_{i}, \beta_{i}\right], \quad \mathbb{T}^{\tau}:=\mathbb{T}+\tau=\{t+\tau: \forall t \in \mathbb{T}\}:=\bigcup_{i=-\infty}^{+\infty}\left[\alpha_{i}^{\tau}, \beta_{i}^{\tau}\right] .
$$

Define the distance between two time scales, $\mathbb{T}$ and $\mathbb{T}^{\tau}$ by

$$
d\left(\mathbb{T}, \mathbb{T}^{\tau}\right)=\max \left\{\sup _{i \in \mathbb{Z}}\left|\alpha_{i}-\alpha_{i}^{\tau}\right|, \sup _{i \in \mathbb{Z}}\left|\beta_{i}-\beta_{i}^{\tau}\right|\right\},
$$

where

$$
\alpha_{i}^{\tau}:=\inf \left\{\alpha \in \mathbb{T}^{\tau}:\left|\alpha_{i}-\alpha\right|\right\} \quad \text { and } \quad \beta_{i}^{\tau}:=\inf \left\{\beta \in \mathbb{T}^{\tau}:\left|\beta_{i}-\beta\right|\right\}
$$

Let

$$
\Pi:=\left\{\tau \in \mathbb{R}: \mathbb{T} \cap \mathbb{T}^{\tau} \neq \emptyset\right\} \neq\{0\}
$$

Next, for an arbitrary time scale $\mathbb{T}$, we can introduce the following new definition.

Definition 2.5 Let $S=\{\tilde{s} \in \mathbb{T}: \tilde{s}$ is a right- or left-scattered point in $\mathbb{T}\}$. For any $s \in \Pi$ and any right-scattered point $t$ in $\mathbb{T} \cap \mathbb{T}^{s}$, define $d_{t}=\inf _{\tilde{s} \in S}|t-\tilde{s}|$. Let the forward jump operator $\sigma_{s}(t)=\inf \left\{\hat{s} \in \mathbb{T} \cap \mathbb{T}^{s}: \hat{s}>t\right\}, t \in \mathbb{T} \cap \mathbb{T}^{s}$. We introduce a piecewise graininess function $\mu_{s}$ : $\mathbb{T} \cap \mathbb{T}^{s} \rightarrow \mathbb{R}^{+}$as follows:

$$
\mu_{s}(t)= \begin{cases}0, & t \text { is a right-dense point in } \mathbb{T} \cap \mathbb{T}^{s}, \\ \mu(t)+d_{\sigma(t)}, & t \text { is a right-scattered point in both } \mathbb{T} \cap \mathbb{T}^{s} \text { and } \mathbb{T}, \\ \mu\left(t+d_{t}\right), & \text { and } d_{t}=0, d_{\sigma(t)}>0, \\ & t \text { is a right-scattered point in } \mathbb{T} \cap \mathbb{T}^{s} \text { and a right-dense } \\ & \text { point in } \mathbb{T}, \text { and } d_{t}>0, d_{\sigma(t)}=0, \\ \mu\left(t+d_{t}\right)+d_{\sigma(t)}, & t \text { is a right-scattered point in } \mathbb{T} \cap \mathbb{T}^{s} \text { and a right-dense } \\ & \text { point in } \mathbb{T} \text { and } d_{t}, d_{\sigma(t)}>0, \\ & t \text { is a right-scattered point in both } \mathbb{T} \cap \mathbb{T}^{s} \text { and } \mathbb{T}, \\ & \text { and } d_{t}, d_{\sigma(t)}=0 .\end{cases}
$$




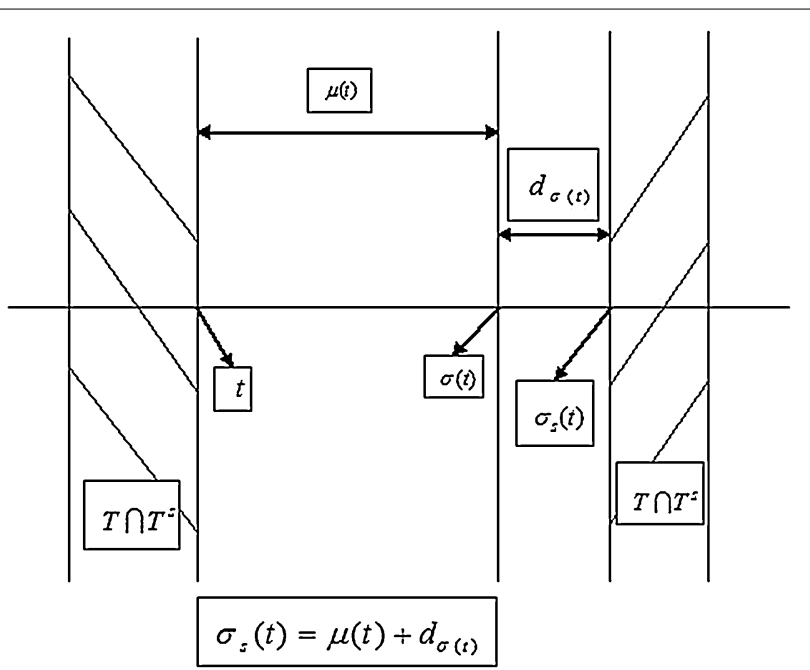

Figure $1 t$ is a right-scattered point in both $\mathbb{T} \cap \mathbb{T}^{s}$ and $\mathbb{T}$.

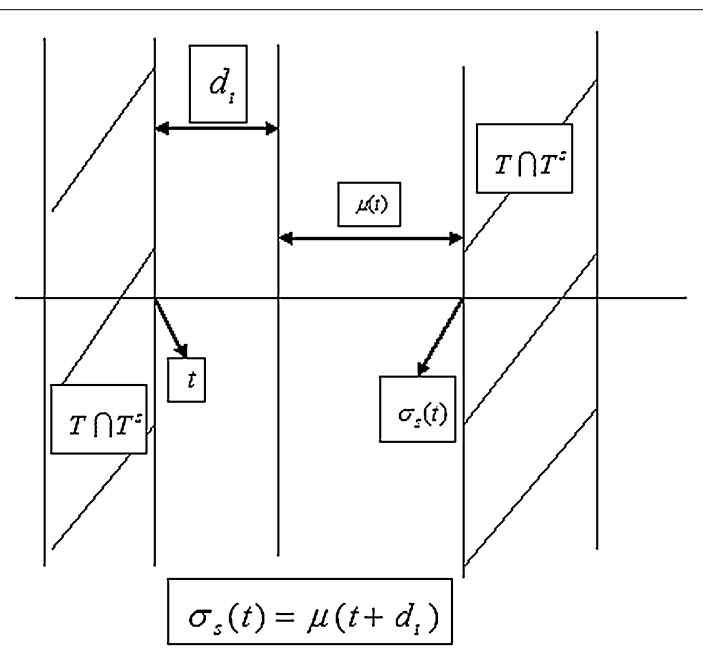

Figure $2 t$ is a right-scattered point in $\mathbb{T} \cap \mathbb{T}^{s}$ and a right-dense point in $\mathbb{T}$.

We say $\varphi: \mathbb{T} \rightarrow \mathbb{T}$ has a sub-derivative $\varphi^{\Delta_{s}}(\hat{t})$ on $\mathbb{T} \cap \mathbb{T}^{s}$ if

$$
\varphi^{\triangle_{s}}(\hat{t})=\lim _{t \rightarrow \hat{t}} \frac{\varphi\left(\sigma_{s}(t)\right)-\varphi(\hat{t})}{\sigma_{s}(t)-\hat{t}}
$$

exists for $t, \hat{t} \in \mathbb{T} \cap \mathbb{T}^{s}$ (see Figures 1-4).

In the following, we introduce some basic definitions of almost periodic time scales.

Definition 2.6 ([14, 16]) A subset $S$ of $\Pi$ is called relatively dense if there exists a positive number $L \in \Pi$ such that $[a, a+L]_{\Pi} \cap S \neq \emptyset$ for all $a \in \Pi$. The number $L$ is called the inclusion length. 


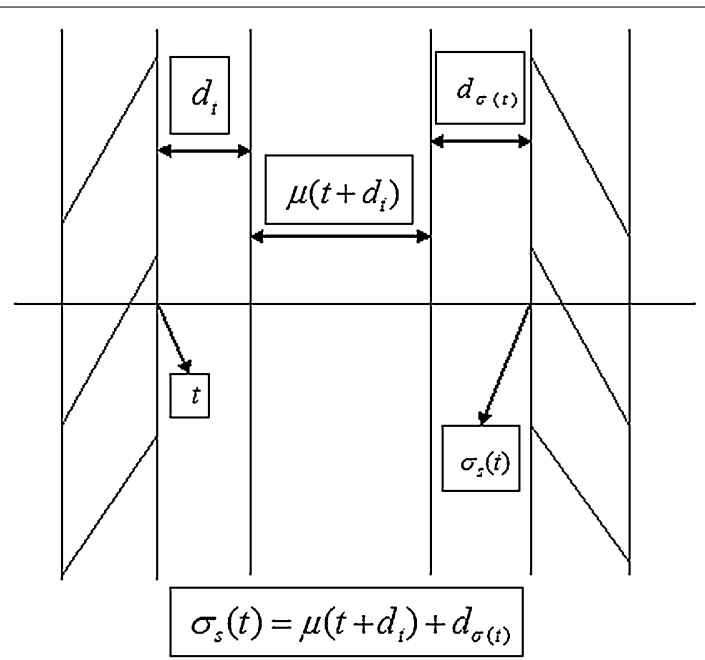

Figure $3 t$ is a right-scattered point in $\mathbb{T} \cap \mathbb{T}^{s}$ and a right-dense point in $\mathbb{T}$.

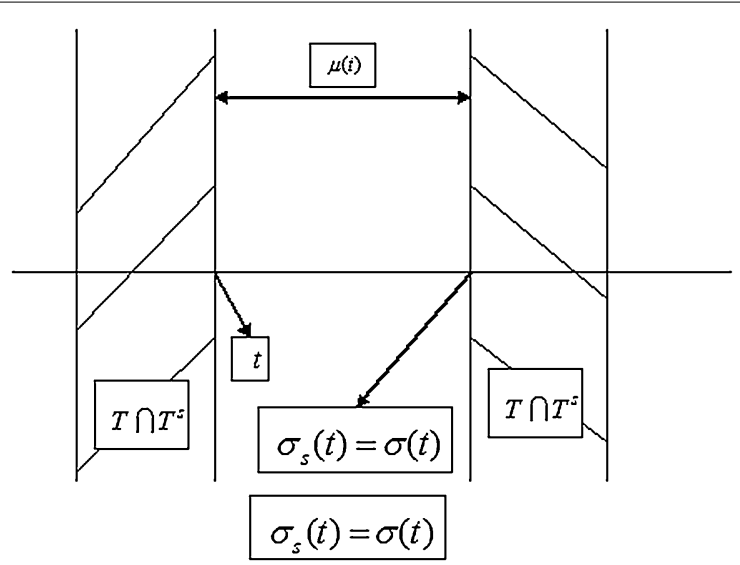

Figure $4 t$ is a right-scattered point in both $\mathbb{T} \cap \mathbb{T}^{s}$ and $\mathbb{T}$.

Then we can introduce the concept of almost periodic time scales as follows.

Definition $2.7([15,16])$ We say $\mathbb{T}$ is an almost periodic time scale if for any give $\varepsilon>0$, there exists a constant $l(\varepsilon)>0$ such that each interval of length $l(\varepsilon)$ contains a $\tau(\varepsilon) \in \Pi$ such that

$$
d\left(\mathbb{T}, \mathbb{T}^{\tau}\right)<\varepsilon
$$

i.e., for any $\varepsilon>0$, the set

$$
E\{\mathbb{T}, \varepsilon\}=\left\{\tau \in \Pi: d\left(\mathbb{T}^{\tau}, \mathbb{T}\right)<\varepsilon\right\}
$$

is relatively dense in $\Pi$. Now $\tau$ is called the $\varepsilon$-translation number of $\mathbb{T}$ and $l(\varepsilon)$ is called the inclusion length of $E\{\mathbb{T}, \varepsilon\}, E\{\mathbb{T}, \varepsilon\}$ is called the $\varepsilon$-translation set of $\mathbb{T}$, and for simplicity, we use the notation $E\{\mathbb{T}, \varepsilon\}:=\Pi_{\varepsilon}$. Note that $\sup \mathbb{T}=+\infty$,inf $\mathbb{T}=-\infty$. 
According to Definition 2.7, we obtain the following useful lemmas.

Lemma 2.1 Let $\mathbb{T}$ be an almost periodic time scale. If $\tau_{1}, \tau_{2} \in \Pi_{\varepsilon}$, then there exists $\xi_{0} \in$ $\left(\tau_{1}, \hat{\tau_{1}}+\tau_{2}\right]$ such that $\xi_{0} \in \Pi_{\varepsilon}$, where

$$
\left(\tau_{1}, \hat{\tau_{1}}+\tau_{2}\right]= \begin{cases}\left(\tau_{1}, \tau_{1}+\tau_{2}\right], & \tau_{1}<\tau_{1}+\tau_{2}, \\ {\left[\tau_{1}+\tau_{2}, \tau_{1}\right),} & \tau_{1}>\tau_{1}+\tau_{2} .\end{cases}
$$

Proof From the condition of the lemma, we obtain

$$
d\left(\mathbb{T}, \mathbb{T}^{\tau_{1}+\tau_{2}}\right)<d\left(\mathbb{T}, \mathbb{T}^{\tau_{1}}\right)+d\left(\mathbb{T}^{\tau_{1}}, \mathbb{T}^{\tau_{1}+\tau_{2}}\right)<2 \varepsilon
$$

Case $I$. If $d\left(\mathbb{T}, \mathbb{T}^{\tau_{1}+\tau_{2}}\right)<\varepsilon$, then $\xi_{0}=\tau_{1}+\tau_{2}$, and we get the desired result.

Case II. Let $2 \varepsilon>d\left(\mathbb{T}, \mathbb{T}^{\tau_{1}+\tau_{2}}\right)>\varepsilon$. Note (2.1), let

$$
f(x)=d\left(\mathbb{T}, \mathbb{T}^{x}\right), \quad x \in\left(\tau_{1}, \hat{\tau_{1}}+\tau_{2}\right]
$$

and note $f(x)$ is continuous on $\mathbb{R}$. Now, let $F(x)=f(x)-\varepsilon$, and we obtain

$$
F\left(\tau_{1}\right)=f\left(\tau_{1}\right)-\varepsilon<0, \quad F\left(\tau_{1}+\tau_{2}\right)=f\left(\tau_{1}+\tau_{2}\right)-\varepsilon>0,
$$

so, there exists some $\xi \in\left(\tau_{1}, \hat{\tau_{1}}+\tau_{2}\right)$ such that $F(\xi)=0$. From the continuity of $F$, we see that there exists some $\xi_{0} \in\left(\hat{\tau_{1}}, \xi\right)$ such that $F\left(\xi_{0}\right)<0$, i.e., $f\left(\xi_{0}\right)<\varepsilon$. This completes the proof.

Remark 2.1 From Lemma 2.1, we can see that $\Pi_{\varepsilon}$ is an infinite number set.

Lemma 2.2 For any $\varepsilon>0$ and $\tau_{1} \in \Pi_{\varepsilon}$, if $t_{0} \in \mathbb{T} \cap \mathbb{T}^{\tau_{1}}$, then there exists $\tau_{2} \in \Pi_{\varepsilon}$ with $\tau_{2}>\tau_{1}$ such that $t_{0} \in \mathbb{T} \cap \mathbb{T}^{\tau_{2}}$.

Proof From Lemma 2.1, there exists $\xi>\tau_{1}$ such that $d\left(\mathbb{T}, \mathbb{T}^{\xi}\right)<\varepsilon$.

Case $I$. If for $t_{0} \in \mathbb{T} \cap \mathbb{T}^{\tau_{1}}$, we have $t_{0} \in \mathbb{T} \cap \mathbb{T}^{\xi}$, and then $\tau_{2}=\xi \in \Pi_{\varepsilon}$.

Case II. If for $t_{0} \in \mathbb{T} \cap \mathbb{T}^{\tau_{1}}$, we have $t_{0} \notin \mathbb{T} \cap \mathbb{T}^{\xi}$, then $t_{0} \notin \mathbb{T}^{\xi}$ and

$$
d\left(\mathbb{T}^{\tau_{1}}, \mathbb{T}^{\xi}\right)<d\left(\mathbb{T}, \mathbb{T}^{\tau_{1}}\right)+d\left(\mathbb{T}, \mathbb{T}^{\xi}\right)<2 \varepsilon
$$

Then we denote $\gamma_{0}=\inf _{t \in \mathbb{T}^{\xi}}\left|t-t_{0}\right|+\varepsilon$, and we get

$$
\inf _{t \in \mathbb{T}^{\xi}}\left|t-t_{0}\right| \leq d\left(\mathbb{T}^{\xi}, \mathbb{T}\right)<\varepsilon
$$

so we have $\gamma_{0}<2 \varepsilon$. Therefore, by (2.3), we obtain

$$
\inf _{t \in \mathbb{T}^{\tau_{1}}}\left|\alpha_{i}^{\xi}+\gamma_{0}-t\right|<\varepsilon \quad \text { and } \quad \inf _{t \in \mathbb{T}^{\tau_{1}}}\left|\beta_{i}^{\xi}+\gamma_{0}-t\right|<\varepsilon \text {, }
$$

so

$$
\left|\alpha_{i}^{\xi}+\gamma_{0}-\alpha_{i}^{\tau_{1}}\right|<\varepsilon \text { and }\left|\beta_{i}^{\xi}+\gamma_{0}-\beta_{i}^{\tau_{1}}\right|<\varepsilon,
$$


and so we obtain

$$
\alpha_{i}^{\xi}+\gamma_{0}>\alpha_{i}^{\tau_{1}}-\varepsilon \text { and } \beta_{i}^{\xi}+\gamma_{0}>\beta_{i}^{\tau_{1}}-\varepsilon
$$

Hence,

$$
\begin{aligned}
d\left(\mathbb{T}, \mathbb{T}^{\xi+\gamma_{0}}\right) & =\max \left\{\sup _{i \in \mathbb{Z}}\left|\alpha_{i}-\left(\alpha_{i}^{\xi}+\gamma_{0}\right)\right|, \sup _{i \in \mathbb{Z}}\left|\beta_{i}-\left(\beta_{i}^{\xi}+\gamma_{0}\right)\right|\right\} \\
& \leq \max \left\{\sup _{i \in \mathbb{Z}}\left|\alpha_{i}-\left(\alpha_{i}^{\tau_{1}}-\varepsilon\right)\right|, \sup _{i \in \mathbb{Z}}\left|\beta_{i}-\left(\beta_{i}^{\tau_{1}}-\varepsilon\right)\right|\right\}=d\left(\mathbb{T}, \mathbb{T}^{\tau_{1}}\right)<\varepsilon .
\end{aligned}
$$

Hence, we have $\xi+\gamma_{0} \in \Pi_{\varepsilon}$ and $t_{0} \in \mathbb{T} \cap \mathbb{T}^{\xi+\gamma_{0}}$. Hence, we can take $\tau_{2}=\xi+\gamma_{0}>\tau_{1}$ such that $t_{0} \in \mathbb{T} \cap \mathbb{T}^{\tau_{2}}$. This completes the proof.

Remark 2.2 From Lemma 2.2, one see that if $\tau_{1} \in \Pi_{\varepsilon}$ and $t_{0} \in \mathbb{T} \cap \mathbb{T}^{\tau_{1}}$, then the set $\{\tau \in$ $\left.\Pi_{\varepsilon}: t_{0} \in \mathbb{T} \cap \mathbb{T}^{\tau}\right\}$ is an infinite number set.

Let $\mu_{\tau}: \mathbb{T}^{\tau} \rightarrow \mathbb{R}^{+}$be the graininess function of $\mathbb{T}^{\tau}$, and we obtain

$$
\mu_{\tau}(t+\tau)= \begin{cases}\mu(t), & t+\tau \notin \mathbb{T}, \\ \mu(t+\tau), & t+\tau \in \mathbb{T} .\end{cases}
$$

Thus, from (2.4), we can simplify Definition 2.7 as follows.

Definition 2.8 Let $\mu: \mathbb{T} \rightarrow \mathbb{R}^{+}$be a graininess function of $\mathbb{T}$. We say $\mathbb{T}$ is an almost periodic time scale if for any $\varepsilon>0$, the set

$$
\Pi^{*}=\left\{\tau \in \Pi:|\mu(t+\tau)-\mu(t)|<\varepsilon, \forall t \in \mathbb{T} \cap \mathbb{T}^{-\tau}\right\}
$$

is relatively dense in $\Pi$.

Definition 2.9 $([16,18])$ Let $\mathbb{T}$ be an almost periodic time scale, i.e., $\mathbb{T}$ satisfies Definition 2.7. A function $f \in C\left(\mathbb{T} \times D, \mathbb{E}^{n}\right)$ is called an almost periodic function in $t \in \mathbb{T}$ uniformly for $x \in D$ if the $\varepsilon_{2}$-translation set of $f$

$$
E\left\{\varepsilon_{2}, f, S\right\}=\left\{\tau \in \Pi_{\varepsilon_{1}}:|f(t+\tau, x)-f(t, x)|<\varepsilon_{2}, \text { for all }(t, x) \in\left(\mathbb{T} \cap \mathbb{T}^{-\tau}\right) \times S\right\}
$$

is a relatively dense set in $\Pi_{\varepsilon_{1}}$ for all $\varepsilon_{2}>\varepsilon_{1}>0$ and for each compact subset $S$ of $D$; that is, for any given $\varepsilon_{2}>\varepsilon_{1}>0$ and each compact subset $S$ of $D$, there exists a constant $l\left(\varepsilon_{2}, S\right)>0$ such that each interval of length $l\left(\varepsilon_{2}, S\right)$ contains a $\tau\left(\varepsilon_{2}, S\right) \in E\left\{\varepsilon_{2}, f, S\right\}$ such that

$$
|f(t+\tau, x)-f(t, x)|<\varepsilon_{2}, \quad \text { for all }(t, x) \in\left(\mathbb{T} \cap \mathbb{T}^{-\tau}\right) \times S .
$$

This $\tau$ is called the $\varepsilon_{2}$-translation number of $f$ and $l\left(\varepsilon_{2}, S\right)$ is called the inclusion length of $E\left\{\varepsilon_{2}, f, S\right\}$.

Remark 2.3 Note that Definition 5.5 from [18] is equivalent to Definition 2.9. For Definition 5.5 from [18], we note the following: 
(i) for all $t \in \mathbb{T} \cap\left(\cup \mathbb{T}^{\Pi_{\varepsilon_{1}}}\right)$, there exists $-\tau \in \Pi_{\varepsilon_{1}}$ such that $t \in \mathbb{T} \cap \mathbb{T}^{-\tau}$. From Remark 2.2, we know that the set $\left\{-\tau \in \Pi_{\varepsilon_{1}}: t \in \mathbb{T} \cap \mathbb{T}^{-\tau}\right\}$ is an infinite number set.

(ii) $d\left(\mathbb{T} \cap\left(\cup \mathbb{T}^{\Pi_{\varepsilon_{1}}}\right), \mathbb{T}\right)<\varepsilon_{1}$.

(iii) According to (i) from Remark 2.3, for all $t \in \mathbb{T} \cap\left(\cup \mathbb{T}^{\Pi_{\varepsilon_{1}}}\right)$, there exists an infinite number set $\bar{\Pi} \subset \Pi_{\varepsilon_{1}}$ such that $t+\tau \in \mathbb{T}$.

Definition $2.10([16,17])$ Let $\mathbb{T}$ be an almost periodic time scale and assume that $\left\{\tau_{i}\right\} \subset \mathbb{T}$ satisfying the derived sequence $\left\{\tau_{i}^{j}\right\}, i, j \in \mathbb{Z}$, is equipotentially almost periodic. We call a function $\varphi \in P C_{r d}\left(\mathbb{T}, \mathbb{R}^{n}\right)$ almost periodic if:

(i) for any $\varepsilon>0$, there is a positive number $\delta=\delta(\varepsilon)$ such that if the points $t^{\prime}$ and $t^{\prime \prime}$ belong to the same interval of continuity and $\left|t^{\prime}-t^{\prime \prime}\right|<\delta$, then $\left\|\varphi\left(t^{\prime}\right)-\varphi\left(t^{\prime \prime}\right)\right\|<\varepsilon$;

(ii) for any $\varepsilon_{2}>\varepsilon_{1}>0$, there is a relative dense set $\Gamma$ of $\varepsilon_{2}$-almost periods such that if $\tau \in \Gamma \subset \Pi_{\varepsilon_{1}}$, then $\|\varphi(t+\tau)-\varphi(t)\|<\varepsilon_{2}$ for all $t \in \mathbb{T} \cap\left(\cup \mathbb{T}^{\Pi_{\varepsilon_{1}}}\right)$ which satisfy the condition $\left|t-\tau_{i}\right|>\varepsilon_{2}, i \in \mathbb{Z}$.

Remark 2.4 From Definition 2.5, if $\mathbb{T}$ is a periodic time scale, then one can take a periodicity set $\tilde{\Pi} \subset \Pi$ of $\mathbb{T}$, such that $\mu_{s}(t)=\mu(t)$ for all $t \in \mathbb{T} \cap \mathbb{T}^{s}=\mathbb{T}, s \in \tilde{\Pi}, d_{t}=d_{\sigma(t)}=0$. If $\mathbb{T}$ is an almost periodic time scale from [15], then one can take a $\varepsilon$-translation number set $\Pi_{\varepsilon} \subset \Pi$ of $\mathbb{T}$, such that $\left|\mu_{s}(t)-\mu(t)\right|<\varepsilon$ for all $t \in \mathbb{T} \cap \mathbb{T}^{s}, s \in \Pi_{\varepsilon}$, i.e., for all right-scattered and right-dense points $t \in \mathbb{T} \cap \mathbb{T}^{s}$, one can obtain $d_{t}, d_{\sigma(t)}<\varepsilon$.

According to Definition 2.5 and Remark 2.4, we can introduce a concept of $\varepsilon$-equivalent impulsive functional dynamic equations on almost periodic time scales as follows.

Definition 2.11 Let $\mathbb{T}$ be an almost periodic time scale. Consider the following impulsive functional dynamic equations with sub-derivative $x^{\Delta_{-s}}(t)$ on $\mathbb{T} \cap \mathbb{T}^{-s}$ :

$$
\begin{cases}x^{\Delta_{-s}}(t)=f\left(t, x_{t}\right), & t \in \mathbb{T} \cap \mathbb{T}^{-s}, t \neq t_{k}, k \in \mathbb{Z}, \\ \Delta x\left(t_{k}\right)=I_{k}\left(x\left(t_{k}\right)\right), & t=t_{k}, k \in \mathbb{Z},\end{cases}
$$

where $-s \in \Pi, x_{t}(s)=x(t+s)$. We say the functional dynamic equations

$$
\begin{cases}x^{\Delta}(t)=f\left(t, x_{t}\right), & t \in \mathbb{T} \cap \mathbb{T}^{-s}, t \neq t_{k}, k \in \mathbb{Z}, \\ \Delta x\left(t_{k}\right)=I_{k}\left(x\left(t_{k}\right)\right), & t=t_{k}, k \in \mathbb{Z},\end{cases}
$$

are $\varepsilon$-equivalent impulsive functional dynamic equations for (2.5) if $-s \in \Pi_{\varepsilon} \subset \Pi$. Note that (2.6) can also be written as

$$
\begin{cases}x^{\Delta}(t)=f\left(t, x_{t}\right), & t \neq t_{k}, k \in \mathbb{Z}, \\ \Delta x\left(t_{k}\right)=I_{k}\left(x\left(t_{k}\right)\right), & t=t_{k}, k \in \mathbb{Z},\end{cases}
$$

where $t \in \mathbb{T} \cap\left(\cup \mathbb{T}^{\Pi_{\varepsilon}}\right)$. 
Remark 2.5 Let $\mathbb{T}$ be an almost periodic time scale. According to Theorem 5.2 and Remark 5.7 from [18], one can observe that we say the dynamic equations

$$
\begin{cases}x^{\Delta}(t)=f\left(t, x_{t}\right), & t \in \mathbb{T}, t \neq t_{k}, k \in \mathbb{Z}, \\ \Delta x\left(t_{k}\right)=I_{k}\left(x\left(t_{k}\right)\right), & t=t_{k}, k \in \mathbb{Z},\end{cases}
$$

have an almost periodic solution on the almost periodic time scale $\mathbb{T}$ if (2.7) has an almost periodic solution on $\mathbb{T} \cap \mathbb{T}^{-s}$ for any $-s \in \Pi_{\varepsilon}$.

Remark 2.6 According to Theorems 5.5 and 5.6 from [18], for (2.7), if we let $x_{t}(\tau(t))=$ $x(t-\tau(t)), \tau: \mathbb{T} \rightarrow \Pi_{\varepsilon}$, then it becomes

$$
\begin{cases}x^{\Delta}(t)=f(t, x(t-\tau(t))), & t \neq t_{k}, k \in \mathbb{Z}, \\ \Delta x\left(t_{k}\right)=I_{k}\left(x\left(t_{k}\right)\right), & t=t_{k}, k \in \mathbb{Z},\end{cases}
$$

if we let $x_{t}(\theta)=\int_{a}^{b} x(t+\theta) \Delta_{\Pi_{\varepsilon}} \theta$, and then it becomes

$$
\begin{cases}x^{\Delta}(t)=f\left(t, \int_{a}^{b} x(t+\theta) \Delta_{\Pi_{\varepsilon}} \theta\right), & t \neq t_{k}, k \in \mathbb{Z}, \\ \Delta x\left(t_{k}\right)=I_{k}\left(x\left(t_{k}\right)\right), & t=t_{k}, k \in \mathbb{Z} .\end{cases}
$$

Lemma 2.3 ([19]) Let A be a compact convex subset of a locally convex (linear topological) space and $f$ be a continuous map of $A$ into itself. Then $f$ has a fixed point.

\section{Characterizations of compact sets in a regulated functional space on time scales}

In this section, we introduce some new definitions and establish new characterization results of compact sets in functional spaces on time scales which will play an important role in studying abstract discontinuous dynamic equations on time scales.

First, let $\delta_{L}^{+\infty}, \delta_{R}^{+\infty}:\left[T_{0},+\infty\right)_{\mathbb{T}} \rightarrow \mathbb{R}^{+} \cup\{0\}$. Similar to [20], we can extend the $\Delta$-gauge for $[a, b]_{\mathbb{T}}$ to $\left[T_{0},+\infty\right)_{\mathbb{T}}$.

Definition 3.1 We say $\delta^{+\infty}=\left(\delta_{L}^{+\infty}, \delta_{R}^{+\infty}\right)$ is a $\Delta$-gauge for $\left[T_{0},+\infty\right)_{\mathbb{T}}$ provided $\delta_{L}^{+\infty}(t)>$ 0 on $\left(T_{0},+\infty\right)_{\mathbb{T}}$ and $\delta_{L}^{+\infty}\left(T_{0}\right) \geq 0, \delta_{R}^{+\infty}(t)>0$ on $\left[T_{0},+\infty\right)_{\mathbb{T}}$ and $\delta_{R}^{+\infty}(t) \geq \mu(t)$ for all $t \in$ $\left[T_{0},+\infty\right)_{\mathbb{T}}$.

For a $\Delta$-gauge, $\delta^{+\infty}$, we always assume $\delta_{L}^{+\infty}\left(T_{0}\right) \geq 0$ (we will sometimes not even point this out).

For $T_{0} \in \mathbb{T}$ and a Banach space $(X,\|\cdot\|)$, let

$$
\begin{aligned}
\mathfrak{G}\left(\left[T_{0},+\infty\right)_{\mathbb{T}}, X\right):= & \left\{x:\left[T_{0},+\infty\right)_{\mathbb{T}} \rightarrow X ;\right. \\
& \lim _{s \rightarrow t^{+}} x(s)=x\left(t^{+}\right) \text {and } \lim _{s \rightarrow t^{-}} x(s)=x\left(t^{-}\right) \text {exist and are finite, } \\
& \left.s, t<+\infty \text { and } \sup _{t \in\left[T_{0},+\infty\right)_{\mathbb{T}}}\|x(t)\|<+\infty\right\} .
\end{aligned}
$$

Endow $\mathfrak{G}\left(\left[T_{0},+\infty\right)_{\mathbb{T}}, X\right)$ with the norm $\|x\|_{\infty}=\sup _{t \in\left[T_{0},+\infty\right)_{\mathbb{T}}}\|x(t)\|$ 
Lemma $3.1\left(\mathfrak{G}\left(\left[T_{0},+\infty\right)_{\mathbb{T}}, X\right),\|\cdot\|_{\infty}\right)$ is a Banach space.

Proof Let $\left\{x_{n}\right\}$ be an arbitrary Cauchy sequence in $\mathfrak{G}$, i.e., for any $\varepsilon>0$, there exists $N$ such that $n, m>N$ implies

$$
\left\|x_{n}(t)-x_{m}(t)\right\|<\varepsilon \quad \text { for all } t \in\left[T_{0},+\infty\right)_{\mathbb{T}} .
$$

Since $X$ is a Banach space, for each $t \in\left[T_{0},+\infty\right)_{\mathbb{T}},\left\{x_{n}(t)\right\} \subset X$ is a Cauchy sequence so $x_{n}(t) \rightarrow x(t)$. Hence, let $m \rightarrow+\infty$ in (3.1), and we have

$$
\left\|x_{n}(t)-x(t)\right\|<\varepsilon \quad \text { for all } t \in\left[T_{0},+\infty\right)_{\mathbb{T}} .
$$

Furthermore, since $\left\{x_{n}\right\} \subset \mathfrak{G}$, for any $\varepsilon>0$ and $t_{0} \in\left[T_{0},+\infty\right)_{\mathbb{T}}$, there exists $\delta>0, t \in\left(t_{0}-\right.$ $\left.\delta_{L}^{+\infty}\left(t_{0}\right), t_{0}\right)_{\mathbb{T}}$ with

$$
\left\|x_{n}(t)-x_{n}\left(t_{0}^{+}\right)\right\|<\varepsilon
$$

Hence, we obtain

$$
\left\|x(t)-x\left(t_{0}^{+}\right)\right\| \leq\left\|x(t)-x_{n}(t)\right\|+\left\|x_{n}(t)-x_{n}\left(t_{0}^{+}\right)\right\|+\left\|x_{n}\left(t_{0}^{+}\right)-x\left(t_{0}^{+}\right)\right\| \leq 3 \varepsilon .
$$

Similarly, for $t \in\left(t_{0}, t_{0}+\delta_{R}^{+\infty}\left(t_{0}\right)\right)_{\mathbb{T}}$, we also obtain

$$
\left\|x(t)-x\left(t_{0}^{-}\right)\right\| \leq\left\|x\left(t_{0}\right)-x_{n}(t)\right\|+\left\|x_{n}(t)-x_{n}\left(t_{0}^{-}\right)\right\|+\left\|x_{n}\left(t_{0}^{-}\right)-x\left(t_{0}^{-}\right)\right\| \leq 3 \varepsilon .
$$

Therefore, from (3.2) and (3.3), we obtain $x \in \mathfrak{G}$. Hence, $\mathfrak{G}$ is a Banach space.

In the following, we will introduce the definition of a partition $\mathcal{P}$ for $\left[T_{0},+\infty\right)_{\mathbb{T}}$.

Definition 3.2 A partition $\mathcal{P}$ for $\left[T_{0},+\infty\right)_{\mathbb{T}}$ is a division of $\left[T_{0},+\infty\right)_{\mathbb{T}}$ denoted by

$$
\mathcal{P}=\left\{T_{0}=t_{0}^{\mathcal{P}} \leq \eta_{1} \leq t_{1}^{\mathcal{P}} \leq \cdots \leq t_{n-1}^{\mathcal{P}} \leq \eta_{n} \leq t_{n}^{\mathcal{P}} \leq \cdots<\cdots\right\}
$$

with $t_{i}^{\mathcal{P}}>t_{i-1}^{\mathcal{P}}$ for $i=1,2, \ldots$ and $t_{i}, \eta_{i} \in \mathbb{T}$. We call the points $\eta_{i}$ tag points and the points $t_{i}$ end points.

Definition 3.3 If $\delta^{+\infty}$ is a $\Delta$-gauge for $\left[T_{0},+\infty\right)_{\mathbb{T}}$, then we say a partition $\mathcal{P}$ is $\delta^{+\infty}$-fine if

$$
\eta_{i}-\delta_{L}^{+\infty}\left(\eta_{i}\right) \leq t_{i-1}^{\mathcal{P}}<t_{i}^{\mathcal{P}} \leq \eta_{i}+\delta_{R}^{+\infty}\left(\eta_{i}\right)
$$

for $i=1,2, \ldots$

Remark 3.1 From Definition 3.3, one can observe that if a partition $\mathcal{P}$ is $\delta^{+\infty}$-fine for $\left[T_{0},+\infty\right)_{\mathbb{T}}$, then for any closed interval $[a, b]_{\mathbb{T}} \subset\left[T_{0},+\infty\right)_{\mathbb{T}}$, there must exist a $\delta$-fine partition $\mathcal{P}^{*}$ and $\mathcal{P}^{*} \subset \mathcal{P}$. 
Definition 3.4 A set $\mathfrak{A} \subset \mathfrak{G}\left(\left[T_{0},+\infty\right)_{\mathbb{T}}, X\right)$ is called uniformly equi-regulated, if it has the following property: for every $\varepsilon>0$ and $t_{0} \in\left[T_{0},+\infty\right)_{\mathbb{T}}$, there is a $\delta^{+\infty}=\left(\delta_{L}^{+\infty}, \delta_{R}^{+\infty}\right)$ such that

(a) If $x \in \mathfrak{A}, t^{\prime} \in\left[T_{0},+\infty\right)_{\mathbb{T}}$ and $t_{0}-\delta_{L}^{+\infty}\left(t_{0}\right)<t^{\prime}<t_{0}$, then $\left\|x\left(t_{0}^{-}\right)-x\left(t^{\prime}\right)\right\|<\varepsilon$.

(b) If $x \in \mathfrak{A}, t^{\prime \prime} \in\left[T_{0},+\infty\right)_{\mathbb{T}}$ and $t_{0}<t^{\prime \prime}<t_{0}+\delta_{R}^{+\infty}\left(t_{0}\right)$, then $\left\|x\left(t_{0}^{+}\right)-x\left(t^{\prime \prime}\right)\right\|<\varepsilon$.

From Definition 3.4, we obtain the following theorem.

Theorem 3.1 A set $\mathfrak{A} \subset \mathfrak{G}\left(\left[T_{0},+\infty\right)_{\mathbb{T}}, X\right)$ is uniformly equi-regulated, if and only if, for every $\varepsilon>0$, there is a $\delta^{+\infty}$-fine partition $\mathcal{P}$ :

$$
T_{0}=t_{0}^{\mathcal{P}}<t_{1}^{\mathcal{P}}<t_{2}^{\mathcal{P}}<\cdots<t_{n}^{\mathcal{P}}<\cdots<\cdots
$$

such that

$$
\left\|x\left(t^{\prime}\right)-x(t)\right\| \leq \varepsilon
$$

for every $x \in \mathfrak{A}$ and $\left[t, t^{\prime}\right]_{\mathbb{T}} \subset\left(t_{j-1}^{\mathcal{P}}, t_{j}^{\mathcal{P}}\right)_{\mathbb{T}}, j=1,2, \ldots$

Proof Let $\varepsilon>0$ be given and let

$$
\mathfrak{D}=\left\{\xi ; \xi \in\left(T_{0},+\infty\right)_{\mathbb{T}}\right\}
$$

such that there is a partition $\mathcal{P}$ :

$$
T_{0}=t_{0}^{\mathcal{P}}<t_{1}^{\mathcal{P}}<\cdots<t_{k}^{\mathcal{P}}=\xi
$$

for which (3.4) holds with $j=1,2, \ldots, k$.

(i) If $\mathfrak{A} \subset \mathfrak{G}\left(\left[T_{0},+\infty\right)_{\mathbb{T}}, X\right)$ is uniformly equi-regulated, then there is a $\delta_{R}^{+\infty}\left(T_{0}\right)>0$ such that

$$
\left\|x(t)-x\left(T_{0}^{+}\right)\right\|<\frac{\varepsilon}{2}
$$

for every $x \in \mathfrak{A}$ and $t \in\left(T_{0}, T_{0}+\delta_{R}^{+\infty}\left(T_{0}\right)\right)_{\mathbb{T}}$. Denote $\xi_{1}=T_{0}+\delta_{R}^{+\infty}\left(T_{0}\right), T_{0}=t_{0}^{\mathcal{P}}<t_{1}^{\mathcal{P}}=\xi_{1}$. Thus, for $\left[t, t^{\prime}\right]_{\mathbb{T}} \subset\left(T_{0}, \xi_{1}\right)_{\mathbb{T}}$ and $x \in \mathfrak{A}$, the inequalities

$$
\left\|x(t)-x\left(t^{\prime}\right)\right\| \leq\left\|x(t)-x\left(T_{0}^{+}\right)\right\|+\left\|x\left(t^{\prime}\right)-x\left(T_{0}^{+}\right)\right\| \leq \varepsilon,
$$

holds and we have $\xi_{1} \in \mathfrak{D}$.

Let $\xi_{2}>\xi_{1}>T_{0}$. Since $x \in \mathfrak{A}$, then there is a $\delta_{L}^{+\infty}\left(\xi_{2}\right)$ such that

$$
\left\|x\left(\xi_{2}^{-}\right)-x(t)\right\|<\frac{\varepsilon}{2}
$$

for every $x \in \mathfrak{A}$ and $t \in\left(\xi_{2}-\delta_{L}^{+\infty}\left(\xi_{2}\right), \xi_{2}\right)_{\mathbb{T}} \cap\left[T_{0},+\infty\right)_{\mathbb{T}}$.

Let $\tilde{\xi} \in\left(\xi_{2}-\delta_{L}\left(\xi_{2}\right), \xi_{2}\right)_{\mathbb{T}}$ and a partition $T_{0}=t_{0}^{\mathcal{P}}<t_{1}^{\mathcal{P}}<t_{2}^{\mathcal{P}}<\cdots<t_{k}^{\mathcal{P}}=\tilde{\xi}$ be such that (3.4) holds with $j=1,2, \ldots, k$. Denote $t_{k+1}^{\mathcal{P}}=\xi_{2}$. Then for $\left[t, t^{\prime}\right]_{\mathbb{T}} \subset\left(t_{k}^{\mathcal{P}}, t_{k+1}^{\mathcal{P}}\right)_{\mathbb{T}}$ and $x \in \mathfrak{A}$, we have

$$
\left\|x(t)-x\left(t^{\prime}\right)\right\| \leq\left\|x(t)-x\left(\xi_{2}^{-}\right)\right\|+\left\|x\left(t^{\prime}\right)-x\left(\xi_{2}^{-}\right)\right\| \leq \varepsilon
$$


which implies $\xi_{2} \in \mathfrak{D}$. Thus we use the same argument as before to find that $\xi_{i} \in\left(\xi_{i-1},+\infty\right)_{\mathbb{T}}$ such that $\xi_{i} \in \mathfrak{D}, i=1,2, \ldots$. Hence $\xi_{\infty}=\sup \mathfrak{D}=+\infty$ and we are finished.

(ii) Reciprocally, for any given $\varepsilon>0$, there is a $\delta^{+\infty}$-fine partition $\mathcal{P}: T_{0}=t_{0}^{\mathcal{P}}<t_{1}^{\mathcal{P}}<t_{2}^{\mathcal{P}}<$ $\cdots<t_{k}^{\mathcal{P}}<\cdots<\cdots$ such that

$$
\left\|x(t)-x\left(t^{\prime}\right)\right\| \leq \varepsilon
$$

for every $x \in \mathfrak{A}$ and $\left[t, t^{\prime}\right]_{\mathbb{T}} \subset\left(t_{j-1}^{\mathcal{P}}, t_{j}^{\mathcal{P}}\right)_{\mathbb{T}}, j=1,2, \ldots$

Let $\eta_{j}$ be a tag of $\left(t_{j-1}, t_{j}\right)_{\mathbb{T}}$. Since this partition is $\delta^{+\infty}$-fine, we have $\left(t_{j-1}^{\mathcal{P}}, t_{j}^{\mathcal{P}}\right)_{\mathbb{T}} \subset\left(\eta_{j}-\right.$ $\left.\delta_{L}^{+\infty}\left(\eta_{j}\right), \eta_{j}+\delta_{R}^{+\infty}\left(\eta_{j}\right)\right)_{\mathbb{T}}$. Therefore, the inequality (3.5) holds, for $t, t^{\prime} \in\left(\eta_{j}-\delta_{L}^{+\infty}\left(\eta_{j}\right), \eta_{j}+\right.$ $\left.\delta_{R}^{+\infty}\left(\eta_{j}\right)\right)_{\mathbb{T}}$. Taking $t=\eta_{j}^{-}$and $t^{\prime} \in\left(\eta_{j}-\delta_{L}^{+\infty}\left(\eta_{j}\right), \eta_{j}\right]_{\mathbb{T}}$, then the inequality (3.5) remains true. Also, if $t=\eta_{j}^{+}$and $t^{\prime} \in\left[\eta_{j}, \eta_{j}+\delta_{R}^{+\infty}\left(\eta_{j}\right)\right)_{\mathbb{T}}$, the inequality (3.5) is fulfilled. Then, from Definition 3.4, it follows that $\mathfrak{A}$ is uniformly equi-regulated. This completes the proof.

Definition 3.5 Let $\mathfrak{A} \subset \mathfrak{G}\left(\left[T_{0},+\infty\right)_{\mathbb{T}}, X\right)$. We say $\mathfrak{A}$ is uniformly Cauchy if for any $\varepsilon>0$, there exist $T_{1} \in\left(T_{0},+\infty\right)_{\mathbb{T}}$ and a $\delta^{+\infty}=\left(\delta_{L}^{+\infty}, \delta_{R}^{+\infty}\right)$-fine partition $\mathcal{P}$ :

$$
t_{0}^{\mathcal{P}}=T_{1}<t_{1}^{\mathcal{P}}<t_{2}^{\mathcal{P}}<\cdots<t_{n}^{\mathcal{P}}<\cdots<\cdots
$$

such that:

(a) If $x \in \mathfrak{A}, t^{\prime}, t_{0} \in\left[T_{1},+\infty\right)_{\mathbb{T}}$ and $t_{0}-\delta_{L}^{+\infty}\left(t_{0}\right)<t^{\prime}<t_{0}$, then $\left\|x\left(t_{0}^{-}\right)-x\left(t^{\prime}\right)\right\|<\varepsilon$.

(b) If $x \in \mathfrak{A}, t^{\prime \prime}, t_{0} \in\left[T_{1},+\infty\right)_{\mathbb{T}}$ and $t_{0}<t^{\prime \prime}<t_{0}+\delta_{R}^{+\infty}\left(t_{0}\right)$, then $\left\|x\left(t_{0}^{+}\right)-x\left(t^{\prime \prime}\right)\right\|<\varepsilon$.

(c) If $x \in \mathfrak{A}, t^{\prime} \in\left[a^{\prime}, b^{\prime}\right]_{\mathbb{T}} \subset\left(t_{j-1}^{\mathcal{P}}, t_{j}^{\mathcal{P}}\right)_{\mathbb{T}}, t^{\prime \prime} \in\left[a^{\prime \prime}, b^{\prime \prime}\right]_{\mathbb{T}} \subset\left(t_{i-1}^{\mathcal{P}}, t_{i}^{\mathcal{P}}\right)_{\mathbb{T}}, i, j=1,2, \ldots$, then $\left\|x\left(t^{\prime}\right)-x\left(t^{\prime \prime}\right)\right\|<\varepsilon$.

Remark 3.2 From Definition 3.5, one can observe that if $\mathfrak{A} \subset \mathfrak{G}\left(\left[T_{0},+\infty\right)_{\mathbb{T}}, X\right)$ is uniformly Cauchy, then there exists $T_{1} \in\left(T_{0},+\infty\right)_{\mathbb{T}}$ such that $\mathfrak{A}$ is uniformly equi-regulated on $\left[T_{1},+\infty\right)_{\mathbb{T}}$.

Theorem 3.2 Assume that a set $\mathfrak{A} \subset \mathfrak{G}\left(\left[T_{0},+\infty\right)_{\mathbb{T}}, X\right)$ is uniformly equi-regulated and uniformly Cauchy, and for any $t \in\left[T_{0},+\infty\right)_{\mathbb{T}}$, there is a number $\beta_{t}$ such that, for $x \in \mathfrak{A}$,

$$
\left\|x(t)-x\left(t^{-}\right)\right\| \leq \beta_{t}, \quad\left\|x\left(t^{+}\right)-x(t)\right\| \leq \beta_{t}, \quad t \in\left[T_{0},+\infty\right)_{\mathbb{T}} .
$$

Then there is a constant $K>0$ such that $\left\|x(t)-x\left(T_{0}\right)\right\| \leq K$, for every $x \in \mathfrak{A}$ and $t \in$ $\left[T_{0},+\infty\right)_{\mathbb{T}}$.

Proof Since $\mathfrak{A}$ is uniformly Cauchy, according to Definition 3.5 , there exists $T_{1} \in\left(T_{0},+\infty\right)_{\mathbb{T}}$ such that

$$
\left\|x(t)-x\left(T_{1}\right)\right\|<1, \quad t \in\left[T_{1},+\infty\right)_{\mathbb{T}} .
$$

Let $\mathfrak{C}$ be the set of all $\tau \in\left(T_{0}, T_{1}\right]_{\mathbb{T}}$ such that there exists $K_{\tau}>0$ such that

$$
\left\|x(t)-x\left(T_{0}\right)\right\| \leq K_{\tau}
$$

for any $x \in \mathfrak{A}$ and $t \in\left[T_{0}, \tau\right]_{\mathbb{T}}$. 
Since $\mathfrak{A}$ is uniformly equi-regulated, there is a $\delta_{R}^{+\infty}\left(T_{0}\right)$ such that

$$
\left\|x(t)-x\left(T_{0}^{+}\right)\right\| \leq 1
$$

for every $x \in \mathfrak{A}$ and $t \in\left(T_{0}, T_{0}+\delta_{R}^{+\infty}\left(t_{0}\right)\right]_{\mathbb{T}}$. This fact together with the hypothesis implies that

$$
\left\|x(t)-x\left(T_{0}\right)\right\| \leq\left\|x(t)-x\left(T_{0}^{+}\right)\right\|+\left\|x\left(T_{0}^{+}\right)-x\left(T_{0}\right)\right\| \leq 1+\beta_{T_{0}}:=K_{T_{0}+\delta^{+}},
$$

for every $t \in\left(T_{0}, T_{0}+\delta_{R}\left(T_{0}\right)\right]_{\mathbb{T}}$ and $x \in \mathfrak{A}$. Hence, $\left(T_{0}, T_{0}+\delta_{R}\left(T_{0}\right)\right]_{\mathbb{T}} \subset \mathfrak{C}$.

Denote $\tau_{0}=\sup \mathfrak{C}$. As a consequence of the uniformly equi-regulatedness of $\mathfrak{A}$, there is a $\delta_{L}^{+\infty}\left(\tau_{0}\right)>0$ such that $\left\|x(t)-x\left(\tau_{0}^{-}\right)\right\| \leq 1$ for $x \in \mathfrak{A}$ and $t \in\left[\tau_{0}-\delta_{L}^{+\infty}\left(\tau_{0}\right), \tau_{0}\right)_{\mathbb{T}}$.

Let $\tau \in \mathfrak{C} \cap\left[\tau_{0}-\delta_{L}^{+\infty}\left(\tau_{0}\right), \tau_{0}\right)_{\mathbb{T}}$. Then

$$
\left\|x(t)-x\left(T_{0}\right)\right\| \leq\left\|x(t)-x\left(\tau_{0}^{-}\right)\right\|+\left\|x\left(\tau_{0}^{-}\right)-x(\tau)\right\|+\left\|x(\tau)-x\left(T_{0}\right)\right\| \leq 1+1+K_{\tau}=2+K_{\tau},
$$

for every $x \in \mathfrak{A}$ and $t \in\left(\tau, \tau_{0}\right)_{\mathbb{T}}$. Also,

$$
\left\|x\left(\tau_{0}^{-}\right)-x\left(T_{0}\right)\right\| \leq\left\|x\left(\tau_{0}^{-}\right)-x(\tau)\right\|+\left\|x(\tau)-x\left(T_{0}\right)\right\| \leq 1+K_{\tau} .
$$

These inequalities and this hypothesis imply that

$$
\left\|x\left(\tau_{0}\right)-x\left(T_{0}\right)\right\| \leq\left\|x\left(\tau_{0}\right)-x\left(\tau_{0}^{-}\right)\right\|+\left\|x\left(\tau_{0}^{-}\right)-x\left(T_{0}\right)\right\| \leq \beta_{\tau_{0}}+1+K_{\tau} .
$$

Thus $\tau_{0} \in \mathfrak{C}$, where $K_{\tau_{0}}=\beta_{\tau_{0}}+1+K_{\tau}$.

If $\tau_{0}<T_{1}$, then, since $\mathfrak{A}$ is uniformly equi-regulated, there is a $\delta_{R}^{+\infty}\left(\tau_{0}\right)>0$ such that

$$
\left\|x(t)-x\left(\tau_{0}^{+}\right)\right\| \leq 1, \quad \text { for any } x \in \mathfrak{A} \text { and } t \in\left(\tau_{0}, \tau_{0}+\delta_{R}\left(\tau_{0}\right)\right]_{\mathbb{T}},
$$

which implies

$$
\begin{aligned}
\left\|x(t)-x\left(T_{0}\right)\right\| & \leq\left\|x(t)-x\left(\tau_{0}^{+}\right)\right\|+\left\|x\left(\tau_{0}^{+}\right)-x\left(\tau_{0}\right)\right\|+\left\|x\left(\tau_{0}\right)-x\left(T_{0}\right)\right\| \\
& \leq 1+\beta_{\tau_{0}}+K_{\tau_{0}}=K_{\tau_{0}+\delta_{R}^{+\infty}\left(\tau_{0}\right)},
\end{aligned}
$$

for $t \in\left(\tau_{0}, \tau_{0}+\delta_{R}^{+\infty}\left(\tau_{0}\right)\right]_{\mathbb{T}}$ and $x \in \mathfrak{A}$. Thus $\tau_{0}+\delta_{R}^{+\infty}\left(\tau_{0}\right) \in \mathfrak{C}$, which contradicts the fact that $\tau_{0}=\sup \mathfrak{C}$. Therefore, $\tau_{0}=T_{1}$. Hence, by (3.8), we have

$$
\left\|x\left(T_{1}\right)-x\left(T_{0}\right)\right\| \leq K_{\tau_{0}} .
$$

Combining with (3.7), we have

$$
\left\|x(t)-x\left(T_{0}\right)\right\| \leq\left\|x(t)-x\left(T_{1}\right)\right\|+\left\|x\left(T_{0}\right)-x\left(T_{1}\right)\right\| \leq 1+K_{\tau_{0}},
$$

for $t \in\left(T_{1},+\infty\right)_{\mathbb{T}}$. Then we can get the desired result.

Now, we give some sufficient conditions to guarantee that $\mathfrak{A}$ is relatively compact in $\mathfrak{G}\left(\left[T_{0},+\infty\right)_{\mathbb{T}}, X\right)$. 
Theorem 3.3 Let $\mathfrak{A} \subset \mathfrak{G}\left(\left[T_{0},+\infty\right)_{\mathbb{T}}, X\right)$ be uniformly equi-regulated and uniformly Cauchy, for every $t \in\left[T_{0},+\infty\right)_{\mathbb{T}}$, and let the set $\{x(t) ; x \in \mathfrak{A}\}$ be relatively compact in $X$. Then the set $\mathfrak{A}$ is relatively compact in $\mathfrak{G}\left(\left[T_{0},+\infty\right)_{\mathbb{T}}, X\right)$.

Proof Since $\mathfrak{A}$ is uniformly Cauchy, $\forall x \in \mathfrak{A}$, by (a), (b) from Definition 3.5, for any $\varepsilon>0$, there exists $T_{1} \in\left(T_{0},+\infty\right)_{\mathbb{T}}$, there is a $\delta^{+\infty}=\left(\delta_{L}^{+\infty}, \delta_{R}^{+\infty}\right)$-fine partition $\mathcal{P}_{1}$ :

$$
t_{0}^{\mathcal{P}_{1}}=T_{1}<t_{1}^{\mathcal{P}_{1}}<t_{2}^{\mathcal{P}_{1}}<\cdots<t_{n}^{\mathcal{P}_{1}}<\cdots<\cdots
$$

such that

$$
\begin{aligned}
& \left\|x\left(t_{j}^{\mathcal{P}_{1}+}\right)-x\left(t^{\prime}\right)\right\|<\varepsilon \quad \text { for } t^{\prime} \in\left(t_{j}^{\mathcal{P}_{1}}, t_{j}^{\mathcal{P}_{1}}+\delta_{R}^{+\infty}\left(t_{j}^{\mathcal{P}_{1}}\right)\right]_{\mathbb{T}}, \\
& \left\|x\left(t_{j}^{\mathcal{P}_{1}-}\right)-x\left(t^{\prime \prime}\right)\right\|<\varepsilon \quad \text { for } t^{\prime \prime} \in\left[t_{j}^{\mathcal{P}_{1}}-\delta_{L}^{+\infty}\left(t_{j}^{\mathcal{P}_{1}}\right), t_{j}^{\mathcal{P}_{1}}\right)_{\mathbb{T}}, \\
& \left\|x\left(T_{1}^{+}\right)-x\left(t^{\prime \prime \prime}\right)\right\|<\varepsilon \quad \text { for } t^{\prime \prime \prime} \in\left(T_{1}, T_{1}+\delta_{R}^{+\infty}\left(T_{1}\right)\right]_{\mathbb{T}},
\end{aligned}
$$

for each $j=0,1,2, \ldots$. From (c) in Definition 3.5, we have

$$
\left\|x\left(T_{1}+\delta_{R}^{+\infty}\left(T_{1}\right)\right)-x(t)\right\|<\varepsilon \quad \text { for } t \in\left[t_{j-1}^{\mathcal{P}_{1}}+\delta_{R}^{+\infty}\left(t_{j-1}^{\mathcal{P}_{1}}\right), t_{j}^{\mathcal{P}_{1}}-\delta_{L}^{+\infty}\left(t_{j}^{\mathcal{P}_{1}}\right)\right]_{\mathbb{T}}
$$

and

$$
\left\|x\left(t^{\prime \prime \prime}\right)-x\left(t^{\prime}\right)\right\|<\varepsilon
$$

From (3.10) and (3.12), we have

$$
\begin{aligned}
\left\|x\left(T_{1}+\delta_{R}^{+\infty}\left(T_{1}\right)\right)-x\left(t_{j}^{\mathcal{P}_{1}-}\right)\right\| \leq & \left\|x\left(T_{1}+\delta_{R}^{+\infty}\left(T_{1}\right)\right)-x\left(t_{j}^{\mathcal{P}_{1}}-\delta_{L}^{+\infty}\left(t_{j}^{\mathcal{P}_{1}}\right)\right)\right\| \\
& +\left\|x\left(t_{j}^{\mathcal{P}_{1-}}\right)-x\left(t_{j}^{\mathcal{P}_{1}}-\delta_{L}^{+\infty}\left(t_{j}^{\mathcal{P}_{1}}\right)\right)\right\|<2 \varepsilon
\end{aligned}
$$

for each $j=0,1,2, \ldots$ Similarly, from (3.9), (3.11), and (3.13), we also have

$$
\begin{gathered}
\left\|x\left(T_{1}^{+}\right)-x\left(t_{j}^{\mathcal{P}_{1+}}\right)\right\| \leq \\
+\left\|x\left(T_{1}^{+}\right)-x\left(t^{\prime \prime \prime}\right)\right\|+\left\|x\left(t_{j}^{\mathcal{P}_{1+}}\right)-x\left(t^{\prime}\right)\right\| \\
+\left\|x\left(t^{\prime \prime \prime}\right)-x\left(t^{\prime}\right)\right\|<3 \varepsilon,
\end{gathered}
$$

for each $j=0,1,2, \ldots$ Hence, from (3.12), we obtain

$$
\left\|x\left(T_{1}+\delta_{R}^{+\infty}\left(T_{1}\right)\right)-x(t)\right\|<4 \varepsilon, \quad t \in\left[t_{j-1}^{\mathcal{P}_{1}}+\delta_{R}^{+\infty}\left(t_{j-1}^{\mathcal{P}_{1}}\right), t_{j}^{\mathcal{P}_{1}}-\delta_{L}^{+\infty}\left(t_{j}^{\mathcal{P}_{1}}\right)\right]_{\mathbb{T}} .
$$

From (3.10) and (3.14), we get

$$
\begin{aligned}
\left\|x\left(T_{1}+\delta_{R}^{+\infty}\left(T_{1}\right)\right)-x(t)\right\| & =\left\|x\left(t_{j}^{\mathcal{P}_{1}-}\right)-x(t)\right\|+\left\|x\left(T_{1}+\delta_{R}^{+\infty}\left(T_{1}\right)\right)-x\left(t_{j}^{\mathcal{P}_{1}-}\right)\right\| \\
& <4 \varepsilon, \quad t \in\left[t_{j}^{\mathcal{P}_{1}}-\delta_{L}^{+\infty}\left(t_{j}^{\mathcal{P}_{1}}\right), t_{j}^{\mathcal{P}_{1}}\right)_{\mathbb{T}^{\cdot}}
\end{aligned}
$$

Similarly, from (3.11) and (3.15), we also obtain

$$
\begin{aligned}
\left\|x\left(t_{j}^{\mathcal{P}_{1+}}\right)-x(t)\right\| & =\left\|x\left(T_{1}^{+}\right)-x(t)\right\|+\left\|x\left(T_{1}^{+}\right)-x\left(t_{j}^{\mathcal{P}_{1}+}\right)\right\| \\
& <4 \varepsilon, \quad t \in\left(t_{j}^{\mathcal{P}_{1}}, t_{j}^{\mathcal{P}_{1}}+\delta_{R}^{+\infty}\left(t_{j}^{\mathcal{P}_{1}}\right)\right]_{\mathbb{T}^{\prime}}
\end{aligned}
$$


so we obtain

$$
\left\|x\left(T_{1}^{+}\right)-x(t)\right\|<4 \varepsilon, \quad t \in\left(t_{j}^{\mathcal{P}_{1}}, t_{j}^{\mathcal{P}_{1}}+\delta_{R}^{+\infty}\left(t_{j}^{\mathcal{P}_{1}}\right)\right]_{\mathbb{T}} .
$$

Further, since $\mathfrak{A}$ is uniformly equi-regulated on $\left[T_{0},+\infty\right)_{\mathbb{T}}$, given $\varepsilon>0$, there is a $\delta^{+\infty}$ fine partition $\mathcal{P}_{2}$ :

$$
t_{0}^{\mathcal{P}_{2}}=T_{0}<t_{1}^{\mathcal{P}_{2}}<\cdots<t_{K}^{\mathcal{P}_{2}}=T_{1}
$$

such that

$$
\left\|x\left(t^{\prime}\right)-x(t)\right\|<\varepsilon
$$

for every $\left[t, t^{\prime}\right]_{\mathbb{T}} \subset\left(t_{j-1}^{\mathcal{P}_{2}}, t_{j}^{\mathcal{P}_{2}}\right)_{\mathbb{T}}, j \in\{1,2, \ldots, K\}$. Obviously, $\mathcal{P}=\mathcal{P}_{1} \cup \mathcal{P}_{2}$ is a $\delta^{+\infty}$-fine partition for $\left[T_{0},+\infty\right)_{\mathbb{T}}$.

Let $\left\{x_{n} ; n \in \mathbb{N}\right\}$ be a given sequence. By assumption, the set

$$
\left\{x_{n}\left(t_{j}^{\mathcal{P}_{2}}\right), x_{n}\left(T_{1}+\delta_{R}^{+\infty}\left(T_{1}\right)\right), x_{n}\left(T_{1}^{+}\right), n \in \mathbb{N}\right\}
$$

is relatively compact in $X$ for every $j=0,1,2, \ldots, K$. Therefore, we can find a subsequence of indices $\left\{n_{k} ; k \in \mathbb{N}\right\} \subset\{n ; n \in \mathbb{N}\}$ such that the set

$$
\left\{x_{n_{k}}\left(t_{j}^{\mathcal{P}_{2}}\right), x_{n_{k}}\left(T_{1}+\delta_{R}^{+\infty}\left(T_{1}\right)\right), x_{n_{k}}\left(T_{1}^{+}\right), k \in \mathbb{N}\right\}
$$

is also relatively compact in $X$ for every $j=0,1, \ldots, K$. Using this fact, we can find the elements $\left\{y_{j} ; j=0,1,2, \ldots, K, K+1, K+2\right\} \subset X$ such that $y_{j}=\lim _{k \rightarrow \infty} x_{n_{k}}\left(t_{j}^{\mathcal{P}_{2}}\right), y_{K+1}=$ $\lim _{k \rightarrow \infty} x_{n_{k}}\left(T_{1}+\delta_{R}^{+\infty}\left(T_{1}\right)\right)$ and $y_{K+2}=\lim _{k \rightarrow \infty} x_{n_{k}}\left(T_{1}^{+}\right)$. Therefore, there exists $N \in \mathbb{N}$ such that, for every $k>N$, we have

$$
\left\|x_{n_{k}}\left(t_{j}^{\mathcal{P}_{2}}\right)-y_{j}\right\|<\frac{\varepsilon}{4}
$$

Let $q>k$, and then

$$
\left\|x_{n_{q}}\left(t_{j}^{\mathcal{P}_{2}}\right)-y_{j}\right\|<\frac{\varepsilon}{4}
$$

for any $j=0,1, \ldots, K$. Similarly, we also have

$$
\begin{aligned}
& \left\|x_{n_{q}}\left(T_{1}+\delta_{R}^{+\infty}\left(T_{1}\right)\right)-y_{K+1}\right\|<\frac{\varepsilon}{4}, \\
& \left\|x_{n_{q}}\left(T_{1}^{+}\right)-y_{K+2}\right\|<\frac{\varepsilon}{4} .
\end{aligned}
$$

Now, let $t \in\left[T_{0}, T_{1}\right]_{\mathbb{T}}$ and $q \in \mathbb{N}$ such that $q>k$. Then $t=t_{j}^{\mathcal{P}_{2}}$ for some $j \in\{0,1, \ldots, K\}$ and, in this case, we have

$$
\left\|x_{n_{k}}(t)-x_{n_{q}}(t)\right\| \leq\left\|x_{n_{k}}\left(t_{j}^{\mathcal{P}_{2}}\right)-y_{j}\right\|+\left\|x_{n_{q}}\left(t_{j}^{\mathcal{P}_{2}}\right)-y_{j}\right\|<\frac{\varepsilon}{2}
$$


or $t \in\left(t_{j-1}^{\mathcal{P}_{2}}, t_{j}^{\mathcal{P}_{2}}\right)_{\mathbb{T}}$ for some $j \in\{1,2, \ldots, K\}$ and, in this case, we have

$$
\begin{aligned}
\left\|x_{n_{k}}(t)-x_{n_{q}}(t)\right\| & \leq\left\|x_{n_{k}}(t)-x_{n_{q}}\left(t_{j-1}^{\mathcal{P}_{2}}\right)\right\|+\left\|x_{n_{q}}(t)-x_{n_{k}}\left(t_{j-1}^{\mathcal{P}_{2}}\right)\right\|+\left\|x_{n_{q}}\left(t_{j-1}^{\mathcal{P}_{2}}\right)-x_{n_{k}}\left(t_{j-1}^{\mathcal{P}_{2}}\right)\right\| \\
& <\frac{\varepsilon}{4}+\frac{\varepsilon}{4}+\frac{\varepsilon}{2}=\varepsilon .
\end{aligned}
$$

Moreover, from (3.16), let $t \in\left[T_{1},+\infty\right)_{\mathbb{T}}$, we also obtain

$$
\begin{aligned}
\left\|x_{n_{k}}(t)-x_{n_{q}}(t)\right\| \leq & \left\|x_{n_{k}}(t)-x_{n_{q}}\left(T_{1}+\delta_{R}^{+\infty}\left(T_{1}\right)\right)\right\|+\left\|x_{n_{q}}(t)-x_{n_{k}}\left(T_{1}+\delta_{R}^{+\infty}\left(T_{1}\right)\right)\right\| \\
& +\left\|x_{n_{q}}\left(T_{1}+\delta_{R}^{+\infty}\left(T_{1}\right)\right)-x_{n_{k}}\left(T_{1}+\delta_{R}^{+\infty}\left(T_{1}\right)\right)\right\| \\
& <\frac{\varepsilon}{4}+\frac{\varepsilon}{4}+\frac{\varepsilon}{2}<\varepsilon
\end{aligned}
$$

for $t \in\left[t_{j-1}^{\mathcal{P}_{1}}+\delta_{R}^{+\infty}\left(t_{j-1}^{\mathcal{P}_{1}}\right), t_{j}^{\mathcal{P}_{1}}-\delta_{L}^{+\infty}\left(t_{j}^{\mathcal{P}_{1}}\right)\right]_{\mathbb{T}}, j \in\{1,2, \ldots\}$.

Similarly, from (3.17), we also obtain

$$
\begin{aligned}
\left\|x_{n_{k}}(t)-x_{n_{q}}(t)\right\| \leq & \left\|x_{n_{k}}(t)-x_{n_{q}}\left(T_{1}+\delta_{R}^{+\infty}\left(T_{1}\right)\right)\right\|+\left\|x_{n_{q}}(t)-x_{n_{k}}\left(T_{1}+\delta_{R}^{+\infty}\left(T_{1}\right)\right)\right\| \\
& +\left\|x_{n_{q}}\left(T_{1}+\delta_{R}^{+\infty}\left(T_{1}\right)\right)-x_{n_{k}}\left(T_{1}+\delta_{R}^{+\infty}\left(T_{1}\right)\right)\right\| \\
< & \frac{\varepsilon}{4}+\frac{\varepsilon}{4}+\frac{\varepsilon}{2}<\varepsilon,
\end{aligned}
$$

for $t \in\left[t_{j}^{\mathcal{P}_{1}}-\delta_{L}^{+\infty}\left(t_{j}^{\mathcal{P}_{1}}\right), t_{j}^{\mathcal{P}_{1}}\right)_{\mathbb{T}}, j \in\{0,1,2, \ldots\}$.

From (3.18), we have

$$
\begin{aligned}
\left\|x_{n_{k}}(t)-x_{n_{q}}(t)\right\| & \leq\left\|x_{n_{k}}(t)-x_{n_{q}}\left(T_{1}^{+}\right)\right\|+\left\|x_{n_{q}}(t)-x_{n_{k}}\left(T_{1}^{+}\right)\right\|+\left\|x_{n_{q}}\left(T_{1}^{+}\right)-x_{n_{k}}\left(T_{1}^{+}\right)\right\| \\
& <\frac{\varepsilon}{4}+\frac{\varepsilon}{4}+\frac{\varepsilon}{2}<\varepsilon,
\end{aligned}
$$

for $t \in\left(t_{j}^{\mathcal{P}_{1}}, t_{j}^{\mathcal{P}_{1}}+\delta_{R}^{+\infty}\left(t_{j}^{\mathcal{P}_{1}}\right)\right]_{\mathbb{T}}, j \in\{0,1,2, \ldots\}$.

Thus, for $t \in\left[T_{0},+\infty\right)_{\mathbb{T}}$, the sequence

$$
\left\{x_{n_{k}}(t) ; k \in \mathbb{N}\right\} \subset X
$$

is a Cauchy sequence. Since $X$ is complete, $\lim _{k \rightarrow \infty} x_{n_{k}}(t)$ exists. Hence, any $\left\{x_{n}\right\} \subset \mathfrak{A}$ has a convergent subsequence which means that $\mathfrak{A}$ is a relatively compact set. The proof is complete.

In the following, let $X=\mathbb{R}^{n}$; we will give some sufficient conditions to guarantee that $\mathfrak{A} \subset \mathfrak{G}\left(\left[T_{0},+\infty\right)_{\mathbb{T}}, \mathbb{R}^{n}\right)$ is relatively compact.

Theorem 3.4 Let a set $\mathfrak{A} \subset \mathfrak{G}\left(\left[T_{0},+\infty\right)_{\mathbb{T}}, \mathbb{R}^{n}\right)$. If $\mathfrak{A}$ is relatively compact in the sup-norm topology, then it is uniformly equi-regulated. If $\mathfrak{A}$ is uniformly equi-regulated and uniformly Cauchy, satisfying (3.6), then $\mathfrak{A}$ is relatively compact in $\mathfrak{G}\left(\left[T_{0},+\infty\right)_{\mathbb{T}}, \mathbb{R}^{n}\right)$.

Proof A subset $A$ of a Banach space $X$ is relatively compact if and only if it is totally bounded, i.e., for every $\varepsilon>0$, there is a finite $\varepsilon$-net $F$ for $A$, i.e., such a subset $F=$ $\left\{x_{1}, x_{2}, \ldots, x_{k}\right\}$ of $X$ that, for every $x \in A$, there is $x_{n} \in F$ satisfying $\left\|x-x_{n}\right\| \leq \varepsilon$. 
(i) Assume that $\mathfrak{A}$ is relatively compact. Then it is bounded by a constant $C$, and evidently (3.6) is satisfied with $\beta_{t}=2 C$ for every $t \in\left[T_{0},+\infty\right)_{\mathbb{T}}$.

Let $t_{0} \in\left[T_{0},+\infty\right)_{\mathbb{T}}$ and $\varepsilon$ be given. Let $\left\{x_{1}, x_{2}, \ldots, x_{k}\right\} \subset \mathfrak{G}\left(\left[T_{0},+\infty\right)_{\mathbb{T}}, \mathbb{R}^{n}\right)$ be a finite $\varepsilon / 3$ net for $\mathfrak{A}$. For every $n=1,2, \ldots, k$, there is a $\delta_{n}^{+\infty}=\left(\delta_{L}^{+\infty, n}, \delta_{R}^{+\infty, n}\right)$ such that

$$
\left|x_{n}(t)-x_{n}\left(t_{0}^{+}\right)\right|<\frac{\varepsilon}{3} \quad \text { for } t \in\left(t_{0}, t_{0}+\delta_{R}^{+\infty, n}\left(t_{0}\right)\right)_{\mathbb{T}} \cap\left[T_{0},+\infty\right)_{\mathbb{T}}
$$

and

$$
\left|x_{n}\left(t_{0}^{-}\right)-x_{n}(t)\right|<\frac{\varepsilon}{3} \quad \text { for } t \in\left(t_{0}-\delta_{L}^{+\infty, n}\left(t_{0}\right), t_{0}\right)_{\mathbb{T}} \cap\left[T_{0},+\infty\right)_{\mathbb{T}}
$$

Denote $\delta^{+\infty}=\left(\min _{1 \leq n \leq k}\left(\delta_{L}^{+\infty, n}\left(t_{0}\right)\right), \min _{1 \leq n \leq k}\left(\delta_{R}^{+\infty, n}\left(t_{0}\right)\right)\right)=\left(\delta_{L}^{+\infty}\left(t_{0}\right), \delta_{R}^{+\infty}\left(t_{0}\right)\right)$.

For arbitrary $x \in \mathfrak{A}$, we can find $x_{n}$ such that $\left\|x-x_{n}\right\|_{\infty} \leq \varepsilon / 3$ for every $t \in\left(t_{0}, t_{0}+\right.$ $\left.\delta_{R}^{+\infty}\left(t_{0}\right)\right)_{\mathbb{T}} \cap\left[T_{0},+\infty\right)_{\mathbb{T}}$, and we have the inequality

$$
\begin{aligned}
\left|x(t)-x\left(t_{0}^{+}\right)\right| & \leq\left|x(t)-x_{n}(t)\right|+\left|x_{n}(t)-x_{n}\left(t_{0}^{+}\right)\right|+\left|x_{n}\left(t_{0}^{+}\right)-x\left(t_{0}^{+}\right)\right| \\
& \leq 2\left\|x-x_{n}\right\|_{\infty}+\left|x_{n}(t)-x_{n}\left(t_{0}^{+}\right)\right|<\varepsilon,
\end{aligned}
$$

and similarly, $\left|x\left(t_{0}^{-}\right)-x(t)\right|<\varepsilon$ for $t \in\left(t_{0}-\delta_{L}^{+\infty}\left(t_{0}\right), t_{0}\right)_{\mathbb{T}}$.

(ii) Assume that $\mathfrak{A}$ is uniformly equi-regulated, (3.6) holds, i.e., there exists $\tilde{\alpha}>0$ such that $\left|x\left(T_{0}\right)\right| \leq \tilde{\alpha}$ for every $x \in \mathfrak{A}$.

From Theorem 3.2, there is $K$ such that $\left|x(t)-x\left(T_{0}\right)\right| \leq K$ for any $x \in \mathfrak{A}$ and $t \in$ $\left[T_{0},+\infty\right)_{\mathbb{T}}$. Hence, $|x(t)| \leq\left|x(t)+x\left(T_{0}\right)\right|+\left|x\left(T_{0}\right)\right| \leq K+\tilde{\alpha}$. If we denote $\tilde{\gamma}=K+\tilde{\alpha}$, then $\|x\| \leq \tilde{\gamma}$ for $x \in \mathfrak{A}$. Since $\mathfrak{A}$ is uniformly bounded in $\mathbb{R}^{n}, \mathfrak{A}$ is a sequentially compact closed set in $\mathbb{R}^{n}$, i.e., $\mathfrak{A}$ is relatively compact in $\mathbb{R}^{n}$. From Theorem 3.3 , one can obtain $\mathfrak{A}$ is a relatively compact set in $\mathfrak{G}\left(\left[T_{0},+\infty\right)_{\mathbb{T}}, \mathbb{R}^{n}\right)$. This completes the proof.

Remark 3.3 If $\tilde{\mathfrak{A}}$ is uniformly bounded, equi-continuous and uniformly Cauchy, then $\tilde{\mathfrak{A}} \subset$ $\mathfrak{A}$. Hence, one can observe that Lemma 4 from [21] is just a particular case of Theorem 3.4.

Remark 3.4 For $a, b \in \mathbb{T}$, let

$$
\begin{aligned}
\mathfrak{G}_{1}\left([a, b]_{\mathbb{T}}, X\right):= & \left\{x:[a, b]_{\mathbb{T}} \rightarrow X\right. \\
& \left.\lim _{s \rightarrow t^{+}} x(s)=x\left(t^{+}\right) \text {and } \lim _{s \rightarrow t^{-}} x(s)=x\left(t^{-}\right) \text {exist and are finite }\right\},
\end{aligned}
$$

and according to each $x \in \mathfrak{G}_{1}$, we can construct the set $\mathfrak{G}_{2}\left([a,+\infty)_{\mathbb{T}}, X\right)$, satisfying, for each $\tilde{x} \in \mathfrak{G}_{2}$ :

$$
\tilde{x}(t)= \begin{cases}x(t), & t \in[a, b]_{\mathbb{T}}, \\ x\left(b^{-}\right), & t \in(b,+\infty)_{\mathbb{T}} .\end{cases}
$$

One can immediately see that $\mathfrak{G}_{1}$ and $\mathfrak{G}_{2}$ are topological homeomorphic, i.e., there exists a homeomorphic mapping $f: \mathfrak{G}_{1} \rightarrow \mathfrak{G}_{2}$ such that $f(x)=\tilde{x}$ and $f^{-1}(\tilde{x})=x$. Since for any set $\mathfrak{A} \subset \mathfrak{G}_{1}$, according to the construction of the set $\mathfrak{G}_{2}$, one can see that $f(\mathfrak{A})$ is uniformly Cauchy. If the set $\{f(x) ; x \in \mathfrak{A}\}$ is relatively compact in $X$, then, by Theorem 3.3 , the relative 
compactness of $f(\mathfrak{A})$ is decided by the uniformly equi-regulatedness of $f(\mathfrak{A})$. However, for all $t \in(b,+\infty)_{\mathbb{T}}$, obviously, $f(\mathfrak{A})$ is uniformly equi-regulated. Thus the uniformly equiregulatedness of $f(\mathfrak{A})$ on $[a,+\infty)_{\mathbb{T}}$ is actually decided by the equi-regulatedness of $\mathfrak{A}$ on $[a, b]_{\mathbb{T}}$. Therefore, if $\mathfrak{A}$ is equi-regulated on $[a, b]_{\mathbb{T}}$, then $f(\mathfrak{A}) \subset \mathfrak{G}_{2}$ is relatively compact, i.e., $\mathfrak{A}$ is relatively compact in $\mathfrak{G}_{1}$.

From Theorem 3.4 and Remark 3.4, we can obtain the following corollaries.

Corollary 3.1 Let $X=\mathbb{R}^{n}$. A set $\mathfrak{A} \subset \mathfrak{G}_{1}$ is relatively compact if and only if it is equiregulated and for every $t \in[a, b]_{\mathbb{T}}$, the set $\{x(t) ; x \in \mathfrak{A}\}$ is bounded in $\mathbb{R}^{n}$.

Proof If $\mathfrak{A} \subset \mathfrak{G}_{1}$ is relatively compact, then $\mathfrak{A}$ is totally bounded in $\mathbb{R}^{n}$, and then for every $t \in[a, b]_{\mathbb{T}},\{x(t) ; x \in \mathfrak{A}\}$ is bounded in $\mathbb{R}^{n}$. Moreover, by Remark 3.4, there exists a homeomorphic mapping $f: \mathfrak{G}_{1} \rightarrow \mathfrak{G}_{2}$ such that $f(\mathfrak{A}) \subset \mathfrak{G}_{2}$ is relatively compact, which means that $f(\mathfrak{A}) \subset \mathfrak{G}_{2}$ is equi-regulated on $[a, b]_{\mathbb{T}}$ according to Theorem 3.4. Since $f$ is continuous and a one-to-one mapping, $\mathfrak{A}$ is equi-regulated on $[a, b]_{\mathbb{T}}$.

If $\mathfrak{A} \subset \mathfrak{G}_{1}$ is equi-regulated and for every $t \in[a, b]_{\mathbb{T}}$, the set $\{x(t) ; x \in \mathfrak{A}\}$ is bounded in $\mathbb{R}^{n}$, then $f(\mathfrak{A}) \subset \mathfrak{G}_{2}$ is equi-regulated and for every $t \in[a,+\infty)_{\mathbb{T}}$, the set $\{f(x) ; f(x) \in f(\mathfrak{A})\}$ is bounded in $\mathbb{R}^{n}$, according to Theorem $3.3, f(\mathfrak{A})$ is relatively compact in $\mathfrak{G}_{2}$, i.e., $\mathfrak{A}$ is relatively compact in $\mathfrak{G}_{1}$. This completes the proof.

Corollary 3.2 Let $\mathfrak{A} \subset \mathfrak{G}_{1}$ be equi-regulated, and for every $t \in[a, b]_{\mathbb{T}}$, let the set $\{x(t) ; x \in$ $\mathfrak{A}\}$ be relatively compact in $X$. Then the set $\mathfrak{A}$ is relatively compact in $\mathfrak{G}_{1}\left([a, b]_{\mathbb{T}}, X\right)$.

Proof From the assumption of this corollary and Remark 3.4, by Theorem 3.3, we can see that the set $f(\mathfrak{A})$ is relatively compact in $\mathfrak{G}_{2}\left([a,+\infty)_{\mathbb{T}}, X\right)$, i.e., the set $\mathfrak{A}$ is relatively compact in $\mathfrak{G}_{1}\left([a, b]_{\mathbb{T}}, X\right)$. This completes the proof.

Remark 3.5 In fact, if we let $\mathbb{T}=\mathbb{R}$, Corollaries 3.1 and 3.2 can include Corollary 2.4 from [22] and Theorem 1.13 from [23], respectively.

Similarly, for $\bar{T}_{0} \in \mathbb{T}$ and a Banach space $(X,\|\cdot\|)$, let

$$
\begin{aligned}
\mathfrak{G}\left(\left(-\infty, \bar{T}_{0}\right]_{\mathbb{T}}, X\right):= & \left\{x:\left(-\infty, \bar{T}_{0}\right]_{\mathbb{T}} \rightarrow X ;\right. \\
& \lim _{s \rightarrow t^{+}} x(s)=x\left(t^{+}\right) \text {and } \lim _{s \rightarrow t^{-}} x(s)=x\left(t^{-}\right) \text {exist and are finite, } \\
& \left.s, t<+\infty \text { and } \sup _{t \in\left(-\infty, \bar{T}_{0}\right]_{\mathbb{T}}}\|x(t)\|<+\infty\right\} .
\end{aligned}
$$

From Definition 3.1, we can also introduce a $\delta^{-\infty}$ and extend the $\Delta$-gauge for $[a, b]_{\mathbb{T}}$ to $\left(-\infty, \bar{T}_{0}\right]_{\mathbb{T}}$, and then we can repeat the same above discussion, and the following theorems can also be obtained (we omit the proofs).

Theorem 3.5 Assume that a set $\mathfrak{A} \subset \mathfrak{G}\left(\left(-\infty, \bar{T}_{0}\right]_{\mathbb{T}}, X\right)$ is uniformly equi-regulated and uniformly Cauchy, and for any $t \in\left(-\infty, \bar{T}_{0}\right]_{\mathbb{T}}$, there is a number $\beta_{t}$ such that, for $x \in \mathfrak{A}$,

$$
\left\|x(t)-x\left(t^{-}\right)\right\| \leq \beta_{t}, \quad\left\|x\left(t^{+}\right)-x(t)\right\| \leq \beta_{t}, \quad t \in\left(-\infty, \bar{T}_{0}\right]_{\mathbb{T}} .
$$


Then there is a constant $K>0$ such that $\left\|x(t)-x\left(\bar{T}_{0}\right)\right\| \leq K$, for every $x \in \mathfrak{A}$ and $t \in$ $\left(-\infty, \bar{T}_{0}\right]_{\mathbb{T}}$.

Theorem 3.6 Let $\mathfrak{A} \subset \mathfrak{G}\left(\left(-\infty, \bar{T}_{0}\right]_{\mathbb{T}}, X\right)$ be uniformly equi-regulated and uniformly Cauchy, for every $t \in\left(-\infty, \bar{T}_{0}\right]_{\mathbb{T}}$, let the set $\{x(t) ; x \in \mathfrak{A}\}$ be relatively compact in $X$. Then the set $\mathfrak{A}$ is relatively compact in $\mathfrak{G}\left(\left(-\infty, \bar{T}_{0}\right]_{\mathbb{T}}, X\right)$.

Theorem 3.7 Let a set $\mathfrak{A} \subset \mathfrak{G}\left(\left(-\infty, \bar{T}_{0}\right]_{\mathbb{T}}, \mathbb{R}^{n}\right)$. If $\mathfrak{A}$ is relatively compact in the sup-norm topology, then it is uniformly equi-regulated.If $\mathfrak{A}$ is uniformly equi-regulated and uniformly Cauchy, satisfying (3.19), then $\mathfrak{A}$ is relatively compact in $\mathfrak{G}\left(\left(-\infty, \bar{T}_{0}\right]_{\mathbb{T}}, \mathbb{R}^{n}\right)$.

For the more general case, for a Banach space $(X,\|\cdot\|)$, let

$$
\begin{aligned}
\mathfrak{G}\left((-\infty,+\infty)_{\mathbb{T}}, X\right):= & \left\{x:(-\infty,+\infty)_{\mathbb{T}} \rightarrow X ;\right. \\
& \lim _{s \rightarrow t^{+}} x(s)=x\left(t^{+}\right) \text {and } \lim _{s \rightarrow t^{-}} x(s)=x\left(t^{-}\right) \text {exist and are finite, } \\
& \left.-\infty<s, t<+\infty \text { and } \sup _{t \in(-\infty,+\infty)_{\mathbb{T}}}\|x(t)\|<+\infty\right\} .
\end{aligned}
$$

From Definition 3.1, we can also introduce a $\delta^{ \pm \infty}$ and extend the $\Delta$-gauge for $[a, b]_{\mathbb{T}}$ to $(-\infty,+\infty)_{\mathbb{T}}$, since for any $T_{0}>\bar{T}_{0},(-\infty,+\infty)_{\mathbb{T}}=\left(-\infty, \bar{T}_{0}\right]_{\mathbb{T}} \cup\left[\bar{T}_{0}, T_{0}\right]_{\mathbb{T}} \cup\left[T_{0},+\infty\right)_{\mathbb{T}}$, and then we can repeat the above discussion, and the above similar theorems can also be obtained (we omit these similar statements here).

Let

$$
\mathfrak{G}_{0}\left[T_{0},+\infty\right)_{\mathbb{T}}:=\left\{x ; x \in P C_{r d}\left(\left[T_{0},+\infty\right)_{\mathbb{T}}, \mathbb{R}^{n}\right) \text { and } \sup _{t \in\left[T_{0},+\infty\right)_{\mathbb{T}}}|x(t)|<+\infty\right\}
$$

where $P C_{r d}\left(\left[T_{0},+\infty\right)_{\mathbb{T}}, \mathbb{R}^{n}\right)$ is the set formed by all rd-piecewise continuous functions (one can consult Definition 2.4 from [24]). Endow $\mathfrak{G}_{0}$ with the norm $\|x\|=\sup _{t \in\left[T_{0},+\infty\right)_{\mathbb{T}}}|x(t)|$, and note $\left(\mathfrak{G}_{0},\|\cdot\|\right)$ is a Banach space.

Next, we will establish some theorems to guarantee that $\mathfrak{A} \subset \mathfrak{G}_{0}\left(\left[T_{0},+\infty\right)_{\mathbb{T}}, \mathbb{R}^{n}\right)$ is uniformly Cauchy and uniformly equi-regulated.

Now, we give a new definition called 'equi-absolutely continuity' for the function set $\mathfrak{A} \subset \mathfrak{G}_{0}$, which will be used in the following lemma's proof.

Definition 3.6 Let $\mathfrak{A} \subset \mathfrak{G}_{0}$. We say $\mathfrak{A}$ is equi-absolutely continuous if for any $f \in \mathfrak{A}$ and $\varepsilon>0$, there exists $\delta>0$, such that for any finite mutually disjoint open interval $\left(x_{i}, y_{i}\right)_{\mathbb{T}} \subset$ $\left[T_{0},+\infty\right)_{\mathbb{T}}(i=1,2, \ldots, n)$

$$
\sum_{i=1}^{n}\left(y_{i}-x_{i}\right)<\delta
$$

implies

$$
\sum_{i=1}^{n}\left|f\left(x_{i}\right)-f\left(y_{i}\right)\right|<\varepsilon .
$$


Lemma 3.2 Let $\mathfrak{A} \subset \mathfrak{G}_{0}\left(\left[T_{0},+\infty\right)_{\mathbb{T}}, \mathbb{R}^{n}\right)$ be uniformly bounded and

$$
\mathscr{Z}=\left\{t \in\left[T_{0},+\infty\right)_{\mathbb{T}}: x \text { is not } \Delta \text {-differentiable at } t\right\}
$$

and $\mu_{\Delta}(\mathscr{Z})=0$ for all $x \in \mathfrak{A}$, i.e., $x$ is $\Delta$-differentiable at $\left[T_{0},+\infty\right)_{\mathbb{T}} \backslash \mathscr{Z}$ and there exists $M>0$ such that $\left|x^{\Delta}(t)\right| \leq M$ for all $x \in \mathfrak{A}$. Then $\mathfrak{A}$ is uniformly equi-regulated, and for any $\varepsilon>0$, there exists $T_{1}>0$ such that, for any $x \in \mathfrak{A}$, the following is fulfilled:

$$
\left|\int_{\left[T_{0},+\infty\right)_{\mathbb{T}} \backslash \mathscr{Z}} x^{\Delta}(s) \Delta s\right| \leq \varepsilon
$$

Proof From the condition of the theorem, since $x$ is $\Delta$-differentiable at $\left[T_{0},+\infty\right)_{\mathbb{T}} \backslash \mathscr{Z}$ and there exists $M>0$ such that $\left|x^{\Delta}(t)\right| \leq M$, according to Corollary 1.68 from [1], we can obtain for all $t \in\left[T_{0},+\infty\right)_{\mathbb{T}} \backslash \mathscr{Z}, x(t)$ satisfies the Lipschitz condition

$$
\left|x\left(t_{1}\right)-x\left(t_{2}\right)\right| \leq M\left|t_{1}-t_{2}\right|, \quad \forall t_{1}, t_{2} \in\left[T_{0},+\infty\right)_{\mathbb{T}} \backslash \mathscr{Z} .
$$

So for each $N_{0} \in \mathbb{Z}^{+}$, we can obtain

$$
\sum_{j=1}^{N_{0}}\left|t_{j}^{1}-t_{j}^{2}\right|<\frac{\varepsilon}{M}, \quad\left(t_{j}^{1}, t_{j}^{2}\right)_{\mathbb{T}} \subset\left[T_{0},+\infty\right)_{\mathbb{T}},
$$

which implies

$$
\sum_{j=1}^{N_{0}}\left|x\left(t_{j}^{1}\right)-x\left(t_{j}^{2}\right)\right|<M \sum_{j=1}^{N_{0}}\left|t_{j}^{1}-t_{j}^{2}\right|<\varepsilon
$$

i.e., $\mathfrak{A}$ is equi-absolutely continuous on $\left[T_{0},+\infty\right)_{\mathbb{T}} \backslash \mathscr{Z}$. Hence, from (3.20) and (3.21), for all $t \in\left[T_{0},+\infty\right)_{\mathbb{T}} \backslash \mathscr{Z}, \mathfrak{A}$ is uniformly equi-regulated. For all $t_{0} \in \mathscr{Z}$, since $\mu_{\Delta}(\mathscr{Z})=0$, from (3.20) and (3.21), we can take $\delta^{+\infty}=\left(\delta_{L}^{+\infty}, \delta_{R}^{+\infty}\right)$ and $\delta_{L}, \delta_{R}<\frac{\varepsilon}{M}$, so

$$
\left|x\left(t_{0}^{+}\right)-x(t)\right|<\sum_{j=1}^{N_{0}}\left|x\left(t_{j}^{1}\right)-x\left(t_{j}^{2}\right)\right|<\varepsilon \quad \text { if } t \in\left(t_{0}, t_{0}+\delta_{R}^{+\infty}\left(t_{0}\right)\right)_{\mathbb{T}}
$$

and

$$
\left|x\left(t_{0}^{-}\right)-x(t)\right|<\sum_{j=1}^{N_{0}}\left|x\left(t_{j}^{1}\right)-x\left(t_{j}^{2}\right)\right|<\varepsilon \quad \text { if } t \in\left(t_{0}-\delta_{L}^{+\infty}\left(t_{0}\right), t_{0}\right)_{\mathbb{T}}
$$

and thus $\mathfrak{A}$ is uniformly equi-regulated on $\left[T_{0},+\infty\right)_{\mathbb{T}}$. According to Theorem 3.1 , for any closed interval $\left[a_{\mathcal{P}_{1}}, b_{\mathcal{P}_{1}}\right]_{\mathbb{T}}$, there is a $\delta_{1}^{+\infty}$-fine partition $\mathcal{P}_{1}$ :

$$
a_{\mathcal{P}_{1}}:=T_{0}=t_{0}^{\mathcal{P}_{1}}<t_{1}^{\mathcal{P}_{1}}<t_{2}^{\mathcal{P}_{1}}<\cdots<t_{N}^{\mathcal{P}_{1}}:=b_{\mathcal{P}_{1}}
$$

such that

$$
\left|x\left(t^{\prime}\right)-x\left(t^{\prime \prime}\right)\right| \leq \frac{1}{2},
$$


for every $x \in \mathfrak{A}$ and $\left[t^{\prime}, t^{\prime \prime}\right]_{\mathbb{T}} \subset\left(t_{j-1}^{\mathcal{P}_{1}}, t_{j}^{\mathcal{P}_{1}}\right)_{\mathbb{T}}, j=1,2, \ldots, N$. Hence, we have

$$
\left|x\left(t_{j}^{\mathcal{P}_{1}^{-}}\right)-x\left(t_{j-1}^{\mathcal{P}_{1}^{+}}\right)\right| \leq\left|x\left(t_{j}^{\mathcal{P}_{1}^{-}}\right)-x\left(t^{\prime}\right)\right|+\left|x\left(t^{\prime}\right)-x\left(t^{\prime \prime}\right)\right|+\left|x\left(t^{\prime \prime}\right)-x\left(t_{j-1}^{\mathcal{P}_{1}^{+}}\right)\right| \leq \frac{3}{2},
$$

where $t^{\prime} \in\left(t_{j}^{\mathcal{P}_{1}}-\delta_{L}^{+\infty}\left(t_{j}^{\mathcal{P}_{1}}\right), t_{j}^{\mathcal{P}_{1}}\right)_{\mathbb{T}}, t^{\prime \prime} \in\left(t_{j-1}^{\mathcal{P}_{1}}, t_{j-1}^{\mathcal{P}_{1}}+\delta_{R}^{+\infty}\left(t_{j-1}^{\mathcal{P}_{1}}\right)\right)_{\mathbb{T}}$.

Similarly, for any closed interval $\left[a_{\mathcal{P}_{2}}, b_{\mathcal{P}_{2}}\right]_{\mathbb{T}}$, there is a $\delta_{2}^{+\infty}$-fine partition $\mathcal{P}_{2}$ :

$$
a_{\mathcal{P}_{2}}:=b_{\mathcal{P}_{1}}=t_{0}^{\mathcal{P}_{2}}<t_{1}^{\mathcal{P}_{2}}<t_{2}^{\mathcal{P}_{2}}<\cdots<t_{N}^{\mathcal{P}_{2}}:=b_{\mathcal{P}_{2}}
$$

such that

$$
\left|x\left(t^{\prime}\right)-x\left(t^{\prime \prime}\right)\right| \leq \frac{1}{2^{2}}
$$

for every $x \in \mathfrak{A}$ and $\left[t^{\prime}, t^{\prime \prime}\right]_{\mathbb{T}} \subset\left(t_{j-1}^{\mathcal{P}_{2}}, t_{j}^{\mathcal{P}_{2}}\right)_{\mathbb{T}}, j=1,2, \ldots, N$. Hence, we have

$$
\left|x\left(t_{j}^{\mathcal{P}_{2}{ }^{-}}\right)-x\left(t_{j-1}^{\mathcal{P}^{+}}\right)\right| \leq\left|x\left(t_{j}^{\mathcal{P}^{-}}{ }^{-}\right)-x\left(t^{\prime}\right)\right|+\left|x\left(t^{\prime}\right)-x\left(t^{\prime \prime}\right)\right|+\left|x\left(t^{\prime \prime}\right)-x\left(t_{j-1}^{\mathcal{P}_{2}{ }^{+}}\right)\right| \leq \frac{3}{2^{2}},
$$

where $t^{\prime} \in\left(t_{j}^{\mathcal{P}_{2}}-\delta_{L}^{+\infty}\left(t_{j}^{\mathcal{P}_{2}}\right), t_{j}^{\mathcal{P}_{2}}\right)_{\mathbb{T}}, t^{\prime \prime} \in\left(t_{j-1}^{\mathcal{P}_{2}}, t_{j-1}^{\mathcal{P}_{2}}+\delta_{R}^{+\infty}\left(t_{j-1}^{\mathcal{P}_{2}}\right)\right)_{\mathbb{T}}$.

We can repeat the above process, then for each $i=2,3, \ldots$, we can see that, for any closed interval $\left[a_{\mathcal{P}_{i}}, b_{\mathcal{P}_{i}}\right]_{\mathbb{T}}$, there is a $\delta_{i}^{+\infty}$-fine partition $\mathcal{P}_{i}$ :

$$
a_{\mathcal{P}_{i}}:=b_{\mathcal{P}_{i-1}}=t_{0}^{\mathcal{P}_{i}}<t_{1}^{\mathcal{P}_{i}}<t_{2}^{\mathcal{P}_{i}}<\cdots<t_{N}^{\mathcal{P}_{i}}:=b_{\mathcal{P}_{i}}
$$

such that

$$
\left|x\left(t^{\prime}\right)-x\left(t^{\prime \prime}\right)\right| \leq \frac{1}{2^{i}}
$$

for every $x \in \mathfrak{A}$ and $\left[t^{\prime}, t^{\prime \prime}\right]_{\mathbb{T}} \subset\left(t_{j-1}^{\mathcal{P}_{i}}, t_{j}^{\mathcal{P}_{i}}\right)_{\mathbb{T}}, j=1,2, \ldots, N$. Hence, we have

$$
\left|x\left(t_{j}^{\mathcal{P}_{i}}{ }^{-}\right)-x\left(t_{j-1}^{\mathcal{P}_{i}^{+}}\right)\right| \leq\left|x\left(t_{j}^{\mathcal{P}_{i}^{-}}\right)-x\left(t^{\prime}\right)\right|+\left|x\left(t^{\prime}\right)-x\left(t^{\prime \prime}\right)\right|+\left|x\left(t^{\prime \prime}\right)-x\left(t_{j-1}^{\mathcal{P}_{i}{ }^{+}}\right)\right| \leq \frac{3}{2^{i}},
$$

where $t^{\prime} \in\left(t_{j}^{\mathcal{P}_{i}}-\delta_{L}^{+\infty}\left(t_{j}^{\mathcal{P}_{i}}\right), t_{j}^{\mathcal{P}_{i}}\right)_{\mathbb{T}}, t^{\prime \prime} \in\left(t_{j-1}^{\mathcal{P}_{i}}, t_{j-1}^{\mathcal{P}_{i}}+\delta_{R}^{+\infty}\left(t_{j-1}^{\mathcal{P}_{i}}\right)\right)_{\mathbb{T}}$. Thus, from (3.22), we obtain

$$
\sum_{i=1}^{+\infty} \sum_{j=1}^{N}\left|x\left(t_{j}^{\mathcal{P}_{i}{ }^{-}}\right)-x\left(t_{j-1}^{\mathcal{P}_{i}{ }^{+}}\right)\right| \leq 3 N \sum_{i=1}^{+\infty} \frac{1}{2^{i}}<+\infty,
$$

which implies that, for any $\varepsilon>0$, there exists $i_{0}>0$ such that

$$
\sum_{i=i_{0}}^{+\infty} \sum_{j=1}^{N}\left|x\left(t_{j}^{\mathcal{P}_{i}}{ }^{-}\right)-x\left(t_{j-1}^{\mathcal{P}_{i}^{+}}\right)\right| \leq \varepsilon
$$

which implies that, for any $\varepsilon>0$, there exists $T_{1} \geq t_{0}^{\mathcal{P}_{i_{0}}}$ and a partition

$$
\mathcal{P}:=\bigcup_{i=i_{0}}^{+\infty} \mathcal{P}_{i}
$$


i.e., $t_{0}^{\mathcal{P}_{i_{0}}}=t_{0}^{\mathcal{P}}<t_{1}^{\mathcal{P}_{i_{0}}}=t_{1}^{\mathcal{P}}<t_{2}^{\mathcal{P}_{i_{0}}}=t_{2}^{\mathcal{P}}<\cdots<t_{N}^{\mathcal{P}_{i_{0}}}=t_{N}^{\mathcal{P}}<t_{0}^{\mathcal{P}_{i_{0}+1}}=t_{N+1}^{\mathcal{P}}<t_{1}^{\mathcal{P}_{i_{0}+1}}=t_{N+2}^{\mathcal{P}}<\cdots<$ .. such that

$$
\sum_{j=1}^{+\infty}\left|x\left(t_{j}^{\mathcal{P}^{-}}\right)-x\left(t_{j-1}^{\mathcal{P}^{+}}\right)\right|=\sum_{i=i_{0}}^{+\infty} \sum_{j=1}^{N}\left|x\left(t_{j}^{\mathcal{P}_{i}^{-}}\right)-x\left(t_{j-1}^{\mathcal{P}_{i}^{+}}\right)\right| \leq \varepsilon
$$

Moreover, since $x$ is $\Delta$-differentiable almost everywhere on $\left[T_{0},+\infty\right)_{\mathbb{T}}$, we obtain

$$
\begin{aligned}
\left|\int_{\left[T_{1},+\infty\right)_{\mathbb{T}} \backslash \mathscr{Z}} x^{\Delta}(s) \Delta s\right| & =\sum_{j=1}^{+\infty}\left|\int_{\left(t_{j-1}^{\mathcal{P}}, t_{j}^{\mathcal{P}-}\right) \backslash \mathscr{Z}} x^{\Delta}(s) \Delta s\right| \\
& =\sum_{j=1}^{+\infty}\left|x\left(t_{j}^{\mathcal{P}^{-}}\right)-x\left(t_{j-1}^{\mathcal{P}^{+}}\right)\right| \leq \varepsilon .
\end{aligned}
$$

This completes the proof.

Theorem 3.8 Let $\mathfrak{A} \subset \mathfrak{G}_{0}\left(\left[T_{0},+\infty\right)_{\mathbb{T}}, \mathbb{R}^{n}\right)$ be uniformly bounded and

$$
\mathscr{Z}=\left\{t \in\left[T_{0},+\infty\right)_{\mathbb{T}}: x \text { is not } \Delta \text {-differentiable at } t\right\}
$$

and $\mu_{\Delta}(\mathscr{Z})=0$ for all $x \in \mathfrak{A}$, i.e., $x$ is $\Delta$-differentiable at $\left[T_{0},+\infty\right)_{\mathbb{T}} \backslash \mathscr{Z}$ and there exists $M>0$ such that $\left|x^{\Delta}(t)\right| \leq M$ for all $x \in \mathfrak{A}$. Then there exists $T_{1}>0$ such that $\mathfrak{A}$ is uniformly Cauchy on $\left[T_{1},+\infty\right)_{\mathbb{T}}$.

Proof According to Lemma 3.2, for any $\varepsilon>0$, there exists $T_{1}>0$ such that, for any $x \in \mathfrak{A}$, the following is fulfilled:

$$
\left|\int_{\left[T_{1},+\infty\right)_{\mathbb{T}} \backslash \mathscr{Z}} x^{\Delta}(s) \Delta s\right| \leq \varepsilon .
$$

Hence, for any $t_{1}, t_{2} \notin \mathscr{Z}, t_{1}, t_{2}>T_{1}$, we obtain

$$
\left|x\left(t_{1}\right)-x\left(t_{2}\right)\right|=\left|\int_{t_{1}}^{t_{2}} x^{\Delta}(s) \Delta s\right|<\left|\int_{\left[T_{1},+\infty\right)_{\mathbb{T}} \backslash \mathscr{Z}} x^{\Delta}(s) \Delta s\right| \leq \varepsilon .
$$

This completes the proof.

In the following, we will give the following useful corollaries.

Corollary 3.3 Let $\mathfrak{A} \subset \mathfrak{G}_{0}\left(\left[T_{0},+\infty\right)_{\mathbb{T}}, \mathbb{R}^{n}\right)$ be uniformly bounded and

$$
\mathscr{Z}=\left\{t \in\left[T_{0},+\infty\right)_{\mathbb{T}}: x \text { is not } \Delta \text {-differentiable at } t\right\}
$$

and $\mu_{\Delta}(\mathscr{Z})=0$ for all $x \in \mathfrak{A}$, and there exists $M>0$ such that $\left|x^{\Delta}(t)\right| \leq M$ for all $x \in \mathfrak{A}$. Then $\mathcal{A}$ is relatively compact in $\mathfrak{G}_{0}$.

Proof According to Theorem 3.8, $\mathfrak{A}$ is uniformly equi-regulated, uniformly Cauchy. Further, since $\mathfrak{A}$ is uniformly bounded, so it satisfies (3.6), by Theorem 3.4, we get the desired result immediately. 
Let

$$
\mathcal{B C}\left[T_{0},+\infty\right)_{\mathbb{T}}:=\left\{x \in B C\left(\left[T_{0},+\infty\right)_{\mathbb{T}}, \mathbb{R}^{n}\right) \text { and } \sup _{t \in\left[T_{0},+\infty\right)_{\mathbb{T}}}|x(t)|<\infty\right\}
$$

where $B C\left(\left[T_{0},+\infty\right)_{\mathbb{T}}, \mathbb{R}^{n}\right)$ denotes the set of all bounded continuous functions on $\left[T_{0}\right.$, $+\infty)_{\mathbb{T}}$. Then we can obtain the following corollary.

Corollary 3.4 Let $\mathfrak{A} \subset \mathcal{B C}\left[T_{0},+\infty\right)_{\mathbb{T}}$ be uniformly bounded and for all $x \in \mathfrak{A}, x$ is $\Delta$ differentiable and there exists $M>0$ such that $\left|x^{\Delta}(t)\right|<M$. Then $\mathfrak{A}$ is relatively compact in $\mathcal{B C}$.

Proof Since $x$ is $\Delta$-differentiable on $\left[T_{0},+\infty\right)_{\mathbb{T}}, \mathfrak{A}$ is equi-absolutely continuous on $\left[T_{0},+\infty\right)_{\mathbb{T}}$, for any $t_{1}, t_{2} \in\left[T_{0},+\infty\right)_{\mathbb{T}}$, we can obtain

$$
\left|x\left(t_{1}\right)-x\left(t_{2}\right)\right|=\left|\int_{t_{1}}^{t_{2}} x^{\Delta}(s) \Delta s\right| \leq 2 \sup _{t \in\left[T_{0},+\infty\right)_{\mathbb{T}}}|x(t)|<2 M_{0},
$$

which means that

$$
\left|\int_{t_{1}}^{+\infty} x^{\Delta}(s) \Delta s\right|<2 M_{0}, \quad M_{0} \text { is some constant. }
$$

Thus, for any $\varepsilon>0$, there exists $T_{1}>0$, and we have $t_{2}^{\prime}>t_{1}^{\prime}>T_{1}$ implies

$$
\left|x\left(t_{1}^{\prime}\right)-x\left(t_{2}^{\prime}\right)\right|=\left|\int_{t_{1}^{\prime}}^{t_{2}^{\prime}} x^{\Delta}(s) \Delta s\right|<\varepsilon,
$$

i.e., $\mathfrak{A}$ is uniformly Cauchy. According to Theorem 3.4, $\mathfrak{A}$ is relatively dense in $\mathcal{B C}$. This completes the proof.

Remark 3.6 Note that if for all $x \in \mathfrak{A} \subset \mathcal{B C}, x$ has uniformly bounded $\Delta$-derivatives, then one can see that $\mathfrak{A}$ is equi-absolutely continuous, which will lead to that $\mathfrak{A}$ is uniformly Cauchy. Hence, the uniformly boundedness of $\mathfrak{A}$ and the uniformly boundedness of $\Delta$ derivatives functions of $\mathfrak{A}$ can guarantee $\mathfrak{A}$ is relatively compact.

\section{Existence of solutions for impulsive functional dynamic equations}

In this section, we introduce some new definitions and give some new methods to obtain some sufficient conditions for the existence of solutions for a class of $\varepsilon$-equivalent impulsive functional dynamic equations on almost periodic time scales. We always assume that $\sup \mathbb{T}=+\infty$ and $\inf \mathbb{T}=-\infty$.

Definition 4.1 For arbitrary functions $x, y: \mathbb{T} \rightarrow \mathbb{R}^{n}$, we define the function $[x(\cdot), y(\cdot)]_{\mu}$ : $\mathbb{T} \rightarrow \mathbb{R}$ to be the number (provided it exists) with the property that, for any given $\varepsilon>0$, there exists a neighborhood $U$ of $t$ (i.e., $U=(t-\delta, t+\delta)_{\mathbb{T}}$ for some $\left.\delta>0\right)$ such that

$$
|| x(t)+|\sigma(t)-s| y(t)|-| x(t)|-| \sigma(t)-s\left|[x(\cdot), y(\cdot)]_{\mu}\right|<\varepsilon|\sigma(t)-s| \quad \text { for all } s \in U .
$$


Remark 4.1 In Definition 4.1, if $t$ is a right-dense point, $\mu(t)=0$, then one can obtain

$$
[x(t), y(t)]_{0}=\lim _{s \rightarrow t} \frac{|x(t)+| t-s|y(t)|-|x(t)|}{|t-s|},
$$

let $s-t=h \rightarrow 0^{+}$, and (4.1) can be written as

$$
[x(t), y(t)]_{0}=\lim _{h \rightarrow 0^{+}} \frac{|x(t)+h y(t)|-|x(t)|}{h} .
$$

If $t$ is a right-scattered point, $\mu(t)>0$, then one can get

$$
[x(t), y(t)]_{\mu}=\frac{|x(t)+\mu(t) y(t)|-|x(t)|}{\mu(t)} .
$$

In the following, we will consider the following impulsive system:

$$
\begin{cases}x^{\Delta}(t)=A(t, x)+g(t), & t \neq t_{k}, \\ \Delta x\left(t_{k}\right)=\tilde{I}_{k}\left(x\left(t_{k}\right)\right), & t=t_{k}, k \in \mathbb{Z},\end{cases}
$$

where $A \in P C_{r d}\left(\mathbb{T} \times \mathbb{R}^{n}, \mathbb{R}^{n}\right), g \in P C_{r d}\left(\mathbb{T}, \mathbb{R}^{n}\right), \tilde{I}_{k} \in C\left(\mathbb{R}^{n}, \mathbb{R}^{n}\right)$.

Next, we will give the following theorem to guarantee that (4.3) has a unique global solution.

Theorem 4.1 Let $\mathbb{T}$ be a time scale with the bounded graininess function $\mu$. In the system (4.3), iffor any $\varepsilon>0$, there exists a neighborhood $U$ of $t$ such that

$$
\tilde{d}\left(x(t)+|\sigma(t)-s|(A(t, x)+g(t)), B_{r}(0)\right)<\varepsilon|\sigma(t)-s|, \quad s \in U,
$$

for all $(t, x) \in\left(t_{k-1}, t_{k}\right)_{\mathbb{T}} \times B_{r}(0), k \in \mathbb{Z}$, where $\tilde{d}\left(z, B_{r}(0)\right)$ denotes the distance from $z$ to $B_{r}(0)$ and $B_{r}(0)=\left\{x \in P C_{r d}\left(\mathbb{T}, \mathbb{R}^{n}\right):\|x\| \leq r, r\right.$ is a constant $\}$. Then for each $t_{0}^{n} \in \mathbb{T}(n \in \mathbb{N})$, the Cauchy problem with the initial value $x\left(t_{0}^{n}\right)=u_{n}$ for (4.3) has a unique global solution $x(t)$ on $\mathbb{T}$ such that $x \in B_{r}(0)$ for all $t \in \mathbb{T}$.

Proof Consider the following form of (4.3):

$$
\begin{cases}x^{\Delta}(t)=\tilde{A} x(t)+A(t, x)-\tilde{A} x(t)+g(t), & t \neq t_{k}, \\ \Delta x\left(t_{k}\right)=\tilde{I}_{k}\left(x\left(t_{k}\right)\right), & t=t_{k}, k \in \mathbb{Z},\end{cases}
$$

where $\tilde{A}=A(t, \mathbf{0})$. From Theorem 2.77 from [1], for any $t_{0} \in \mathbb{T}$, we can find $k \in \mathbb{Z}, t_{k-1}<$ $t_{0} \leq t_{k}$, for $t \in\left[t_{0}, t_{k}\right)_{\mathbb{T}}$, there is a unique solution

$$
x(t)=e_{\tilde{A}}\left(t, t_{0}\right) x\left(t_{0}\right)+\int_{t_{0}}^{t} e_{\tilde{A}}(t, s)[A(s, x)-\tilde{A} x(s)+g(s)] \Delta s,
$$

and by using $x\left(t_{k}^{+}\right)-x\left(t_{k}^{-}\right)=\tilde{I}_{k}\left(x\left(t_{k}\right)\right)$, we obtain

$$
x\left(t_{k}^{+}\right)=e_{\tilde{A}}\left(t_{k}, t_{0}\right) x\left(t_{0}\right)+\int_{t_{0}}^{t_{k}} e_{\tilde{A}}\left(t_{k}, s\right)[A(s, x)-\tilde{A} x(s)+g(s)] \Delta s+\tilde{I}_{k}\left(x\left(t_{k}\right)\right),
$$


and then we have

$$
\begin{aligned}
x(t)= & e_{\tilde{A}}\left(t, t_{k}\right) x\left(t_{k}^{+}\right)+\int_{t_{k}}^{t} e_{\tilde{A}}(t, s)[A(s, x)-\tilde{A} x(s)+g(s)] \Delta s \\
= & e_{\tilde{A}}\left(t, t_{k}\right)\left[e_{\tilde{A}}\left(t_{k}, t_{0}\right) x\left(t_{0}\right)+\int_{t_{0}}^{t_{k}} e_{\tilde{A}}\left(t_{k}, s\right)[A(s, x)-\tilde{A} x(s)+g(s)] \Delta s+\tilde{I}_{k}\left(x\left(t_{k}\right)\right)\right] \\
& +\int_{t_{k}}^{t} e_{\tilde{A}}(t, s)[A(s, x)-\tilde{A} x(s)+g(s)] \Delta s \\
= & e_{\tilde{A}}\left(t, t_{0}\right) x\left(t_{0}\right)+\int_{t_{0}}^{t_{k}} e_{\tilde{A}}(t, s)[A(s, x)-\tilde{A} x(s)+g(s)] \Delta s+e_{\tilde{A}}\left(t, t_{k}\right) \tilde{I}_{k}\left(x\left(t_{k}\right)\right) \\
& +\int_{t_{k}}^{t} e_{\tilde{A}}(t, s)[A(s, x)-\tilde{A} x(s)+g(s)] \Delta s \\
= & e_{\tilde{A}}\left(t, t_{0}\right) x\left(t_{0}\right)+\int_{t_{0}}^{t} e_{\tilde{A}}(t, s)[A(s, x)-\tilde{A} x(s)+g(s)] \Delta s+e_{\tilde{A}}\left(t, t_{k}\right) \tilde{I}_{k}\left(x\left(t_{k}\right)\right) .
\end{aligned}
$$

Repeating this procedure, we get

$$
x(t)=e_{\tilde{A}}\left(t, t_{0}\right) x\left(t_{0}\right)+\int_{t_{0}}^{t} e_{\tilde{A}}(t, s)[A(s, x)-\tilde{A} x(s)+g(s)] \Delta s+\sum_{t_{0}<t_{k}<t} e_{\tilde{A}}\left(t, t_{k}\right) \tilde{I}_{k}\left(x\left(t_{k}\right)\right) .
$$

Hence, for $t \geq t_{0}$, we know that (4.5) has a unique solution satisfying

$$
\begin{aligned}
x\left(t, x_{0}\right)= & e_{\tilde{A}}\left(t, t_{0}\right) x_{0}+\int_{t_{0}}^{t} e_{\tilde{A}}(t, \sigma(s))(A(s, x)-\tilde{A} x(s)+g(s)) \Delta s \\
& +\sum_{t_{0}<t_{k}<t} e_{\tilde{A}}\left(t, t_{k}\right) \tilde{I}_{k}\left(x\left(t_{k}\right)\right) .
\end{aligned}
$$

Therefore, (4.3) has a unique global solution $u_{n}(t)$ on $\mathbb{T}$. Furthermore, combining with (4.3), for all $t \in\left(t_{k-1}, t_{k}\right)_{\mathbb{T}}, k \in \mathbb{Z}$, i.e., $t \neq t_{k}$, (4.4) can be changed into the following:

$$
\tilde{d}\left(x(t)+|\sigma(t)-s| x^{\Delta}(t), B_{r}(0)\right)<\varepsilon|\sigma(t)-s| .
$$

Case 1. If $t$ is a right-dense point, from (4.7), we obtain

$$
\tilde{d}\left(x(t)+h \lim _{h \rightarrow 0^{+}} \frac{x(t+h)-x(t)}{h}, B_{r}(0)\right)<\varepsilon h,
$$

that is,

$$
\tilde{d}\left(x(t), B_{r}(0)\right)<\varepsilon .
$$

Case 2. If $t$ is a right-scattered point, from (4.7), we get

$$
\tilde{d}\left(x(t)+\mu(t) \cdot \frac{x(\sigma(t))-x(t)}{\mu(t)}, B_{r}(0)\right)<\varepsilon \mu(t) \leq \varepsilon \bar{\mu}, \quad \bar{\mu}=\sup _{t \in \mathbb{T}} \mu(t),
$$

that is,

$$
\tilde{d}\left(x(\sigma(t)), B_{r}(0)\right)<\varepsilon .
$$


Thus, if $\rho(t)<t$, that is, $\rho(t)$ is a right-scattered point, we have $\sigma(\rho(t))=t$, by (4.9), one has

$$
\tilde{d}\left(x(t), B_{r}(0)\right)=\tilde{d}\left(x(\sigma(\rho(t))), B_{r}(0)\right)<\varepsilon .
$$

If $\rho(t)=t$, that is, $t$ is a left-dense point, so there must exist a right-dense point $s$, for any $\varepsilon>0$, there exists a $\delta_{L}^{+\infty}>0$ such that $s \in\left(t-\delta_{L}^{+\infty}(t), t\right)_{\mathbb{T}}$ implies

$$
\tilde{d}(x(s), x(t))<\varepsilon .
$$

Hence, by (4.8), we can easily get

$$
\tilde{d}\left(x(t), B_{r}(0)\right)<\tilde{d}(x(t), x(s))+\tilde{d}\left(x(s), B_{r}(0)\right)<\varepsilon+\varepsilon=2 \varepsilon, \quad t \neq t_{k}, k \in \mathbb{Z} .
$$

For the impulsive points $t_{k}, k \in \mathbb{Z}$, since $\left(t_{k}-\delta_{L}^{+\infty}\left(t_{k}\right), t_{k}\right)_{\mathbb{T}} \subset\left(t-\delta_{L}^{+\infty}(t), t+\delta_{R}^{+\infty}(t)\right)_{\mathbb{T}}$, where $\left|t-t_{k}\right|<\min \left\{\delta_{L}^{+\infty}(t), \delta_{R}^{+\infty}(t)\right\}$, then

$$
\begin{aligned}
\tilde{d}\left(x\left(t_{k}\right), B_{r}(0)\right) & <\tilde{d}\left(x\left(t_{k}\right), x\left(t_{k}^{-}\right)\right)+\tilde{d}\left(x\left(t_{k}^{-}\right), x(s)\right)+\tilde{d}\left(x(s), B_{r}(0)\right) \\
& <\varepsilon+\varepsilon+\varepsilon=3 \varepsilon,
\end{aligned}
$$

where $s \in\left(t_{k}-\delta_{L}^{+\infty}\left(t_{k}\right), t_{k}\right)_{\mathbb{T}} \subset\left(t-\delta_{L}^{+\infty}(t), t+\delta_{R}^{+\infty}(t)\right)_{\mathbb{T}}, k \in \mathbb{Z}$. This completes the proof.

Lemma 4.1 Let functions $x, y, z: \mathbb{T} \rightarrow \mathbb{R}^{n}$. Then for any $a \in \mathbb{R}^{+} \cup\{0\}$, the function $[x(\cdot), y(\cdot)]_{\mu}$ has the following properties:

(i) $\left|[x(t), y(t)]_{a \mu}\right| \leq|y(t)|$.

(ii) $[x(t), y(t)+z(t)]_{a \mu} \leq[x(t), y(t)]_{2 a \mu}+[x(t), z(t)]_{2 a \mu}$.

(iii) Let $u(t)$ be a function from an interval $J \subset \mathbb{T}$ into $\mathbb{R}^{n}$ such that $u^{\Delta}\left(t_{0}\right)$ exists for an interior point $t_{0}$ of $J$. Then $D_{+}\left|u\left(t_{0}\right)\right|^{\Delta}$ exists and

$$
D_{+}\left|u\left(t_{0}\right)\right|^{\Delta}=\left[u\left(t_{0}\right), u^{\Delta}\left(t_{0}\right)\right]_{\mu},
$$

where $D_{+}\left|u\left(t_{0}\right)\right|^{\Delta}$ denotes the right derivative of $|u(t)|$ at $t_{0}$. Further, for any $a \in \mathbb{R}^{+} \cup\{0\}$, we have $\left[u\left(t_{0}\right), u^{\Delta}\left(t_{0}\right)\right]_{\mu} \leq D_{+}\left|u\left(t_{0}\right)\right|^{\Delta}$.

Proof If $a=0$, then $a \mu=0$, from Remark 4.1, the results (i)-(iii) are obvious. If $a \in \mathbb{R}^{+}$, we can conclude the following:

(i) In fact, if $t$ is a right-dense point, by (4.2), the result is obvious. Let $t$ be a right-scattered point, then

$$
\begin{aligned}
\left|[x(t), y(t)]_{a \mu}\right| & =\left|\frac{|x(t)+a \mu(t) y(t)|-|x(t)|}{a \mu(t)}\right| \\
& \leq\left|\frac{|x(t)+a \mu(t) y(t)-x(t)|}{a \mu(t)}\right|=|y(t)| .
\end{aligned}
$$


(ii) If $t$ is a right-dense point, by (4.2), the result is obvious. Let $t$ be a right-scattered point, then

$$
\begin{aligned}
{[x(t), y(t)+z(t)]_{a \mu} } & =\frac{|x(t)+a \mu(t)(y(t)+z(t))|-|x(t)|}{a \mu(t)} \\
& =\frac{\left|\frac{1}{2} x(t)+a \mu(t) y(t)+\frac{1}{2} x(t)+a \mu(t) z(t)\right|-|x(t)|}{a \mu(t)} \\
& \leq \frac{\frac{1}{2}|x(t)+2 a \mu(t) y(t)|+\frac{1}{2}|x(t)+2 a \mu(t) z(t)|-|x(t)|}{a \mu(t)} \\
& =\frac{(|x(t)+2 a \mu(t) y(t)|-|x(t)|)+(|x(t)+2 a \mu(t) z(t)|-|x(t)|)}{2 a \mu(t)} \\
& =\frac{|x(t)+2 a \mu(t) y(t)|-|x(t)|}{2 a \mu(t)}+\frac{|x(t)+2 a \mu(t) z(t)|-|x(t)|}{2 a \mu(t)} \\
& =[x(t), y(t)]_{2 a \mu}+[x(t), z(t)]_{2 a \mu} .
\end{aligned}
$$

(iii) If $t_{0}$ is a right-dense point, by (4.2), the result is obvious. Let $t_{0}$ be a right-scattered point, then

$$
\begin{aligned}
{\left[u\left(t_{0}\right), u^{\Delta}\left(t_{0}\right)\right]_{\mu} } & =\frac{\left|u\left(t_{0}\right)+\mu\left(t_{0}\right) u^{\Delta}\left(t_{0}\right)\right|-\left|u\left(t_{0}\right)\right|}{\mu\left(t_{0}\right)} \\
& =\frac{\left|u\left(t_{0}\right)+\mu\left(t_{0}\right) \frac{u\left(\sigma\left(t_{0}\right)\right)-u\left(t_{0}\right)}{\mu\left(t_{0}\right)}\right|-\left|u\left(t_{0}\right)\right|}{\mu\left(t_{0}\right)} \\
& =\frac{\left|u\left(\sigma\left(t_{0}\right)\right)\right|-\left|u\left(t_{0}\right)\right|}{\mu\left(t_{0}\right)}=D_{+}\left|u\left(t_{0}\right)\right|^{\Delta} .
\end{aligned}
$$

Further, for $a \in \mathbb{R}^{+}$, we can obtain

$$
\begin{aligned}
{\left[u\left(t_{0}\right), u^{\Delta}\left(t_{0}\right)\right]_{a \mu} } & =\frac{\left|u\left(t_{0}\right)+a \mu\left(t_{0}\right) u^{\Delta}\left(t_{0}\right)\right|-\left|u\left(t_{0}\right)\right|}{a \mu\left(t_{0}\right)} \\
& =\frac{\left|u\left(t_{0}\right)+a \mu\left(t_{0}\right) \frac{u\left(\sigma\left(t_{0}\right)\right)-u\left(t_{0}\right)}{\mu\left(t_{0}\right)}\right|-\left|u\left(t_{0}\right)\right|}{a \mu\left(t_{0}\right)} \\
& =\frac{\left|a u\left(\sigma\left(t_{0}\right)\right)-a u\left(t_{0}\right)+u\left(t_{0}\right)\right|-\left|u\left(t_{0}\right)\right|}{a \mu\left(t_{0}\right)} \\
& \leq \frac{\left|a u\left(\sigma\left(t_{0}\right)\right)-a u\left(t_{0}\right)+u\left(t_{0}\right)-u\left(t_{0}\right)\right|}{a \mu\left(t_{0}\right)}=D_{+}\left|u\left(t_{0}\right)\right|^{\Delta} .
\end{aligned}
$$

This completes the proof.

From Definition 4.1, we obtain the following theorem, which guarantees that (4.3) has a unique global solution on $\mathbb{T}$.

Theorem 4.2 Let $A \in P C_{r d}\left(\mathbb{T} \times \mathbb{R}^{n}, \mathbb{R}^{n}\right), g \in P C_{r d}\left(\mathbb{T}, \mathbb{R}^{n}\right)$ be bounded, $A(t, \mathbf{0})=\mathbf{0}$ for all $t \in \mathbb{T}$ and $I_{k}(\mathbf{0})=\mathbf{0}$, $\Pi$ be relatively dense in $\mathbb{R}$. Suppose that there exist positive numbers $p>M>0, r>0$ such that $|g(t)| \leq M, p>\frac{M}{r}$ and

$$
[x-y, A(t, x)-A(t, y)]_{a \mu} \leq-p|x-y|
$$


for $t \in\left(t_{k-1}, t_{k}\right), k \in \mathbb{Z},-p \in \mathcal{R}^{+}, a \in \mathbb{R}^{+} \cup\{0\},|x(t)| \leq r,|y(t)| \leq r$, and

$$
\left|\tilde{I}_{k}(x)-\tilde{I}_{k}(y)\right| \leq \tilde{I}|x-y|, \quad \forall x, y \in \mathbb{R}^{n}
$$

and $\tilde{h}=\inf \left(t_{k}-t_{k-1}\right)>\frac{\ln (1+\tilde{I})}{M}, \tilde{I}$ is a Lipschitz constant. Then (4.3) has a unique solution $u(t)$ with respect to the set of solution of (4.3) in $C_{r}$, where $C_{r}=\left\{\varphi \in P C_{r d}\left(\mathbb{T}, \mathbb{R}^{n}\right):|\varphi(t)| \leq\right.$ $r$ for all $t \in \mathbb{T}\}$. Moreover, if $v(t)$ is any solution of (4.3) such that, for some $t_{0} \in \mathbb{T},\left|v\left(t_{0}\right)\right| \leq$ $\frac{M}{p}$, then $|v(t)| \leq r$ and

$$
|v(t)-u(t)| \leq\left|v\left(t_{0}\right)-u\left(t_{0}\right)\right| \prod_{t_{0}<t_{k}<t}(1+\tilde{I}) e_{-p}\left(t, t_{0}\right)
$$

for all $t \geq t_{0}$.

Proof We fix a vector $u_{0} \in \mathbb{R}^{n}$ with $\left|u_{0}\right| \leq \frac{M}{p}$. For each positive $t_{0}^{n} \in \mathbb{T}(n \in \mathbb{N})$, we consider the Cauchy problem for (4.3) with the initial value $x\left(t_{0}^{n}\right)=u_{0}$.

For each $x \in \mathbb{R}^{n}$ with $|x|=r$, for $t \in\left(t_{k-1}, t_{k}\right)_{\mathbb{T}}, k \in \mathbb{Z}$, (4.10) and (ii) in Lemma 4.1 imply

$$
[x, A(t, x)+g(t)]_{\mu} \leq[x, A(t, x)]_{2 \mu}+|g(t)| \leq-p|x|+M=-p r+M<0 .
$$

So for any $\varepsilon>0$, there exists a neighborhood of $U$ such that

$$
\begin{aligned}
|x+| \sigma(t)-s|(A(t, x)+g(t))-| x||< & |x+| \sigma(t)-s|(A(t, x)+g(t))-| x \mid \\
& -|\sigma(t)-s|[x, A(t, x)+g(t)]_{\mu} \mid \\
< & \varepsilon|\sigma(t)-s|,
\end{aligned}
$$

which implies that

$$
\tilde{d}\left(x+|\sigma(t)-s|(A(t, x)+g(t)), B_{r}(0)\right)<\varepsilon|\sigma(t)-s|,
$$

by Theorem 4.1, and we see that (4.3) has a unique global solution $u_{n}(t)$ on $\mathbb{T}$ such that $u_{n} \in B_{r}(0)$ for all $t \in \mathbb{T}$. Moreover, we can show that $\left|u_{n}(t)\right| \leq r$ for all $t \in \mathbb{T}$. In fact, (4.10) and (iii) from Lemma 4.1 imply

$$
D_{+}\left|u_{n}(t)\right|^{\Delta}=\left[u_{n}(t), A\left(t, u_{n}(t)\right)+g(t)\right]_{\mu} \leq-p\left|u_{n}(t)\right|+|g(t)| \leq-p\left|u_{n}(t)\right|+M
$$

for $t \in\left(t_{k-1}, t_{k}\right)_{\mathbb{T}}, k \in \mathbb{Z}$. By (4.11), solving the differential inequality (4.12), we obtain

$$
\begin{aligned}
\left|u_{n}(t)\right| & \leq\left|u_{n}\left(t_{0}^{n}\right)\right| e_{-p}\left(t, t_{0}^{n}\right)+M \int_{t_{0}^{n}}^{t} e_{-p}(t, \sigma(s)) \Delta s+\sum_{t_{0}^{n}<t_{k}<t} e_{-p}\left(t, t_{k}\right) \tilde{I}_{k}\left(u_{n}\left(t_{k}\right)\right) \\
& \leq \frac{M}{p} e_{-p}\left(t, t_{0}^{n}\right)+\left(-\frac{M}{p}\right)\left[e_{-p}\left(t, t_{0}^{n}\right)-e_{-p}(t, t)\right]+\tilde{I} \sum_{t_{0}^{n}<t_{k}<t} e^{-p\left(t-t_{k}\right)} \\
& \leq \frac{M}{p}+\frac{\tilde{I}}{1-e^{-p \theta}} \leq \frac{M}{p}+\frac{\tilde{I}}{1-e_{\ominus p}(\theta, 0)}:=r
\end{aligned}
$$


for all $t \in \mathbb{T}$, we always denote that $\sup _{k} e_{\ominus p}\left(t_{k+1}, t_{k}\right):=e_{\ominus p}(\theta, 0)$. According to Corollary 3.3, there exists a subsequence $\left\{u_{n_{k}}\right\}$ such that $u_{n_{k}} \rightarrow u$ on $\mathbb{T}$ as $k \rightarrow+\infty$. Therefore, there exists a solution $u(t)$ of (4.3) such that $|u(t)| \leq r$ for all $t \in \mathbb{T}$.

We next show that this solution $u(t)$ is unique with respect to the set of solutions of (4.3) in $C_{r}$. Let $v(t)$ be another solution of (4.3) in $C_{r}$ and let $w(t)=|u(t)-v(t)|$ for $t \in \mathbb{T}$. Then by (iii) from Lemma 4.1 and (4.10), we have

$$
\begin{aligned}
D_{+} w^{\Delta}(t) & =[u(t)-v(t), A(t, u(t))-A(t, v(t))]_{\mu} \\
& \leq-p|u(t)-v(t)|=-p w(t)
\end{aligned}
$$

for $t \in\left(t_{k-1}, t_{k}\right)_{\mathbb{T}}$. This implies that $w(t)$ is a nonincreasing function on $\left(t_{k-1}, t_{k}\right)_{\mathbb{T}}$. Hence, for $t_{0} \in \mathbb{T}, t \geq t_{0}$, we obtain

$$
w(t) \leq w\left(t_{0}\right)-p \int_{t_{0}}^{t} w(s) \Delta s+\tilde{I} \sum_{t_{0}<t_{k}<t} w\left(t_{k}\right),
$$

and by Gronwall-Bellman's inequality on time scales, from (4.13), we obtain

$$
w(t) \leq w\left(t_{0}\right) \prod_{t_{0}<t_{k}<t}(1+\tilde{I}) e_{-p}\left(t, t_{0}\right) .
$$

Suppose, for contradiction, that $w\left(t_{0}\right)>0$ for some $t_{0} \in \mathbb{T}$. Since $w(t)$ is bounded and is increasing as $t$ decreases, $\lim _{t \rightarrow-\infty} w(t)$ exists. For $\Pi$ is relatively dense in $\mathbb{R}$, which implies that, for each $\varepsilon>0$ and $h_{1}>0,-h_{1} \in \Pi$, there exists $t_{1} \leq 0, t_{1} \in \mathbb{T} \cap \mathbb{T}^{-h_{1}}$ such that $t_{1}+h_{1}<t_{0}$ and

$$
w\left(t_{1}\right)-w\left(t_{1}+h_{1}\right)<\varepsilon h_{1} .
$$

Set $h_{2} \leq \frac{h_{1}}{2}$ and $-h_{2} \in \Pi$ such that $t_{1} \in \mathbb{T} \cap \mathbb{T}^{-h_{2}}$. Then we have either

$$
w\left(t_{1}\right)-w\left(t_{1}+h_{2}\right)<\varepsilon h_{2}
$$

or

$$
w\left(t_{1}+h_{2}\right)-w\left(t_{1}+h_{1}\right)<\varepsilon h_{2} .
$$

Hence there exists $t_{2} \in \mathbb{T} \cap \mathbb{T}^{-h_{2}}\left(t_{2}=t_{1}\right.$ or $\left.t_{1}+h_{2}\right)$ such that $t_{2}, t_{2}+h_{2} \in\left[t_{1}, t_{1}+h_{1}\right]_{\mathbb{T}}$ and

$$
w\left(t_{2}\right)-w\left(t_{2}+h_{2}\right)<\varepsilon h_{2} .
$$

Repeating this procedure, we have sequences $\left\{t_{n}\right\},\left\{t_{n}+h_{n}\right\}$ such that $t_{n}, t_{n}+h_{n} \in\left[t_{1}, t_{1}+h_{1}\right]_{\mathbb{T}}$ and

$$
w\left(t_{n}\right)-w\left(t_{n}+h_{n}\right)<\varepsilon h_{n}, \quad \text { for } n \geq 1 \text {. }
$$


On the other hand, by (4.14), we have

$$
\begin{aligned}
w\left(t_{n}+h_{n}\right) & \leq w\left(t_{n}\right) \prod_{t_{n}<t_{k}<t_{n}+h_{n}}(1+\tilde{I}) e_{-p}\left(t_{n}+h_{n}, t_{n}\right) \\
& \leq\left(w\left(t_{n}+h_{n}\right)+\varepsilon h_{n}\right) \prod_{t_{n}<t_{k}<t_{n}+h_{n}}(1+\tilde{I}) e_{-p}\left(t_{n}+h_{n}, t_{n}\right) \\
& \leq w\left(t_{n}+h_{n}\right) \prod_{t_{n}<t_{k}<t_{n}+h_{n}}(1+\tilde{I}) e_{-p}\left(t_{n}+h_{n}, t_{n}\right)+\varepsilon h_{n}
\end{aligned}
$$

for $n \geq 1$. Therefore, it follows that

$$
\begin{aligned}
w\left(t_{0}\right) & \leq w\left(t_{n}+h_{n}\right) \leq\left\{\frac{h_{n}}{1-\prod_{t_{n}<t_{k}<t_{n}+h_{n}}(1+\tilde{I}) e_{-p}\left(t_{n}+h_{n}, t_{n}\right)}\right\} \varepsilon \\
& \leq\left\{\frac{h_{n}}{1-\prod_{t_{n}<t_{k}<t_{n}+h_{n}}(1+\tilde{I}) e^{-p h_{n}}}\right\} \varepsilon \\
& \leq\left\{\frac{h_{n}}{1-\prod_{t_{n}<t_{k}<t_{n}+h_{n}}(1+\tilde{I}) e^{-p\left(t_{k+1}-t_{k}\right)}}\right\} \varepsilon \quad(n \geq 1) .
\end{aligned}
$$

Next, we will show that

$$
\prod_{t_{n}<t_{k}<t_{n}+h_{n}}(1+\tilde{I}) e^{-p\left(t_{k+1}-t_{k}\right)}<1 .
$$

Case $I$. If $\left[t_{n}, t_{n}+h_{n}\right]_{\mathbb{T}}$ does not contain any $t_{k}, k \in \mathbb{Z}$, then $t_{k+1}-t_{k}>h_{n},(4.16)$ becomes

$$
\prod_{t_{n}<t_{k}<t_{n}+h_{n}} e^{-p\left(t_{k+1}-t_{k}\right)}=e^{-p\left(t_{k+1}-t_{k}\right)}<e^{-p h_{n}}<1 .
$$

Case II. If $\left[t_{n}, t_{n}+h_{n}\right]_{\mathbb{T}}$ contains $n_{0}$ impulsive points $t_{k}, k \in \mathbb{S}=\left\{0,1, \ldots, n_{0}\right\}$, then

$$
\begin{aligned}
\prod_{t_{n}<t_{k}<t_{n}+h_{n}} \frac{(1+\tilde{I})}{e^{p\left(t_{k+1}-t_{k}\right)}} & <\prod_{t_{n}<t_{k}<t_{n}+h_{n}} \frac{(1+\tilde{I})}{e^{M\left(t_{k+1}-t_{k}\right)}}<\frac{(1+\tilde{I})^{n_{0}}}{\prod_{t_{n}<t_{k}<t_{n}+h_{n}} e^{\left(t_{k}-t_{k-1}\right) M}} \\
& <\left(\frac{1+\tilde{I}}{e^{\tilde{h} M}}\right)^{n_{0}}<\left(\frac{1+\tilde{I}}{e^{\tilde{h} M}}\right)^{n_{0}}<1 \quad \text { since } \tilde{h}>\frac{\ln (1+\tilde{I})}{M} .
\end{aligned}
$$

Hence, from (4.15), let $n \rightarrow \infty$, we have $w\left(t_{0}\right)=0$. This leads to a contradiction. The last assertion of this theorem follows easily from the preceding argument. The proof is complete.

In the following, let $\mathbb{T}$ be an almost periodic time scale. For convenience, we give some notation, $B C$ denotes the set of $\mathbb{R}^{n}$-valued functions that is continuous and bounded on $\left(-\infty, s_{0}\right]_{\Pi_{\varepsilon}}, s_{0} \in \Pi_{\varepsilon}$; for each $\phi \in B C$ we define $\|\phi\|_{\varepsilon}=\sup _{s \in\left(-\infty, s_{0}\right]_{\Pi_{\varepsilon}}}|\phi(s)|$. Then $\left(B C,\|\cdot\|_{\varepsilon}\right)$ is a real Banach space for any fixed $\varepsilon>0$.

Remark 4.2 From the definition of $\|\phi\|_{\varepsilon}$, if we introduce a set

$$
\tilde{\Omega}=\left\{\phi:\|\phi\|_{\varepsilon} \leq r, \forall \varepsilon>0, r \text { is a constant }\right\}
$$

then for $\forall \phi \in \tilde{\Omega}$, one can immediately obtain that $\|\phi\|_{\varepsilon_{1}} \leq\|\phi\|_{\varepsilon_{2}} \leq r$ if $\varepsilon_{1}<\varepsilon_{2}$. 
Let $x(t)$ be an $\mathbb{R}^{n}$-valued function that is continuous and bounded on $\mathbb{T}$, we define for each $t \in \mathbb{T} \cap\left(\cup \mathbb{T}^{\Pi_{\varepsilon}}\right), x_{t}(s)=x(t+s)$ for $-s \in \Pi_{\varepsilon}$. Clearly, $x_{t} \in B C$ for each $t \in \mathbb{T} \cap\left(\cup \mathbb{T}^{\Pi_{\varepsilon}}\right)$.

We now consider the $\varepsilon$-equivalent impulsive functional dynamic equation

$$
\begin{cases}x^{\Delta}(t)=F\left(t, x(t), x_{t}\right), & t \neq t_{k}, t \in \mathbb{T} \cap\left(\cup \mathbb{T}^{\Pi_{\varepsilon}}\right), \\ \Delta x\left(t_{k}\right)=I_{k}\left(x\left(t_{k}\right)\right), & t=t_{k}, k \in \mathbb{Z}\end{cases}
$$

$F(t, x, \phi)$ is the $\mathbb{R}^{n}$-valued function defined on $\mathbb{T} \times \mathbb{R}^{n} \times B C$ which satisfies the following conditions:

$\left(\mathrm{H}_{1}\right)$ for each $r>0$, there exists $M(r)>0$ and $N(r)>0$ such that

$$
|F(t, 0, \phi)| \leq M(r) \quad \text { and } \quad|F(t, x, \phi)| \leq N(r)
$$

for all $t \in \mathbb{T},|x| \leq r$ and $\|\phi\|_{\varepsilon} \leq r(\phi \in B C)$. Moreover, for any $x, y \in \mathbb{R}^{n}$,

$$
\left|I_{k}(x)-I_{k}(y)\right| \leq \tilde{N}(r)|x-y|, \quad I_{k}(\mathbf{0})=\mathbf{0},
$$

and $\tilde{h}=\inf \left(t_{k}-t_{k-1}\right)>\frac{\ln (1+\tilde{N}(r))}{M(r)}$

$\left(\mathrm{H}_{2}\right)$ if for $x(t)$ that is piecewise continuous and bounded on $\mathbb{T}, F\left(t, y, x_{t}\right)$ is piecewise continuous in $(t, y)$ on $\mathbb{T} \times \mathbb{R}^{n}$;

$\left(\mathrm{H}_{3}\right)$ if there exist positive numbers $p, r, L$ such that $p>\max \left\{\frac{M(r)}{r}, L\right\}$, where $M(r)$ is as in $\left(\mathrm{H}_{1}\right)$, such that

(i) $[x-y, F(t, x, \phi)-F(t, y, \phi)]_{a \mu} \leq-p|x-y|$ for $t \in \mathbb{T},|x| \leq r,|y| \leq r,\|\phi\|_{\varepsilon} \leq r(\phi \in B C), a \in \mathbb{R}^{+} ;$

(ii) $\left[\phi(0)-\phi^{*}(0), F(t, \phi(0), \phi)-F\left(t, \phi^{*}(0), \phi^{*}\right)\right]_{a \mu} \leq-p\left|\phi(0)-\phi^{*}(0)\right|+L\left\|\phi-\phi^{*}\right\|_{\varepsilon}$ for $t \in \mathbb{T},\|\phi\|_{\varepsilon} \leq r,\left\|\phi^{*}\right\|_{\varepsilon} \leq r\left(\phi, \phi^{*} \in B C\right), a \in \mathbb{R}^{+} \cup\{0\}$;

(iii) $\frac{\tilde{N}(r)}{1-e_{-}(\theta, 0)}+\frac{L}{p}<1$, where $\sup _{k} e_{-p}\left(t_{k+1}, t_{k}\right):=e_{-p}(\theta, 0)$;

$\left(\mathrm{H}_{4}\right)$ if for $x^{k}(t), y^{k}(t), x(t)$ and $y(t)$ are piecewise continuous such that $\left|x^{k}(t)\right| \leq r,\left|y^{k}(t)\right| \leq r$ for all $t \in \mathbb{T}, k \geq 1$ and $x^{k}(t) \rightarrow x(t), y^{k}(t) \rightarrow y(t)$ as $k \rightarrow \infty$ for $t \in \mathbb{T}$, we have

$$
F\left(t, x^{k}(t), y_{t}^{k}\right) \rightarrow F\left(t, x(t), y_{t}\right) \quad \text { as } k \rightarrow \infty \text { for } t \in \mathbb{T} \cap\left(\cup \mathbb{T}^{\Pi_{\varepsilon}}\right)
$$

Remark 4.3 In $\left(\mathrm{H}_{2}\right)$, piecewise continuity of $F\left(t, y, x_{t}\right)$ in $(t, y)$ on $\mathbb{T} \times \mathbb{R}^{n}$ implies piecewise continuity of $F\left(t, x(t), x_{t}\right)$ in $t$ on $\mathbb{T}$. Moreover, if $F$ is Lipschitz continuous in $x$ uniformly for $t \in \mathbb{T}, \phi \in B C\left(\|\phi\|_{\varepsilon} \leq r\right)$ with Lipschitz constant $K$, then we can take $N(r)=K r+M(r)$.

Theorem 4.3 Suppose that $\left(\mathrm{H}_{1}\right)-\left(\mathrm{H}_{4}\right)$ are satisfied and

$$
|F(t, x, \phi)-F(t, y, \phi)| \leq K|x-y|, \quad \forall x, y \in \mathbb{R}^{n}
$$

where $K$ is a Lipschitz constant. Then there exists a solution $u(t)$ of (4.17) such that $|u(t)| \leq r$ for all $t \in \mathbb{T} \cap\left(\cup \mathbb{T}^{\Pi_{\varepsilon}}\right)$, and this solution is unique in $\left.C_{r}^{*}\right|_{\mathbb{T} \cap\left(\cup \mathbb{T}_{\varepsilon}\right)}$. Here $r$ is as in $\left(\mathrm{H}_{3}\right), C_{r}^{*}=\left\{\varphi \in P C_{r d}\left(\mathbb{T}, \mathbb{R}^{n}\right): \varphi(t)\right.$ is piecewise continuous and $|\varphi(t)| \leq r$ for all $\left.t \in \mathbb{T}\right\}$, and $\left.C_{r}^{*}\right|_{\mathbb{T} \cap\left(\cup \mathbb{T} \Pi_{\varepsilon}\right)}=\left\{\left.\varphi\right|_{\mathbb{T} \cap\left(\cup \mathbb{T} \Pi_{\varepsilon}\right)}: \forall \varphi \in C_{r}^{*}\right\}$. 
Proof Let $N(r)$ be as in $\left(\mathrm{H}_{1}\right)$ and set $S_{r}=\left\{f \in P C_{r d}\left(\mathbb{T}, \mathbb{R}^{n}\right):|f(t)| \leq r\right.$ and $\left|f^{\Delta}(t)\right| \leq K r+$ $M(r)\}$. Obviously, $S_{r}$ is a compact convex subset of $P C_{r d}\left(\mathbb{T}, \mathbb{R}^{n}\right)$. We set

$$
A(t, x, \phi)=F(t, x, \phi)-F(t, 0, \phi) \quad \text { and } \quad B(t, \phi)=F(t, 0, \phi) .
$$

Define a mapping $T: S_{r} \rightarrow S_{r}$ as follows: $x(t)=T f(t)$ is the unique solution in $C_{r}$ of

$$
\begin{cases}x^{\Delta}(t)=A\left(t, x(t), f_{t}\right)+B\left(t, f_{t}\right), & t \in \mathbb{T}, t \neq t_{k} \\ \Delta x\left(t_{k}\right)=I_{k}\left(x\left(t_{k}\right)\right), & t=t_{k}, k \in \mathbb{Z}\end{cases}
$$

where $C_{r}$ is as in Theorem 4.2 and $f \in S_{r}$. Such a solution $x(t)$ exists by Theorem 4.2 and satisfies $|x(t)| \leq r$ for all $t \in \mathbb{T}$. Since

$$
\begin{aligned}
\left|x^{\Delta}(t)\right| & =|F(t, x, \phi)-F(t, 0, \phi)+F(t, 0, \phi)| \\
& \leq|F(t, x, \phi)-F(t, 0, \phi)|+|F(t, 0, \phi)| \leq K r+M(r)
\end{aligned}
$$

it follows that $x \in S_{r}$.

We show next that $T$ is continuous on $S_{r}$. Let $f^{k}$ and $f$ be in $S_{r}$ such that $f^{k} \rightarrow f$ as $k \rightarrow \infty$. Put $x^{k}=T f^{k}$ and $x=T f$. We show that each sequence of $\left\{x^{k}\right\}$ contains a subsequence which converges to $x$. This will show that $T$ is continuous at $f \in S_{r}$. Let us denote this arbitrary subsequence again by $\left\{x^{k}\right\}$ for simplicity. Since $x^{k} \in S_{r},\left\{x^{k}\right\}$ is $\Delta$-differentiable almost everywhere on $\mathbb{T}$ and uniformly bounded on $\mathbb{T}$. According to Corollary 3.3 , there exists a subsequence $\left\{x^{k_{j}}\right\}$ of $\left\{x^{k}\right\}$ and a $w \in S_{r}$ such that $x^{k_{j}}(t) \rightarrow w(t)$ as $j \rightarrow \infty$ uniformly on $\mathbb{T}$. Let $t_{0} \in \mathbb{T} \cap\left(\cup \mathbb{T}^{\Pi_{\varepsilon}}\right)$ and let

$$
w^{*}(t)=w\left(t_{0}\right)+\int_{t_{0}}^{t} F\left(s, w(s), f_{s}\right) \Delta s+\sum_{t_{0}<t_{k}<t} I_{k}\left(w\left(t_{k}\right)\right) \quad \text { for } t \in \mathbb{T} \cap\left(\cup \mathbb{T}^{\Pi_{\varepsilon}}\right) .
$$

Then we have

$$
\begin{aligned}
\left|x^{k_{j}}(t)-w^{*}(t)\right|= & \mid x^{k_{j}}\left(t_{0}\right)+\int_{t_{0}}^{t} F\left(s, x^{k_{j}}(s), f_{s}^{k_{j}}\right) \Delta s-w\left(t_{0}\right)-\int_{t_{0}}^{t} F\left(s, w(s), f_{s}\right) \Delta s \\
& +\sum_{t_{0}<t_{k}<t} I_{k}\left(x^{k_{j}}\left(t_{k}\right)\right)-\sum_{t_{0}<t_{k}<t} I_{k}\left(w\left(t_{k}\right)\right) \mid \\
\leq & \left|x^{k_{j}}\left(t_{0}\right)-w\left(t_{0}\right)\right|+\int_{t_{0}}^{t}\left|F\left(s, x^{k_{j}}(s), f_{s}^{k_{j}}\right)-F\left(s, w(s), f_{s}\right)\right| \Delta s \\
& +\sum_{t_{0}<t_{k}<t} \tilde{N}(r)\left|x^{k_{j}}\left(t_{k}\right)-w\left(t_{k}\right)\right|
\end{aligned}
$$

for $t \in \mathbb{T} \cap\left(\cup \mathbb{T}^{\Pi_{\varepsilon}}\right)$. By $\left(\mathrm{H}_{1}\right),\left(\mathrm{H}_{2}\right),\left(\mathrm{H}_{4}\right)$, and the Lebesgue dominated convergence theorem we can see that $x^{k_{j}}(t) \rightarrow w^{*}(t)$ as $j \rightarrow \infty$ for $t \in \mathbb{T}$, i.e., $w^{*}(t)=w(t)$ for $t \in \mathbb{T} \cap\left(\cup \mathbb{T}^{\Pi_{\varepsilon}}\right)$. Hence, $w$ is a solution for (4.18) on $\left.C_{r}^{*}\right|_{\mathbb{T} \cap\left(\cup \mathbb{T} \Pi_{\varepsilon}\right)}$. By the uniqueness of solutions of (4.18), it follows that $w(t)=x(t)$ for $t \in \mathbb{T} \cap\left(\cup \mathbb{T}^{\Pi_{\varepsilon}}\right)$. Thus we conclude that $T$ is continuous at each 
$f \in S_{r}$. From Lemma 2.3, there exists a $u \in S_{r}$ such that $T u=u$, i.e.,

$$
\begin{cases}u^{\Delta}(t)=A\left(t, u(t), u_{t}\right)+B\left(t, u_{t}\right)=F\left(t, u(t), u_{t}\right), & t \in \mathbb{T} \cap\left(\cup \mathbb{T}^{\Pi_{\varepsilon}}\right), t \neq t_{k}, \\ \Delta u\left(t_{k}\right)=I_{k}\left(u\left(t_{k}\right)\right), & t=t_{k}, k \in \mathbb{Z} .\end{cases}
$$

To prove the uniqueness of the solution of (4.17), let $\left.v \in C_{r}^{*}\right|_{\mathbb{T} \cap\left(\cup \mathbb{T} \Pi_{\varepsilon}\right)}$ be another solution of (4.17). By (ii), (iii) in $\left(\mathrm{H}_{3}\right)$, and (iii) in Lemma 4.1, we have

$$
\begin{aligned}
D_{+}|u(t)-v(t)|^{\Delta} & =\left[u(t)-v(t), F\left(t, u(t), u_{t}\right)-F\left(t, v(t), v_{t}\right)\right]_{\mu} \\
& \leq-p|u(t)-v(t)|+L\left\|u_{t}-v_{t}\right\|_{\varepsilon}
\end{aligned}
$$

for all $t \in\left(t_{k-1}, t_{k}\right)_{\mathbb{T}} \cap\left(\cup \mathbb{T}^{\Pi_{\varepsilon}}\right), k \in \mathbb{Z}$. Let $\psi(t)=|u(t)-v(t)|$, then we obtain

$$
\left\{\begin{array}{l}
D_{+} \psi(t)^{\Delta} \leq-p \psi(t)+L\left\|\psi_{t}\right\|_{\varepsilon} \\
\psi\left(t_{k}^{+}\right) \leq \psi\left(t_{k}^{-}\right)+\tilde{N}(r) \psi\left(t_{k}\right)
\end{array}\right.
$$

Then for $t \in\left[t_{k}, t_{k+1}\right)_{\mathbb{T}} \cap\left(\cup \mathbb{T}^{\Pi_{\varepsilon}}\right), k \in \mathbb{Z}$, we get

$$
\psi(t) \leq \psi\left(t_{k}^{+}\right) e_{-p}\left(t, t_{k}\right)+\int_{t_{k}}^{t} e_{-p}(t, \sigma(s)) L\left\|\psi_{s}\right\|_{\varepsilon} \Delta s
$$

thus we obtain

$$
\begin{aligned}
& \psi(t) \leq \psi\left(t_{k}^{-}\right) e_{-p}\left(t, t_{k}\right)+\int_{t_{k}}^{t} e_{-p}(t, \sigma(s)) L\left\|\psi_{s}\right\|_{\varepsilon} \Delta s+\tilde{N}(r) \psi\left(t_{k}\right) e_{-p}\left(t, t_{k}\right), \\
& \psi\left(t_{k+1}^{-}\right) \leq \psi\left(t_{k}^{+}\right) e_{-p}\left(t_{k+1}, t_{k}\right)+\int_{t_{k}}^{t_{k+1}} e_{-p}\left(t_{k+1}, \sigma(s)\right) L\left\|\psi_{s}\right\|_{\varepsilon} \Delta s
\end{aligned}
$$

Note that $u(t)-v(t)=u_{t}(0)-v_{t}(0)$, from (4.20) and (4.21), for $t \in\left[t_{0}+s, t+s\right)_{\mathbb{T}} \cap\left(\cup \mathbb{T}^{\Pi_{\varepsilon}}\right)$, solving the differential inequality (4.19), we have

$$
\begin{aligned}
\psi(t+s) \leq & \psi\left(t_{0}+s\right) \prod_{t_{0}+s<t_{k}<t+s} e_{-p}\left(t+s, t_{0}+s\right) \\
& +\sum_{t_{0}+s<t_{k}<t+s}\left(\prod_{t_{k}<t_{j}<t+s} e_{-p}\left(t+s, t_{k}\right)\right) \tilde{N}(r) \psi\left(t_{k}\right) \\
& +\int_{t_{0}+s}^{t+s} \prod_{\theta<t_{k}<t+s} e_{-p}(t+s, \sigma(\theta)) L\left\|\psi_{\theta}\right\|_{\varepsilon} \Delta \theta
\end{aligned}
$$

for all $t \in\left[t_{0},+\infty\right)_{\mathbb{T}} \cap\left(\cup \mathbb{T}^{\Pi_{\varepsilon}}\right),-s \in \Pi_{\varepsilon}$. Note $\left\|\psi_{\theta}\right\|_{\varepsilon}=\left\|u_{\theta}-v_{\theta}\right\|_{\varepsilon}$ is nondecreasing function of $\theta$. It therefore follows that

$$
\begin{aligned}
\left\|u_{t}-v_{t}\right\|_{\varepsilon} \leq & \prod_{t_{0}+s<t_{k}<t+s} e_{-p}\left(t+s, t_{0}+s\right)\left\|u_{t_{0}}-v_{t_{0}}\right\|_{\varepsilon} \\
& +\left(\frac{\tilde{N}(r)}{1-e_{-p}(\theta, 0)}+\frac{L}{p}\left(1-e_{-p}\left(t+s, t_{0}+s\right)\right)\right)\left\|u_{t}-v_{t}\right\|_{\varepsilon}
\end{aligned}
$$




$$
\begin{gathered}
\leq \prod_{t_{0}+s<t_{k}<t+s} e_{-p}\left(t+s, t_{0}+s\right)\left\|u_{t_{0}}-v_{t_{0}}\right\|_{\varepsilon} \\
\quad+\left(\frac{\tilde{N}(r)}{1-e_{-p}(\theta, 0)}+\frac{L}{p}\right)\left\|u_{t}-v_{t}\right\|_{\varepsilon}
\end{gathered}
$$

for all $t \in\left[t_{0},+\infty\right)_{\mathbb{T}} \cap\left(\cup \mathbb{T}^{\Pi_{\varepsilon}}\right)$, and this implies

$$
\left\|u_{t}-v_{t}\right\|_{\varepsilon} \leq \frac{1}{1-\tilde{M}} \prod_{t_{0}+s<t_{k}<t+s} e_{-p}\left(t+s, t_{0}+s\right)\left\|u_{t_{0}}-v_{t_{0}}\right\|_{\varepsilon}
$$

for all $t \in\left[t_{0},+\infty\right)_{\mathbb{T}} \cap\left(\cup \mathbb{T}^{\Pi_{\varepsilon}}\right)$, where

$$
\tilde{M}=\frac{\tilde{N}(r)}{1-e_{-p}(\theta, 0)}+\frac{L}{p}
$$

Consequently, for each $t_{0} \in \mathbb{T} \cap\left(\cup \mathbb{T}^{\Pi_{\varepsilon}}\right)$, we have

$$
\left\|u_{t_{0}}-v_{t_{0}}\right\|_{\varepsilon} \leq\left\|u_{t}-v_{t}\right\|_{\varepsilon} \leq \frac{1}{1-\tilde{M}} \prod_{t_{0}+s<t_{k}<t+s} e_{-p}\left(t+s, t_{0}+s\right)\left\|u_{t_{0}}-v_{t_{0}}\right\|_{\varepsilon}
$$

for all $t \in\left[t_{0},+\infty\right)_{\mathbb{T}} \cap\left(\cup \mathbb{T}^{\Pi_{\varepsilon}}\right)$. Letting $t \rightarrow+\infty$ we have $\left\|u_{t_{0}}-v_{t_{0}}\right\|_{\varepsilon}=0$ and this implies in particular $u\left(t_{0}\right)=v\left(t_{0}\right)$. The proof is complete.

We next consider the existence of almost periodic solutions of (4.17) when $F$ satisfies the following condition:

$\left(\mathrm{H}_{5}\right) F(t, x, \phi)$ is almost periodic in $t$ uniformly for $(x, \phi)$ in bounded closed subsets of $\mathbb{R}^{n} \times$ $B C$.

Theorem 4.4 Suppose $\left(\mathrm{H}_{1}\right)-\left(\mathrm{H}_{5}\right)$ are satisfied and the following are fulfilled:

(i) $|F(t, x, \phi)-F(t, y, \phi)| \leq K|x-y|, \forall x, y \in \mathbb{R}^{n}$, where $K$ is a Lipschitz constant;

(ii) the impulsive operator sequence $\left\{I_{k}\right\}$ is an almost periodic sequence;

(iii) $\frac{3(p-L)\left(1-e_{-}(\theta, 0)\right)}{p\left(3-2 e_{-}(\theta, 0)\right)}<1$, where $\sup _{k} e_{-p}\left(t_{k+1}, t_{k}\right):=e_{-p}(\theta, 0)$.

Then (4.17) has a unique almost periodic solution $u(t)$ in $\left.C_{r}^{*}\right|_{\mathbb{T} \cap\left(\cup \mathbb{T} \Pi_{\varepsilon}\right)}$ for $0<r<1$. Moreover, if $p-L \geq \frac{p L r+2 M(r)}{r}$ and $v(t)$ is any solution of (4.17) with $\left\|v_{t_{0}}\right\|_{\varepsilon} \leq \frac{M(r)}{p}$ for some $t_{0} \in \mathbb{T} \cap$ $\left(\cup \mathbb{T}^{\Pi_{\varepsilon}}\right)$, then $\left\|v_{t}\right\|_{\varepsilon} \leq r$ and

$$
\left\|u_{t}-v_{t}\right\|_{\varepsilon} \leq \frac{p}{p-L} e_{-p}\left(t, t_{0}\right) \prod_{t_{0}+s<t_{k}<t+s} e_{-p}\left(t+s, t_{0}+s\right)\left\|u_{t_{0}}-v_{t_{0}}\right\|_{\varepsilon}
$$

for all $t \in\left[t_{0},+\infty\right)_{\mathbb{T}} \cap\left(\cup \mathbb{T}^{\Pi_{\varepsilon}}\right),-s \in \Pi_{\varepsilon}$.

Proof Let $u(t)$ be the unique solution of (4.17) in $\left.C_{r}^{*}\right|_{\mathbb{T} \cap\left(\cup \mathbb{T} \Pi_{\varepsilon}\right)}$ obtained in Theorem 4.3. For each $\varepsilon_{1}>0$, there exists a positive number $l\left(\varepsilon_{1}\right)$ such that any interval of length $l(\varepsilon)$ contains a $\tau=\tau\left(\varepsilon_{1}\right) \in E\left\{\varepsilon_{2}, F\right\}$ for which

$$
\left|F\left(t+\tau, u(t+\tau), u_{t+\tau}\right)-F\left(t, u(t+\tau), u_{t+\tau}\right)\right|<\varepsilon_{2}
$$


for all $t \in\left(t_{k-1}, t_{k}\right)_{\mathbb{T}} \cap \mathbb{T}^{-\tau}, \tau \in \Pi_{\varepsilon_{1}}, k \in \mathbb{Z}$. By (iii) in Lemma 4.1 and (i), (ii) in $\left(\mathrm{H}_{3}\right)$, we have

$$
\begin{aligned}
D_{+}|u(t+\tau)-u(t)|^{\Delta}= & {\left[u(t+\tau)-u(t), F\left(t+\tau, u(t+\tau), u_{t+\tau}\right)-F\left(t, u(t), u_{t}\right)\right]_{\mu} } \\
\leq & -p|u(t+\tau)-u(t)|+L\left\|u_{t+\tau}-u_{t}\right\| \\
& +\left|F\left(t+\tau, u(t+\tau), u_{t+\tau}\right)-F\left(t, u(t+\tau), u_{t+\tau}\right)\right| \\
\leq & -p|u(t+\tau)-u(t)|+L\left\|u_{t+\tau}-u_{t}\right\|_{\varepsilon_{1}}+\varepsilon_{2}
\end{aligned}
$$

for all $t \in\left(t_{k-1}, t_{k}\right)_{\mathbb{T}} \cap \mathbb{T}^{-\tau},-\tau \in \Pi_{\varepsilon_{1}}, k \in \mathbb{Z}$.

For all $t \in \mathbb{T} \cap \mathbb{T}^{-s},-s \in(-\infty, 0]_{\Pi_{\varepsilon_{1}}}$. From Lemma 2.1, we can find $\beta>0$ and $\beta \in \Pi_{\varepsilon_{1}}$ such that $-s+\beta \in \Pi_{2 \varepsilon_{1}}$. Thus we obtain

$$
d\left(\mathbb{T}^{-\tau-s+\beta}, \mathbb{T}\right)<d\left(\mathbb{T}^{-\tau-s+\beta}, \mathbb{T}^{-s+\beta}\right)+d\left(\mathbb{T}^{-s+\beta}, \mathbb{T}\right)<3 \varepsilon_{1}
$$

Let $\tilde{\varphi}(t)=|u(t+\tau)-u(t)|,(4.23)$ can be written in the form

$$
D_{+} \tilde{\varphi}^{\Delta}(t) \leq-p \tilde{\varphi}(t)+L\left\|\tilde{\varphi}_{t}\right\|_{3 \varepsilon_{1}}+\varepsilon_{2} .
$$

Moreover, since $\left\{I_{k}\right\}$ is an almost periodic sequence, there exists a $q>0$ such that $\mid I_{k+q}-$ $I_{k} \mid<\varepsilon_{2}$. Hence, we obtain

$$
\tilde{\varphi}\left(t_{k}^{+}\right)-\tilde{\varphi}\left(t_{k}^{-}\right) \leq\left|I_{k+q}\left(u\left(t_{k+q}\right)\right)-I_{k}\left(u\left(t_{k}\right)\right)\right|<\varepsilon_{2} .
$$

Then for $t \in\left[t_{k}, t_{k+1}\right)_{\mathbb{T}} \cap\left(\cup \mathbb{T}^{\Pi_{3 \varepsilon_{1}}}\right), k \in \mathbb{Z}$, we get

$$
\tilde{\varphi}(t) \leq \tilde{\varphi}\left(t_{k}^{+}\right) e_{-p}\left(t, t_{k}\right)+\int_{t_{k}}^{t} e_{-p}(t, \sigma(s))\left(L\left\|\tilde{\varphi}_{s}\right\|_{3 \varepsilon_{1}}+\varepsilon_{2}\right) \Delta s,
$$

thus we obtain

$$
\begin{aligned}
& \tilde{\varphi}(t) \leq \tilde{\varphi}\left(t_{k}^{-}\right) e_{-p}\left(t, t_{k}\right)+\int_{t_{k}}^{t} e_{-p}(t, \sigma(s))\left(L\left\|\tilde{\varphi}_{s}\right\|_{\varepsilon_{1}}+\varepsilon_{2}\right) \Delta s+\varepsilon_{2} e_{-p}\left(t, t_{k}\right), \\
& \tilde{\varphi}\left(t_{k+1}^{-}\right) \leq \tilde{\varphi}\left(t_{k}^{+}\right) e_{-p}\left(t_{k+1}, t_{k}\right)+\int_{t_{k}}^{t_{k+1}} e_{-p}\left(t_{k+1}, \sigma(s)\right)\left(L\left\|\tilde{\varphi}_{s}\right\|_{3 \varepsilon_{1}}+\varepsilon_{2}\right) \Delta s
\end{aligned}
$$

Under (4.24), for all $t \in \mathbb{T} \cap\left(\cup \mathbb{T}^{\Pi_{3 \varepsilon_{1}}}\right)$, using (4.25) and (4.26), we can solve the differential inequality (4.23) and obtain

$$
\begin{aligned}
\tilde{\varphi}(t+s) \leq \tilde{\varphi}(t+s-\beta) \prod_{t+s-\beta<t_{k}<t+s} e_{-p}(t+s, t+s-\beta) \\
+\sum_{t+s-\beta<t_{k}<t+s}\left(\prod_{t_{k}<t_{j}<t+s} e_{-p}\left(t+s, t_{k}\right)\right) \varepsilon_{2} \\
+\int_{t+s-\beta}^{t+s} \prod_{\theta<t_{k}<t+s} e_{-p}(t+s, \sigma(\theta))\left(L\left\|\tilde{\varphi}_{\theta}\right\|_{3 \varepsilon_{1}}+\varepsilon_{2}\right) \Delta \theta
\end{aligned}
$$


for all $t \in \mathbb{T} \cap\left(\cup \mathbb{T}^{\Pi_{3 \varepsilon_{1}}}\right)$. Note $\left\|\tilde{\varphi}_{\theta}\right\|_{3 \varepsilon_{1}}=\left\|u_{\theta+\tau}-u_{\theta}\right\|_{3 \varepsilon_{1}}$ is nondecreasing in $\theta$ and $u \in$ $\left.C_{r}^{*}\right|_{\mathbb{T} \cap\left(\cup \mathbb{T}^{\left.\Pi_{3 \varepsilon_{1}}\right)}\right.}$. It follows from this that

$$
\begin{aligned}
\left\|u_{t+\tau}-u_{t}\right\|_{3 \varepsilon_{1}} \leq & \prod_{t+s-\beta<t_{k}<t+s} e_{-p}(t+s, t+s-\beta)\left\|u_{t+\tau-\beta}-u_{t-\beta}\right\|_{3 \varepsilon_{1}} \\
& +\frac{L}{p}\left(1-e_{-p}(t+s, t+s-\beta)\right)\left\|u_{t+\tau}-u_{t}\right\|_{3 \varepsilon_{1}}+\frac{\varepsilon_{2}}{1-e_{-p}(\theta, 0)} \\
& +\varepsilon_{2}\left(1-e_{-p}(t+s, t+s-\beta)\right) \\
\leq & \left(\prod_{t+s-\beta<t_{k}<t+s} e_{-p}(t+s, t+s-\beta)+\frac{L}{p}\right)\left\|u_{t+\tau}-u_{t}\right\|_{3 \varepsilon_{1}} \\
& +\frac{\varepsilon_{2}}{1-e_{-p}(\theta, 0)}+\varepsilon_{2},
\end{aligned}
$$

so we can obtain

$$
\begin{aligned}
\left\|u_{t+\tau}-u_{t}\right\|_{3 \varepsilon_{1}} \leq & \frac{p}{p-L} \prod_{t+s-\beta<t_{k}<t+s} 2 r e_{-p}(t+s, t+s-\beta) \\
& +\frac{\left(2-e_{-p}(\theta, 0)\right) \varepsilon_{2} p}{\left(1-e_{-p}(\theta, 0)\right)(p-L)} .
\end{aligned}
$$

Hence, for $0<r<1$, we can choose a sufficiently large $\beta_{0}>0$ such that

$$
\prod_{t+s-\beta<t_{k}<t+s} 2 r e_{-p}(t+s, t+s-\beta)<\varepsilon_{2} .
$$

Then

$$
\left\|u_{t+\tau}-u_{t}\right\|_{3 \varepsilon_{1}} \leq\left[\frac{p}{p-L}\left(1+\frac{2-e_{-p}(\theta, 0)}{1-e_{-p}(\theta, 0)}\right)\right] \varepsilon_{2} \quad \text { for all } t \in \mathbb{T} \cap\left(\cup \mathbb{T}^{\Pi_{3 \varepsilon_{1}}}\right) .
$$

Since $\varepsilon_{1}$ is arbitrary and $\varepsilon_{2}>\varepsilon_{1}>0$, from the condition (iii) from Theorem 4.4, we obtain

$$
\left[\frac{p}{p-L}\left(1+\frac{2-e_{-p}(\theta, 0)}{1-e_{-p}(\theta, 0)}\right)\right] \varepsilon_{2}>3 \varepsilon_{1}>0
$$

by Definition 2.10, $u(t)$ is an almost periodic function on the almost periodic time scale $\mathbb{T}$.

Next, we assume that $p-L \geq \frac{p L r+2 M(r)}{r}$. Let $v(t)$ be any solution of (4.17) such that $\left\|v_{t_{0}}\right\|_{\varepsilon_{1}} \leq \frac{M(r)}{p}$ for some $t_{0} \in \mathbb{T} \cap\left(\cup \mathbb{T}^{\Pi_{\varepsilon_{1}}}\right)$. Then, by (ii), (iii) in Lemma 4.1, and (ii) in $\left(\mathrm{H}_{3}\right)$, for $t \in\left(t_{k}, t_{k+1}\right)_{\mathbb{T}} \cap\left(\cup \mathbb{T}^{\Pi_{\varepsilon_{1}}}\right)$, we have

$$
\begin{aligned}
D_{+}|v(t)|^{\Delta} & =\left[v(t), F\left(t, v(t), v_{t}\right)\right]_{\mu} \\
& \leq\left[v(t), F\left(t, v(t), v_{t}\right)-F(t, 0,0)\right]_{2 \mu}+|F(t, 0,0)| \\
& \leq-p|v(t)|+L\left\|v_{t}\right\|+M(r) .
\end{aligned}
$$

Moreover, note $I_{k}$ satisfies the Lipschitz condition for each $k$. Hence, we can obtain

$$
v\left(t_{k}^{+}\right)-v\left(t_{k}^{-}\right) \leq\left|I_{k}\left(v\left(t_{k}\right)\right)-I_{k}(\mathbf{0})\right|<L r .
$$


Then for $t \in\left[t_{k}, t_{k+1}\right)_{\mathbb{T}} \cap\left(\cup \mathbb{T}^{\Pi_{\varepsilon_{1}}}\right), k \in \mathbb{Z}$, we get

$$
v(t) \leq v\left(t_{k}^{+}\right) e_{-p}\left(t, t_{k}\right)+\int_{t_{k}}^{t} e_{-p}(t, \sigma(s))\left(L\left\|v_{s}\right\|_{\varepsilon_{1}}+M(r)\right) \Delta s,
$$

thus we obtain

$$
\begin{aligned}
& v(t) \leq v\left(t_{k}^{-}\right) e_{-p}\left(t, t_{k}\right)+\int_{t_{k}}^{t} e_{-p}(t, \sigma(s))\left(L\left\|v_{s}\right\|_{\varepsilon_{1}}+M(r)\right) \Delta s+L r e_{-p}\left(t, t_{k}\right), \\
& v\left(t_{k+1}^{-}\right) \leq v\left(t_{k}^{+}\right) e_{-p}\left(t_{k+1}, t_{k}\right)+\int_{t_{k}}^{t_{k+1}} e_{-p}\left(t_{k+1}, \sigma(s)\right)\left(L\left\|v_{s}\right\|_{\varepsilon_{1}}+M(r)\right) \Delta s .
\end{aligned}
$$

Using (4.28) and (4.29), we can solve the differential inequality (4.27) and obtain

$$
\begin{aligned}
v(t+s) \leq & v\left(t_{0}+s\right) \prod_{t_{0}+s<t_{k}<t+s} e_{-p}\left(t+s, t_{0}+s\right) \\
& +\sum_{t_{0}+s<t_{k}<t+s}\left(\prod_{t_{k}<t_{j}<t+s} e_{-p}\left(t+s, t_{k}\right)\right) L r \\
& +\int_{t_{0}+s}^{t+s} \prod_{\theta<t_{k}<t+s} e_{-p}(t+s, \sigma(\theta))\left(L\left\|v_{\theta}\right\|_{\varepsilon_{1}}+M(r)\right) \Delta \theta
\end{aligned}
$$

for all $t \in \mathbb{T} \cap\left(\cup \mathbb{T}^{\Pi_{\varepsilon_{1}}}\right),-s \in(-\infty, 0]_{\Pi_{\varepsilon_{1}}}$, which implies that

$$
\begin{aligned}
\left\|v_{t}\right\|_{\varepsilon_{1}} \leq & \prod_{t_{0}+s<t_{k}<t+s} e_{-p}\left(t+s, t_{0}+s\right)\left\|v_{t_{0}}\right\|_{\varepsilon_{1}} \\
& +\frac{L}{p}\left(1-e_{-p}\left(t, t_{0}\right)\right)\left\|v_{t}\right\|_{\varepsilon_{1}}+\frac{M(r)}{p}\left(1-e_{-p}\left(t, t_{0}\right)\right)+\frac{L r}{1-e_{-p}(\theta, 0)} \\
\leq & \prod_{t_{0}+s<t_{k}<t+s} e_{-p}\left(t+s, t_{0}+s\right)\left\|v_{t_{0}}\right\|_{\varepsilon_{1}}+\frac{L}{p}\left\|v_{t}\right\|_{\varepsilon_{1}}+L r+\frac{M(r)}{p}
\end{aligned}
$$

and thus

$$
\left\|v_{t}\right\|_{\varepsilon_{1}} \leq \frac{p}{p-L} \prod_{t_{0}+s<t_{k}<t+s} e_{-p}\left(t+s, t_{0}+s\right)\left\|v_{t_{0}}\right\|_{\varepsilon_{1}}+\frac{\operatorname{Lrp}+M(r)}{p-L} \leq r .
$$

Consequently, $\left\|v_{t}\right\|_{\varepsilon_{1}} \leq r$ for all $t \in \mathbb{T} \cap\left(\cup \mathbb{T}^{\Pi_{\varepsilon_{1}}}\right)$. The estimate (4.22) follows from the same argument as in the proof of Theorem 4.3. The proof is complete.

Example 4.1 Let $\mathbb{T}$ be an almost periodic time scale with $\mathbb{Z} \subset \mathbb{T}$ and $c \in P C_{r d}\left(\mathbb{T}, \mathbb{R}^{+}\right)$. Consider the following $\varepsilon$-equivalent impulsive dynamic equation:

$$
\begin{cases}x^{\Delta}(t)=-\frac{c(t)}{1+c(t) \mu(t)} x(t)+\frac{\sin \sqrt{3} t+\cos t}{1+c(t) \mu(t)} x_{t}, & t \neq t_{k}=k, k \in \mathbb{Z}, \\ \Delta x(k)=\frac{k-1}{2 k} x(k), & t=t_{k}=k .\end{cases}
$$

Obviously,

$$
F\left(t, x, x_{t}\right)=-\frac{c(t)}{1+c(t) \mu(t)} x(t)+\frac{\sin \sqrt{3} t+\cos t}{1+c(t) \mu(t)} x_{t}, \quad I_{k}(x(k))=\frac{k-1}{2 k} x(k), \quad k \in \mathbb{Z} .
$$


One can check that (4.30) is equivalent to the following impulsive dynamic equation:

$$
\begin{cases}x^{\Delta}(t)=-c(t) x^{\sigma}(t)+(\sin \sqrt{3}+\cos t) x_{t}, & t \neq t_{k}=k, k \in \mathbb{Z}, \\ \Delta x(k)=\frac{k-1}{2 k} x(k), & t=t_{k}=k .\end{cases}
$$

Hence, by (iii) from Lemma 4.1 in this paper and using Lemma 4.2 from [25], for $t \in$ $\mathbb{T} \cap\left(\cup \mathbb{T}^{\Pi_{\varepsilon}}\right)$, we can calculate that

$$
\begin{aligned}
D_{+}|x(t)-y(t)|^{\Delta} & =|x(t)-y(t)|^{\Delta}=\left[x(t)-y(t), x^{\Delta}(t)-y^{\Delta}(t)\right]_{\mu} \\
& =\left[x(t)-y(t), F\left(t, x, x_{t}\right)-F\left(t, y, y_{t}\right)\right]_{\mu} \\
& \leq\left[x(t)-y(t),-\frac{c(t)}{1+c(t) \mu(t)}(x(t)-y(t))+\frac{\sin \sqrt{3} t+\cos t}{1+c(t) \mu(t)}\left(x_{t}-y_{t}\right)\right]_{\mu} \\
& \leq \operatorname{sign}\left(x^{\sigma}(t)-y^{\sigma}(t)\right)\left(x^{\Delta}(t)-y^{\Delta}(t)\right) \\
& =\operatorname{sign}\left(x^{\sigma}(t)-y^{\sigma}(t)\right)\left(-c(t)\left(x^{\sigma}(t)-y^{\sigma}(t)\right)+(\sin \sqrt{3} t+\cos t)\left(x_{t}-y_{t}\right)\right) \\
& =-c(t)\left|x^{\sigma}(t)-y^{\sigma}(t)\right|+|\sin \sqrt{3} t+\cos t|\left\|x_{t}-y_{t}\right\|_{\varepsilon} \\
& =-c(t)\left(\mu(t)|x(t)-y(t)|^{\Delta}+|x(t)-y(t)|\right)+|\sin \sqrt{3} t+\cos t|\left\|x_{t}-y_{t}\right\|_{\varepsilon} .
\end{aligned}
$$

Therefore, we can obtain

$$
D_{+}|x(t)-y(t)|^{\Delta} \leq-\frac{c(t)}{1+c(t) \mu(t)}|x(t)-y(t)|+\frac{|\sin \sqrt{3} t+\cos t|}{1+c(t) \mu(t)}\left\|x_{t}-y_{t}\right\|_{\varepsilon}
$$

Also, for $|x| \leq r$, we can easily obtain

$$
|F(t, 0, \phi)| \leq \frac{2 r}{1+c(t) \mu(t)} \leq \frac{2 r}{\inf _{t \in \mathbb{T} \cap\left(\cup \mathbb{T} \Pi_{\varepsilon}\right)} 1+c(t) \mu(t)} \leq 2 r+\ln \frac{3}{2}:=M(r)
$$

and

$$
|F(t, x, \phi)| \leq \frac{(2+c(t)) r}{1+c(t) \mu(t)} \leq \frac{\left(2+\sup _{t \in \mathbb{T} \cap\left(\cup \mathbb{T} \Pi_{\varepsilon}\right)} c(t)\right) r}{\inf _{t \in \mathbb{T} \cap\left(\cup \mathbb{T} \Pi_{\varepsilon}\right)} 1+c(t) \mu(t)}:=N(r) .
$$

Further, we can obtain

$$
\left|I_{k}(x)-I_{k}(y)\right| \leq \tilde{N}(r)|x-y|, \quad I_{k}(\mathbf{0})=\mathbf{0}, \quad \tilde{N}(r)=\frac{1}{2}+r
$$

and

$$
\tilde{h}=\inf \left(t_{k}-t_{k-1}\right)=1>\frac{\ln (1+\tilde{N}(r))}{M(r)}=\frac{\ln \left(\frac{3}{2}+r\right)}{2 r+\ln \frac{3}{2}}, \quad \forall r>0 .
$$

Thus we can take $L=\frac{2}{\inf _{t \in \mathbb{T} \cap\left(\cup \mathbb{I} \Pi_{\varepsilon}\right)} 1+c(t) \mu(t)}<2$ such that there exists

$$
p>\max \left\{2+\frac{1}{r} \ln \frac{3}{2}, 2, \frac{5}{2}+r\right\}>\max \left\{\frac{M(r)}{r}, L\right\}
$$


such that

$$
\frac{\tilde{N}(r)}{1-e_{-p}(\theta, 0)}+\frac{L}{p}<\frac{\frac{1}{2}+r}{1-e^{-p}}+\frac{L}{p}<\frac{\frac{1}{2}+r}{-p}+\frac{2}{p}<\frac{3}{2 p}<1 \quad \text { since } p>2 .
$$

In addition, we can obtain

$$
\frac{3(p-L)\left(1-e_{-p}(\theta, 0)\right)}{p\left(3-2 e_{-p}(\theta, 0)\right)}<\frac{3\left(1-e_{-p}(\theta, 0)\right)}{3-2 e_{-p}(\theta, 0)}<\frac{1-e_{-p}(\theta, 0)}{1-\frac{2}{3} e_{-p}(\theta, 0)}<1 .
$$

Therefore, all the conditions from Theorem 4.4 are satisfied, then (4.30) has a unique almost periodic solution $u(t)$ in $\left.C_{r}^{*}\right|_{\mathbb{T} \cap\left(\cup \mathbb{T} \Pi_{\varepsilon}\right)}$ for $0<r<1$.

\section{Conclusion and further discussion}

Since impulsive dynamic equations with 'slight vibration' can be established on almost periodic time scales and describe many natural phenomena precisely, we propose a new type of $\varepsilon$-equivalent impulsive dynamic equations on almost periodic time scales to reflect such a 'slight vibration', which will contribute to the theory of dynamic equations on time scales and practical applications in the real world. From this paper, one can observe that $\varepsilon$-equivalent impulsive dynamic equations are 'little fuzzy' in time variables, thus there is a theoretical and practical significance to obtain the existence of solutions for this type of dynamic equations.

To study the existence of solutions for $\varepsilon$-equivalent impulsive functional dynamic equations on almost periodic time scales, we established several theorems in the paper (see Sections 3 and 4) to obtain some new existence results for solutions. These results are also new when $\mathbb{T}=\mathbb{R}$ and $\mathbb{T}=\mathbb{Z}$. This is the first investigation for this new type of functional dynamic equations with 'slight vibration' and several new methods are provided. However, many problems remain to be studied. From Definition 2.5, we introduce the concept of $\Delta$-sub-derivatives on time scales, which means that (2.5) is the most general functional dynamic equations with 'arbitrary vibration' in time variables. In Theorem 4.2, we provide sufficient conditions to guarantee the existence of solutions for (4.3) with 'arbitrary vibration'. Nevertheless, since this type of dynamic equations has complicated $\Delta$ sub-derivatives.

Competing interests

The authors declare that they have no competing interests.

Authors' contributions

All authors contributed equally to the manuscript and typed, read and approved the final manuscript.

\section{Author details}

${ }^{1}$ Department of Mathematics, Yunnan University, Kunming, Yunnan 650091, People's Republic of China. ${ }^{2}$ Department of Mathematics, Texas A\&M University-Kingsville, 700 University Blvd., Kingsville, TX 78363-8202, USA. ${ }^{3}$ School of Mathematics, Statistics and Applied Mathematics, National University of Ireland, Galway, Ireland.

\section{Acknowledgements}

This work is supported by Tian Yuan Fund of NSFC (No. 11526181), Yunnan Province Education Department Scientific Research Fund Project of China (No. 2014Y008), and Yunnan Province Science and Technology Department Applied Basic Research Project of China (No. 2014FB102). 


\section{References}

1. Bohner, M, Peterson, A: Dynamic Equations on Time Scales. An Introduction with Applications. Birkhäuser, Boston (2001)

2. Agarwal, RP, Bohner, M: Basic calculus on time scales and some of its applications. Results Math. 35, 3-22 (1999)

3. Hilger, S: Ein Maßkettenkalkül mit Anwendung auf Zentrumsmannigfaltigkeiten. Ph.D. Thesis, Universität. Würzburg (1988). (in German)

4. Atici, FM, Eloe, PW, Kaymakcalan, B: The quasilinearization method for boundary value problems on time scales. J. Math. Appl. 276, 357-372 (2002)

5. Bohner, M, Wintz, N: The Kalman filter for linear systems on time scales. J. Math. Anal. Appl. 406, 419-436 (2013)

6. Akhmet, MU, Turan, M: The differential equations on time scales through impulsive differential equations. Nonlinear Anal. 65, 2043-2060 (2006)

7. Wang, C, Agarwal, RP: Changing-periodic time scales and decomposition theorems of time scales with applications to functions with local almost periodicity and automorphy. Adv. Differ. Equ. 2015, Article ID 296 (2015)

8. Stamov, GT, Stamova, IM: Almost periodic solutions for impulsive neural networks with delay. Appl. Math. Model. 31, 1263-1270 (2007)

9. Deysach, LG, Sell, GR: On the existence of almost periodic motions. Mich. Math. J. 12, 87-95 (1965)

10. Bochner, S: A new approach to almost periodicity. Proc. Natl. Acad. Sci. USA 48, 2039-2043 (1962)

11. Kato, S: Almost periodic solutions of functional differential equations with infinite delays. Funkc. Ekvacioj 38, 505-517 (1995)

12. Kato, S, Imai, M: On the existence of periodic and almost periodic solutions for nonlinear systems. Nonlinear Anal. TMA 24, 1183-1192 (1995)

13. Fink, AM: Almost Periodic Differential Equations. Springer, Berlin (1974)

14. Wang, C, Agarwal, RP: Relatively dense sets, corrected uniformly almost periodic functions on time scales, and generalizations. Adv. Differ. Equ. 2015, Article ID 312 (2015)

15. Wang, C, Agarwal, RP: A further study of almost periodic time scales with some notes and applications. Abstr. Appl. Anal. 2014, Article ID 267384 (2014)

16. Wang, C, Agarwal, RP: Almost periodic dynamics for impulsive delay neural networks of a general type on almost periodic time scales. Commun. Nonlinear Sci. Numer. Simul. 36, 238-251 (2016)

17. Wang, C, Agarwal, RP, O'Regan, D: Matrix measure on time scales and almost periodic analysis of the impulsive Lasota-Wazewska model with patch structure and forced perturbations. Math. Methods Appl. Sci. (2016). doi:10.1002/mma.3951

18. Wang, C, Agarwal, RP: A classification of time scales and analysis of the general delays on time scales with applications. Math. Methods Appl. Sci. 39, 1568-1590 (2016)

19. Morris, SA, Noussair, ES: The Schauder-Tychonoff fixed point theorem and applications. Math. Slovaca 25(2), 165-172 (1975)

20. Peterson, A, Thompson, B: Henstock-Kurzweil delta and nabla integrals. J. Math. Anal. Appl. 323, 162-178 (2006)

21. Zhu, ZQ, Wang, QR: Existence of nonoscillatory solutions to neutral dynamic equations on time scales. J. Math. Anal. Appl. 335, 751-762 (2007)

22. Fran̆ková, D: Regulated functions. Math. Bohem. 116, 20-59 (1991)

23. Mesquita, JG: Measure functional differential equations and impulsive functional dynamic equations on time scales. Doctoral dissertation. Instituto de Ciências Matemáticas e de Computação (2012)

24. Wang, C: Almost periodic solutions of impulsive BAM neural networks with variable delays on time scales. Commun. Nonlinear Sci. Numer. Simul. 19, 2828-2842 (2014)

25. Li, Y, Wang, C: Almost periodic solutions to dynamic equations on time scales and applications. J. Appl. Math. 2012 Article ID 463913 (2012)

\section{Submit your manuscript to a SpringerOpen ${ }^{\ominus}$ journal and benefit from:}

- Convenient online submission

Rigorous peer review

- Immediate publication on acceptance

Open access: articles freely available online

High visibility within the field

- Retaining the copyright to your article 